\title{
ZUSGS
}

science for a changing world

\section{REPORT FOR EXPLOSION AND EARTHQUAKE DATA ACQUIRED IN THE 1999 SEISMIC HAZARDS INVESTIGATION OF PUGET SOUND (SHIPS), WASHINGTON}

By Thomas M. Brocher ${ }^{1}$, Thomas L. Pratt², Kate C. Miller ${ }^{3}$, Anne M. Trehú, Catherine M. Snelson ${ }^{3}$, Craig S. Weaver, Ken C. Creager ${ }^{6}$, Robert S. Crosson ${ }^{6}$, Uri S. ten Brink ${ }^{7}$, Marcos G. Alvarez ${ }^{8}$, Steven H. Harder ${ }^{3}$, and Isa Asudeh ${ }^{9}$

Open-File Report 00-318

2000

This report is preliminary and has not been reviewed for conformity with U.S. Geological Survey editorial standards or with the North American Stratigraphic Code. Any use of trade, firm, or product names is for descriptive purposes only and does not imply endorsement by the U.S.

Government.

\section{U.S. DEPARTMENT OF THE INTERIOR U.S. GEOLOGICAL SURVEY}

${ }^{1}$ U.S. Geological Survey, 345 Middlefield Road, M/S 977, Menlo Park, CA 94025

2 U.S. Geological Survey, School of Oceanography, Box 357940, Univ. Washington, Seattle, WA 98195

${ }^{3}$ Dept. of Geol. Sci., Univ. Texas, El Paso, TX 79968

${ }^{4}$ College of Oceanic and Atmospheric Sci., Oregon State Univ., Corvallis, OR 97331

${ }^{5}$ U.S. Geological Survey, Geophysics, Box 351650, Univ. of Washington, Seattle, WA 98195

${ }^{6}$ Geophysics, Box 351650, Univ. of Washington, Seattle, WA 98195

${ }^{7}$ U.S. Geological Survey, Woods Hole, MA 02543

8 PASSCAL Instrument Center, New Mexico Tech, 801 Leroy Place, Socorro, NM 87801

${ }^{9}$ Geological Survey of Canada, 615 Booth St.., Ottawa, Ontario, K1A 0E9, Canada 


\section{CONTENTS}

$\begin{array}{lr}\text { Abstract } & \text { iii } \\ \text { Introduction } & 1 \\ \text { Data Acquisition } & 2 \\ \text { Experiment Design } & 2 \\ \text { Seismographs } & 2 \\ \text { Seismograph Deployment } & 4 \\ \text { Detonation of Shot Points } & 4 \\ \text { Earthquakes } & 5 \\ \text { Data Downloading } & 5 \\ \text { Station and Shot Point Locations } & 5 \\ \text { SEGY Data Merging } & 7 \\ \text { Station Numbers } & 7 \\ \text { SEGY Trace Format } & 8 \\ \text { Earthquakes } & 9 \\ \text { Data Quality } & 10 \\ \text { Data Availability } & 12 \\ \text { Acknowledgments } & 12 \\ \text { References Cited } & 13\end{array}$

\section{TABLES}

Table 1. Recording Parameters Used by the Five Different Types of Recorders 15

Table 2. Receiver station list (WGS 1984 Datum) 16

Table 3. Shot list ordered by chronologically by shot time 28

Table 4. Shot list ordered by geographic shotpoint location (west to east) 29

Table 5a. Shot points which triggered the Pacific Northwest Seismic Network (PNSN) 30

Table 5b. Position errors of Shots Located by the PNSN in Table 3a. 30

Table 6a. Earthquakes (and Blasts) in Western Washington, September 18 to 22, $1999 \quad 31$

Table 6b. Recorded Teleseisms, September 18 to 22, 1999

Table 7. SEGY trace header values used for Dry SHIPS SEGY Tapes 32

\section{FIGURES}

Figure 1. Map showing major sedimentary basins, cities, and locations of SHIPS seismic line and shot points $\quad 33$

$\begin{array}{lll}\text { Figure 2. Record section for SP1a } & 34\end{array}$

Figure 3. Record section for SP1b 235

Figure 4. Record section for SP2 36

$\begin{array}{lll}\text { Figure 5. Record section for SP4 } & 37\end{array}$

$\begin{array}{lll}\text { Figure 6. Record section for SP5a } & 38\end{array}$

$\begin{array}{ll}\text { Figure 7. Record section for SP5b } & 39\end{array}$

Figure 8. Record section for SP6 4

Figure 9. Record section for SP8 41

Figure 10. Record section for SP9 42

Figure 11. Record section for SP10 43

Figure 12. Record section for SP11a 44

Figure 13. Record section for SP11b 45

Figure 14. Record section for SP12a 46

Figure 15. Record section for SP12b 47

Figure 16. Record section for SP13 48

Figure 17. Record section for SP14 49 
Figure 18. Record section for SP15 50

Figure 19. Record section for SP17 51

Figure 20. Record section for SP18 $\quad 52$

Figure 21. Record section for SP19a

Figure 22. Record section for SP19b

Figure 23. Record section for SP20

Figure 24. Record section for SP21a

Figure 25. Record section for SP21b

Figure 26. Record section for SP22

Figure 27. Record section for SP24a $\quad 59$

Figure 28. Record section for SP24b $\quad 60$

Figure 29. Record section for SP26a 61

Figure 30. Record section for SP26b 62

Figure 31. Record section for SP27 63

Figure 32. Record section for SP29 64

Figure 33. Record section for SP30

Figure 34. Record section for SP31 66

Figure 35. Record section for SP32a

Figure 36. Record section for SP32b 68

Figure 37. Record section for SP33 69

Figure 38. Record section for SP34 70

Figure 39. Record section for SP35 71

Figure 40. Vertical component record of a local M2.8 earthquake 72

Figure 41. Horizontal component (N-S) record, low-pass filtered, of a local earthquake 73

Figure 42. Horizontal component (E-W) record, low-pass filtered, of a local earthquake 74

Figure 43. Vertical component record of the $\mathrm{M}_{\mathrm{w}}$ 7.6 Chi-Chi (Taiwan) earthquake 75

Figure 44. Vertical component record, low-pass filtered, of the $\mathrm{M}_{\mathrm{w}}$ 7.6 Chi-Chi

(Taiwan) earthquake

Figure 45. E-W horizontal component record of the Chi Chi (Taiwan) earthquake 77

Figure 46. N-S horizontal component record of the Chi Chi (Taiwan) earthquake 78

Figure 47. N-S horizontal component record, low-pass filtered, of the Chi Chi earthquake 79

Figure 48. Map showing stations that recorded the Chi Chi (Taiwan) earthquake 80

Figure 49. Relative shear wave amplitudes of the Chi Chi (Taiwan) earthquake 81 


\begin{abstract}
This report describes the acquisition, processing, and quality of seismic reflection and refraction data obtained in the Seattle basin, central Puget Lowland, western Washington, in September 1999 during the Seismic Hazards Investigation of Puget Sound (SHIPS). As a sequel to the 1998 SHIPS air gun experiment (also known as "Wet SHIPS"), the 1999 experiment, nicknamed "Dry SHIPS", acquired a 112-km-long east-west trending multichannel seismicreflection and refraction line in the Seattle basin. One thousand and eight seismographs were deployed at a nominal spacing of 100 meters and 29 shot points were detonated at approximately 4 $\mathrm{km}$ intervals along the seismic line. The wide-angle seismic profile was designed to (1) determine the E-W geometry of Seattle basin, (2) measure the seismic velocities within the basin, and (3) define the basement structure underlying the Seattle basin. In this report, we describe the acquisition of these data, discuss the processing and merging of the data into common shot gathers, and illustrate the acquired profiles. We also describe the format and content of the archival tapes containing the SEGY-formatted, common-shot gathers. Data quality is variable but useful data were acquired from all 29 shot points fired along the Dry SHIPS seismic line. The data show pronounced travel time delays associated with the low velocity sedimentary rocks filling the Seattle basin.

Thirty-five REFTEK stations, deployed at $4 \mathrm{~km}$ intervals along the Dry SHIPS line, recorded 26 regional earthquakes and blasts and 17 teleseismic events, including the main shock and several aftershocks of the $M_{w}=7.6$ Chi-Chi (Taiwan) earthquake of 9/20/1999. The teleseismic recordings of the Chi-Chi (Taiwan) mainshock provide useful signals down to 10 second periods. They document a significant (factor between 5 and 10) focusing of compressional- and shear-wave energy by the Seattle basin at periods between 1 and 2 seconds relative to "bedrock" sites east and west of the basin. Signal durations in the Seattle basin were also substantially increased relative to "bedrock" sites in the Olympic peninsula and Cascade foothills.
\end{abstract}




\section{INTRODUCTION}

In the past decade three major seismic hazards to western Washington and British Columbia have been recognized. Large (M 9) magnitude earthquakes along the Cascadia subduction zone megathrust have been proposed and documented in the geological record (Heaton and Kanamori, 1984; Atwater, 1987; Heaton and Hartzell, 1987; Hyndman et al., 1990; Hyndman and Wang, 1993; Atwater, 1996; Atwater and Hempill-Haley, 1997). Earthquakes within the subducting Juan de Fuca plate also represent significant seismic hazards (Weaver and Baker, 1988). Finally, crustal faults capable of large (M 7) magnitude earthquakes within the Puget Lowland have been inferred and mapped using a variety of methods including paleoseismicity, seismicity, seismic reflection, gravity and aeromagnetics (Atwater and Moore, 1992; Bucknam et al., 1992; Johnson et al., 1994, 1996, 1999; Pratt et al., 1997; Wells et al., 1998).

A March 1998 survey known as "Wet SHIPS (Seismic Hazards Investigation in Puget Sound)" performed a large scale investigation of the regional crustal structure of the Puget Lowland using airgun sources and land recorders during March 1998 (Brocher et al., 1999; Fisher et al., 1999). The purpose of Wet SHIPS was to obtain new, three-dimensional structural control on the seismogenic structures and Cenozoic basins in western Washington and southwestern British Columbia.

In this report we describe data obtained in September 1999 during a focused seismic investigation of the crustal structure in central Puget Sound, in an experiment nicknamed "Dry SHIPS”. During Dry SHIPS we recorded 38 shots using 1008 portable seismic stations at offsets up to $112 \mathrm{~km}$. A primary goal of Dry SHIPS was to provide compressional and shear wave velocity information for the sedimentary basin fill of the Seattle basin to allow better forecasts of strong ground motion focusing in the Seattle area (Figure 1). The new 3-D models developed from Dry SHIPS will be used to calculate synthetic seismograms to help understand the lateral variations of strong ground motions in the Seattle area. 


\section{DATA ACQUISITION}

Experiment Design

The Dry SHIPS seismic survey was designed to provide low-fold reflection coverage and high-fold refraction coverage along an east-west line through the center of the Seattle basin. The average shot spacing of $4 \mathrm{~km}$ was chosen to provide complete subsurface coverage beginning at about 2 seconds two-way travel time (above the base of the thickest part of the Seattle basin). The line orientation was selected to cross the thickest part of the Seattle basin (Finn et al., 1991) in an east-west direction. The line location was determined by the geometry of the local waterways and public lands. Because a true east-west line could not be fit through the study area, the line was broken into two line segments, lines 1 and 2, which overlap for $12 \mathrm{~km}$ on Kitsap Peninsula (Fig. 1). The overlap of the ends of lines 1 and 2 was designed to provide full-fold reflection coverage along the combined seismic line. Four shorter N-S trending fan lines, lines 3 to 6 , were designed to

provide three-dimensional control on the geometry of the eastern end of the Seattle basin. The nominal seismograph spacing along the fan lines was $1 \mathrm{~km}$ (Figure 1).

Station and shotpoint numbers increase from west to east (Figure 1). Line 1, $39 \mathrm{~km} \mathrm{long,}$ started near the eastern end of Olympic National Park at Station 1000 and ended at the eastern end of Kitsap Peninsula at Station 1390. Line 2 was $87.8 \mathrm{~km}$ long and started at the western end of Kitsap Peninsula at Station 2000 and ended to the east along the north fork of the Snoqualmie River in the western foothills of the Cascade Mountains at Station 2878. Line 3, $22 \mathrm{~km}$ long, trended north along Bainbridge Island (Stations 3000 to 3210). Line 4, $25 \mathrm{~km}$ long, trended north along the western shore of Lake Washington (Stations 4010 to 4250). Line 5, $22 \mathrm{~km}$ long, trended north along the western shore of Lake Sammamish (Stations 5000 to 5210). Line 6, $25 \mathrm{~km}$ long, trended north to the east of Lake Sammamish (Stations 6000 to 6240). Station numbers for Lines 3 to 6 increase from north to south (Figure 1).

\section{$\underline{\text { Seismographs }}$}

Five different types of portable seismographs were used during Dry SHIPS (Table 1). The 1008 recorders were deployed at a nominal station spacing of $100 \mathrm{~m}$, except for the ends of the lines, where a nominal station spacing of $200 \mathrm{~m}$ was used. The five types of recorders included: Texans (440 units), REFTEKs (231 units), Portable Refraction Seismographs (PRS-1's and PRS4's; 200 units), Seismic Group Recorders (SGR-III's; 129 units), and USGS Ocean bottom seismometers ("OBS's"; 8 units). The different types of land seismographs were interspersed uniformly along the line to provide a uniform instrument types along the line. Because the Texan units were completely buried, they were used in city parks and public areas to minimize vandalism 
or theft of the instruments. As a rule, the seismographs were programmed to record only 72 planned shot windows. Thirty-five REFTEKs, however, each having a 1-GByte hard-disk drive, were programmed to record continuously during their deployment to obtain records for local earthquakes and teleseisms occurring during Dry SHIPS. These 35 REFTEKs were deployed at about $4 \mathrm{~km}$ intervals along the seismic line and are annotated in Table 2. The Reftek model type, geophone type, and gains used for the continuously recorded REFTEKs were identical.

The Texans are single-component digital seismographs that record the signal from a single Mark Products® 1-10B vertical-component 4.5-Hz geophone. The main operational problems encountered with the Texans were (1) a shorter than anticipated battery life for some units and (2) a software glitch that resulted in the failure to download the complete recording program into 5 to $10 \%$ of the Texans.

The REFTEKs are described by PASSCAL (1991) and Brocher et al. (1999). For this experiment, the REFTEKs recorded signals from Mark Products® L-28 three-component 4.5-Hz geophones. The 3-component sensors were oriented with compasses such that the N-S component was directed to magnetic north. Almost all the REFTEKs were equipped with Global Positioning System (GPS) receivers to synchronize the internal timing on the individual REFTEKs to satellite timing. The main operational problem encountered during the deployment of the REFTEKs was a difficulty in obtaining a lock on the GPS satellites in forested areas.

The Portable Refraction Seismographs (PRS-1s and PRS-4s) used during Dry SHIPS have been described by Asudeh et al. (1992, 1998) and Luetgert et al. (1993). A single component from a Mark Products® L4A 2-Hz vertical component geophone was recorded. All 200 PRSs deployed yielded useful data apart from two that were stolen: one at Station 5100 was recovered but had been powered down causing the data from it to be lost. The other PRS stolen was deployed at Station 2143 and was never recovered.

The Seismic Group Recorders (SGR-III's) were described by Luetgert et al. (1993). The SGR-III's recorded the vertical component from Mark Products® L4A 2-Hz geophones. The 129 SGR's were programmed to record 24 different shot windows for each of the three nights of shooting. SGR tapes were changed after each night of shooting, yielding a set of three SGR tapes per seismograph. The main operational problem for the SGR's was a shortage of new batteries, requiring the reuse of old batteries. The use of old batteries and lack of maintenance of the SGR's contributed to many of the failures of the SGR's.

The 8 USGS OBS's used in Dry SHIPS were described by Brocher et al. (1999). All the OBS's (OBS1 through OBS8) were deployed in Puget Sound between Bainbridge Island and Seattle (Station 2190-2232; Figure 1, Table 2) and were programmed to record continuously. OBS locations were determined using differential GPS navigation and are believed to be accurate to within 10-20 meters. Depths were determined using available bathymetric maps and are believed to 
be accurate to within 10 meters. The OBS locations and water depths are provided in Table 4. Four channels were recorded by the OBS's, including three from a gimbaled, 3-component 4.5 Hz Mark Products ${ }^{\circledR}$ L15B seismometer and one from an OAS ${ }^{\circledR}$ E-2S hydrophone. OBS horizontal seismometer orientations were not recorded. All the OBS's were equipped with Seascan ${ }^{\circledR}$ clocks with accuracy of $1 \times 10^{-8} \mathrm{sec}^{-1}$ that have a clock drift of $\sim 1 \mathrm{msec}$ per day. A linear drift rate was assumed for the duration of the experiment and times were corrected accordingly.

\section{Seismograph Deployment}

The 231 Reftek recorders were deployed during a two-day period from Julian Day (JD) 261 to JD 262 (September $18^{\text {th }}$ to September 19th). The remaining 769 land seismographs were deployed on JD 262 (September $19^{\text {th }}$ ). All the land seismographs were retrieved on JD 265 $\left(\right.$ September $22^{\text {nd }}$ ). Only three instruments (two PRSs and one OBS) were lost or stolen, and only a few other seismographs were tampered with.

The OBS's were deployed on JD 258 (September 15) and were programmed to start recording continuous data at 0800 UTC on JD 260 (September 17). Recording by the OBS's ended upon recovery of the OBS's on JD 265 (September 22 ${ }^{\text {nd }}$ ). OBS D9 (Station 2190) used a $1 \mathrm{~Hz}$ geophone, and as expected, the signals for it are smaller than for the other OBS's, which used 4.5 $\mathrm{Hz}$ geophones.

\section{$\underline{\text { Detonation of Shot points }}$}

Shot hole loading began on September $9^{\text {th }}$ and was completed on September 21st, the last evening of shooting. The shots were detonated on three consecutive evenings, generally under still and warm conditions.

A total of 38 shots were detonated at 29 different shot points, numbered 1 to 35, from west to east (Fig. 1; Table 3). At nine shot points, shots were repeated to allow stacking of the shots to increase data quality. The shot sizes ranged from $25 \mathrm{lbs}(11.4 \mathrm{~kg})$ to $2500 \mathrm{lbs}(1136.4 \mathrm{~kg}) \mathrm{of}$ ammonium nitrate emulsion. The main charge was detonated using $1 \mathrm{lb}$ boosters ignited by Primacord $®$ detonating cord. The detonating cord was ignited by an electrical blasting cap using shot systems whose clocks were set to a GPS master clock accurate to within a millisecond. The clock drift of each shot system was measured to determine whether correction to the shot time was necessary. Table 3 presents this shotpoint data in the chronological order that the shots were fired. Latitudes and longitudes of the shot points are given in WGS 1984 datum and in UTM eastings and northings (Zone 10). Table 4 summarizes the shot information in geographical order from west to east, and, in addition, provides the name of the lead shooter for the shot. 
Several of the largest shots triggered the Pacific Northwest Seismic Network (PNSN). Table 5a identifies shots that triggered the PNSN. Hypocentral locations of the shots in Table 5a are those determined by the PNSN. Errors in these locations determined from their measured GPS locations are tabulated in Table 5b. The average error in latitude is $0.9 \mathrm{~km}$; the average error in longitude is $1.6 \mathrm{~km}$; the average total distance error in location is $2.0 \mathrm{~km}$; the average depth error is $2.1 \mathrm{~km}$. The errors are systematic in that they are smaller, on average, in the middle of the seismic line, between SP 17 and 22 (Bainbridge Island to Redmond), than on either end of the seismic line (Figure 1).

Earthquakes:

Thirty-five continuously recording REFTEK stations and the 8 OBS's, deployed at $4 \mathrm{~km}$ intervals along the Dry SHIPS line, recorded 26 local earthquakes and quarry blasts having magnitudes between -0.1 and 3.2 (Table $6 \mathrm{a}$ ). The REFTEK stations that recorded continuously are shown in Figure 48 (and marked with dots in the second column of Table 4). Events 3, 6, 11 to 13, and 21 to 22 occurred closest to the seismic line (Figure 48).

Seventeen teleseisms, including the mainshock of the $\mathrm{M}_{\mathrm{w}}$ 7.6 Chi-Chi (Taiwan) earthquake (Shin et al., 2000) (Event 4) and 10 of its aftershocks, were recorded during Dry SHIPS as determined by the USGS National Earthquake Information Center at Golden, Colorado (Table 6b).

\section{Data Downloading}

Data recorded by the Texans, REFTEK's, and PRS's were downloaded in the field at the Kitsap County Fairgrounds, Washington on the day of instrument pickup, JD 265 (September 22 ${ }^{\text {nd }}$, 1999). The OBS data were downloaded after the experiment (by October 27, 1999) at Woods Hole, Massachusetts. Data recorded on cassette tape by the SGR's were downloaded at Menlo Park, California on JD 361 (December 27nd, 1999) and were reduced there on January 5, 2000.

\section{$\underline{\text { Station and Shotpoint Locations: }}$}

Shotpoint and seismograph locations and elevations provided in Tables 2 and 3 are based on differential GPS measurements, using the World Geodetic System (WGS) 1984 datum. The roving GPS receiver occupied each station for about 2 minutes, yielding a nominal differential accuracy (standard deviation) of about 1 meter, in the horizontal. The nominal vertical accuracy of these determinations is lower, being about 2 meters (standard deviation), although this nominal accuracy seems unlikely and is demonstrably larger for many stations. The locations for 10 stations were not 
determined using differential GPS; for these stations the locations and elevations (WGS 1984 datum) were picked from digital USGS 7-1/2 minute topographic maps on a TOPO® CD-Rom. These stations are annotated on Table 2. 


\section{SEGY DATA MERGING}

\section{$\underline{\text { Station numbers }}$}

To avoid letters of the alphabet appearing in the shotpoint and receiver station names, all names were changed using numerals only. The shot points (SP1 - SP35) are multiplied by 10, with 1 added for an 'a' and 2 for a 'b'. Thus, shot point SP5a becomes shotpoint number 51 and SP5b becomes shot point number 52. The USGS OBS stations (OBS1 - OBS8) were numbered as Station 2190, 2196, 2202, etc. Only one land station, Station 1148a, had a letter in its name; it lies between 1148 and 1149 and is renumbered as Station 0148. Stations 1300, 1387, and 2283 are listed twice in Table 2: two different instruments were accidentally placed at each of these locations. Station 2057 is the location of the instrument center at Kitsap Fairgrounds (Figure 1). Station 5007 was an extra seismograph deployed at a participant's house located just east of the northern end of line 5.

\section{Instrument numbers}

The instrument numbers have been changed as follows

PRS Axxx are changed to PRS 1xxx (1000-1999); these are the PRS-1's

PRS Oxxx are changed to PRS 2xxx (2000-2999); these are the PRS-4's

OBS A3 changed to 3003 (etc. for all OBS's) (3001-3009)

\section{Conversion to UTM coordinates}

The WGS 1984 coordinates were converted to UTM zone 10 North coordinates in NAD83 using the National Geophysical Data Center's UTM algorithm UTMS (http://www.ngs.noaa.gov/PC_PROD/pc_prod.shtml).

\section{$\underline{\text { Reftek and Texan data processing }}$}

Clock drift correction: previously made during preprocessing of Reftek and Texan data Debias by subtracting the mean trace amplitude from every sample

Increase trace length to 62 seconds by adding 2 seconds of zero values to the beginning of the traces (recorded traces start at the shot time)

Put UTM geometry into SEGY headers

\section{PRS data processing}

Clock drift correction: static shift using values in headers

Debias by subtracting the mean trace amplitude from every sample 
Resample to $4 \mathrm{msec}$ sample interval from $8 \mathrm{msec}$ sample interval (125 samples/sec) using RESAMP in the seismic reflection processing software package SU

Increase trace length to 62 seconds from 57 seconds by adding 5 seconds of zero values to the end of the traces (recorded traces start 2 seconds prior to the shot time)

Put UTM geometry into SEGY headers

\section{SGR data processing}

Clock drift correction: previously made during preprocessing of SGR data

Debias by subtracting the mean trace amplitude from every sample

Resample to $4 \mathrm{msec}$ sample interval from $2 \mathrm{msec}$ sample interval (500 samples/sec) using

RESAMP in the seismic reflection processing software package SU

Increase trace length to 62 seconds from 31 seconds by adding 31 seconds of zero values to the end of the traces (recorded traces start 2 seconds prior to the shot time)

Put UTM geometry into SEGY headers

\section{OBS data processing}

Clock drift correction: previously made during preprocessing of OBS data

Resample to $4 \mathrm{msec}$ sample interval from $10 \mathrm{msec}$ sample interval (100 samples/sec) using

RESAMP in the seismic reflection processing software package SU

Shift start of trace to 2 seconds by adding 2 seconds of zero values to the beginning of the traces (recorded traces start at the shot time)

Truncate trace length to 62 seconds from 79 seconds

Debias by subtracting the mean trace amplitude from every sample

Put UTM geometry into SEGY headers

\section{SEGY Trace Format}

The merged common shot gathers generated by combining all of the data from the 5 types of seismographs were written in an unreduced travel-time format. Sixty-two seconds of data were saved for each trace, starting two seconds before the shot time. At a sample rate of $4 \mathrm{~ms}$, there are 15500 samples per trace, for a block length, including header, of 62240 bytes per trace.

SEGY trace header formats described by Barry et al. (1975) were modified slightly, as described in Table 7. Each merged record consists of a 240-byte header and a 62000 -byte data trace. All of the data trace values are written as 32 bit, IBM floating-point numbers (SEGY standard).

There are approximately 1400 total traces per common-shot gather. Of these, around 950 traces were recorded using vertical seismometers and the remaining 450 or so traces represent the horizontal geophone components recorded by the REFTEK's. 


\section{$\underline{\text { Earthquakes }}$}

The standard programs ref2segy, refrate, and segymerge were obtained from the PASSCAL Instrument Center and used to convert the REFTEK data to SEGY format, correct the clock drift, and make separate traces for these events (http://www.passcal.nmt.edu/software.shtml). (Events 17-19 represent three of our own shots.) These events were stored in SEGY format and archived to exabyte tape using unix tape-and-recovery (tar) commands. The local earthquakes and blasts archived to tape are listed in Table 6a.

These local earthquake data were archived in two exabyte tape formats. In the first format, the data are in PASSCAL segy format, generally with 600 seconds of data retained. On this archive tape there is a directory for each event, with each trace in a separate file named with the instrument and component of motion. The data values for each trace are preceded by a 240 byte header. The format of the header is given Table 7. All integer values are stored with the most significant byte first. Data values are 16 or 32 bit integers depending upon byte 206 of the header. Although there is a SEGY trace header for each trace, there are no IBM SEGY tape or binary headers.

In the second archival format for the local earthquakes, 120 seconds of unreduced SEGY data were saved for each event in the same format as the shot records (described below). The sample rate is $4 \mathrm{msec}$. The windows for this second format start either at the origin time, 15 seconds after the origin time, or 30 seconds after the origin time, increasing with epicentral distance. The data values for each trace are preceded by a 240 byte header that contains full geometry information in both latitude/longitude and UTM coordinates using the same header values as the shot records (described below).

Processing for the teleseismic events was similar to that used for the local events. The teleseism data were also archived in two exabyte tape formats. In the first tape format, the data are written in PASSCAL segy format with time windows from 10 minutes to 2 hours long (no geometry information has been placed into these headers). In the second tape format, the data were resampled to $80 \mathrm{msec}$ (12.5 samples/sec) and 42 minutes of unreduced data were saved for each event. In this second format, the headers contain the latitude and longitude of the source and receiver, the UTM coordinates for the receiver, but do not contain the source UTM coordinates or the source-receiver distance (offset). In the headers the sample rate is given as $8 \mathrm{msec}$ due to limitations in segy format for sample rate (a short integer limits the largest sample rate to 32.767 milliseconds). Thus the sample rate is a factor of ten too small, so that a 600 second trace (10 minutes) will appear to be 60 seconds long according to the header values. 


\section{DATA QUALITY}

The seismic reflection/refraction data recorded during SHIPS are plotted in Figures 2 to 39. Data quality is variable; we found large variations in shotpoint efficiency. Eleven of the larger shots that triggered the Pacific Northwest Seismic Network (PNSN) had network magnitudes between 1.0 and 2.7 (Table 3). Probably due to their location within the water table, shots in Seattle carried to much greater ranges than anticipated. In the following table, we briefly describe each shot.

\begin{tabular}{|c|c|c|}
\hline SP & $\begin{array}{r}\text { Shot } \\
\text { size } \\
(\text { lbs }) \\
\end{array}$ & Data Quality (A Qualitative Assessment) \\
\hline SP1 & 2800 & $\begin{array}{l}\text { SP1a yielded strong first arrivals that carried to the far eastern end of the line and provided } \\
\text { useful data along entire line through Seattle. SP1b, } 46 \mathrm{~m} \text { from SP1a, yielded much poorer } \\
\text { quality data that are traceable only about } 15 \mathrm{~km} \text { from the shot. }\end{array}$ \\
\hline $\mathrm{SP} 2$ & 250 & Weak shot that yielded faint first arrivals for $10 \mathrm{~km}$ \\
\hline SP4 & 250 & Shot yielded useful first arrivals for $20 \mathrm{~km}$. \\
\hline SP5 & 2000 & $\begin{array}{l}\text { Both SP5a and SP5b, } 169 \mathrm{~m} \text { apart, yielded high-amplitude first arrivals that carried the far } \\
\text { eastern end of line: arrivals recorded in Seattle are faint. }\end{array}$ \\
\hline SP6 & 50 & Fair shot that yielded first arrivals detected for $\pm 8 \mathrm{~km}$ on either side of the source. \\
\hline SP8 & 250 & Shot yielded useful data for $25 \mathrm{~km}$. \\
\hline SP9 & 50 & Shot yielded high-quality first arrivals for $25 \mathrm{~km}$. \\
\hline SP10 & 150 & Weak shot yielded observable first arrivals out to $\pm 7 \mathrm{~km}$. \\
\hline SP11 & 500 & $\begin{array}{l}\text { Both SP11a and SP11b, } 29 \text { m apart, were strong shots yielding arrivals can be followed to the } \\
\text { far eastern end of the line. Data recorded in Seattle are faint. Data recorded from SP11b are } \\
\text { slightly higher in quality than those recorded from SP11a. }\end{array}$ \\
\hline SP12 & 500 & $\begin{array}{l}\text { SP12a yielded poor arrivals. SP12b, } 30 \mathrm{~m} \text { from SP12a, yielded higher quality data observed for } \\
\pm 20 \mathrm{~km} \text { from the shotpoint. }\end{array}$ \\
\hline SP13 & 125 & Strong shot, yielded arrivals to nearly the eastern end of the line. \\
\hline SP14 & 50 & Fair shot, yielded arrivals for about $10 \mathrm{~km}$ from the shotpoint. \\
\hline SP15 & 50 & Strong shot, yielded useful arrivals as far as $30 \mathrm{~km}$ from the shotpoint. \\
\hline SP17 & 375 & Strong shot, yielded useful arrivals to both ends of the line and possibly traceable in Seattle. \\
\hline SP18 & 325 & Strong shot, yielded useful arrivals traceable through Seattle and out to both ends of the line. \\
\hline SP19 & 25 & Both SP19a and 19b, $18 \mathrm{~m}$ apart, yielded very faint arrivals, traceable only near the shotpoint. \\
\hline SP20 & 125 & $\begin{array}{l}\text { Strong shot, yielded useful arrivals out to both ends of the line. The arrivals have a lower } \\
\text { frequency character than most of the other shots along the line. }\end{array}$ \\
\hline SP21 & 400 & $\begin{array}{l}\text { Both SP21a and SP21b, } 45 \mathrm{~m} \text { apart, yielded large arrivals traceable through Seattle and out to } \\
\text { both ends of the line. }\end{array}$ \\
\hline SP22 & 400 & SP22 yielded large arrivals traceable throughout Seattle and out to the ends of the line. \\
\hline SP24 & 500 & Weak shots at both SP24a and SP24b, $30 \mathrm{~m}$ apart, because the main charge failed to detonate. \\
\hline
\end{tabular}




\begin{tabular}{|r|r|l|}
\hline & & Both shots yielded few useful arrivals. \\
\hline SP26 & 500 & $\begin{array}{l}\text { Both shots yielded comparable data quality. Both SP26a and SP26b, 46 m apart, produced } \\
\text { arrivals all along the eastern end of the line. }\end{array}$ \\
\hline SP27 & 250 & Shot yielded useful first arrivals for at least $10 \mathrm{~km}$ on both sides of the shotpoint. \\
\hline SP29 & 800 & Shot yielded useful first arrivals for $\pm 20 \mathrm{~km}$ of the shot. \\
\hline SP30 & 250 & $\begin{array}{l}\text { Strong shot yielded arrivals throughout the western end of the line. The arrivals in Seattle are } \\
\text { faint. }\end{array}$ \\
\hline SP31 & 250 & Weak shot yielded arrivals for $\pm 6 \mathrm{~km}$. \\
\hline SP32 & 2000 & $\begin{array}{l}\text { Both SP32a and SP32b, 30 m apart, yielded strong first arrivals seen to the far western end of } \\
\text { the line. Data are traceable through Seattle. }\end{array}$ \\
\hline SP33 & 250 & Strong shot yielded first arrivals detected for $15 \mathrm{~km}$. \\
\hline SP34 & 250 & Fair shot yielded first arrivals detected for $6 \mathrm{~km}$. \\
\hline SP35 & 2400 & $\begin{array}{l}\text { Strong shot yielded which large first arrivals that are traceable through Seattle and to the far } \\
\text { western end of the line. }\end{array}$ \\
\hline
\end{tabular}

Record sections for local earthquake 11 (Table 6a), a M2.8, 17-km deep event occurring near the eastern end of the line (Figure 48), are shown in Figures 40 to 42 . The vertical channel is aligned on the predicted P-wave arrival time (Figure 40; dotted line) with no filtering. The North component (Figure 41) and East component (Figure 42) are aligned on the predicted S-wave arrival time and have been low-pass filtered with a corner at $3 \mathrm{~Hz}$. Times are calculated for the iasp91 model that has $\mathrm{P}$ and $\mathrm{S}$ velocities of 5.8 and $3.36 \mathrm{~km} / \mathrm{s}$ above $20 \mathrm{~km}$ depth and 6.5 and $3.75 \mathrm{~km} / \mathrm{s}$ velocities below. Note the large travel time delays in both $\mathrm{P}$-wave and $\mathrm{S}$-wave arrivals in Seattle (Station 2262) associated with lower velocity sedimentary rocks in the Seattle basin.

Figures 43 to 47 present record sections showing 3-component recordings for the $M_{w}=7.6$ Chi-Chi (Taiwan) main shock of September 20, 1999 (Shin et al., 2000). In these figures, waveforms are aligned on the iasp91 predicted time for the P- and S-wave arrivals and are shifted using cross correlation for optimal alignment of the waveforms. The time shifts (relative traveltime residuals in seconds) are shown as the last numbers in the station labels. Labels show station number, epicentral distance (degrees), and azimuth (degrees). Traces are shown in true relative amplitude. In Figure 43, the P-wave arrivals have been low-pass filtered with a corner at $1 \mathrm{~Hz}$ (1 second period). Figure 44 shows the $\mathrm{P}$-wave record section that has been low pass filtered with a corner at $0.25 \mathrm{~Hz}$ (4 second period). Note the large (factor between 5 and 10) amplification of the signal at Station 2262, located in Seattle, in the middle of the Seattle basin, relative to Stations 1002 to 1082 in the Olympic Mountains west of Hood Canal and to Station 2768, in the Cascade foothills, outside of the Seattle basin (Figure 48). Similar results are seen in the shear wave arrivals, displayed in Figures 45 to 47 . Note that with the $89^{\circ}$ azimuth of propagation of these arrivals, the E-W horizontal component is nearly radial and the N-S horizontal component is nearly transverse to the direction of propagation. The locations of these recordings (Figure 48) reveal strong relative 
amplification of the P- and S-wave arrivals in the Seattle basin (Figure 49). The duration of large arrivals in the Seattle basin is also significantly longer than for stations located outside of the Seattle basin, approaching 100 seconds in the vicinity of Seattle (Figures 43-47).

\section{DATA AVAILABILITY}

Tape copies of the SEGY seismic data may be ordered via the World Wide Web from the IRIS/PASSCAL Data Management Center (DMC) in Seattle, Washington. The current Web site address of the Incorporated Research Institutions for Seismology (IRIS) Consortium is: http://www.iris.edu. The current general email address for the IRIS DMC is webmaster@iris.washington.edu.

\section{ACKNOWLEDGMENTS}

Tom Burdette, USGS, organized and arranged permits for the fieldwork. C. Bartlett, B. Laird, C. Tiballi, and A. Williams surveyed the line. K. Meagher and N. Sandoval coordinated the field logistics. J. Barnes, E. Barnett, K. Brown, J. Cox, D. Farrell, J. Murphy, R. Nicholson, B. Norris, K. Shedlock, R. Sliter, A. Williams and T. Yelin, all of the USGS, B. Hiett, G. Kaip, and K. Schramm, all of UTEP, B. Clement and L. Liberty, both from Boise State University, C. Bartlett, K. Kinports, J.D. Ousley, all of OSU, G. Bergsma, P. Burkholder, A. Cherkaoui, M. Edmunds, A. Lindemuth, A. Lisi, T. Qamar, K. Sauers, P. Shaw, C. Steedman, E. Sommargren, K. Tanno, G. Thomas, K. Troost, T. Van Wagoner, W. Wilcock, and R. Willis, all of the University of Washington (UW), and N. Ahmed, M. Benz, L. Clark, D. Cornwell, M. Conway, A. Lipede, G. Gandhok, J. Shragge, K. Smith, R. Smith, K. Walker, M. Bare, T. Bush, T. Butler, J. Cass, G. Civay, L. Cramer, D. Cysensky, G. Geehan, D. Hay, T. Hay, Lynn Hultgrien, T. Ise, P. Manganelli, P. Miller, D. Milne, J. Ramsey, P. Reed, C. Turnbull, H. Van Wandelen, J. Wilhite, and A. Winchester, helped to deploy and retrieve instruments. S. Azevedo, M. Fort, I. Matchum and W. Zamora, all of IRIS/PASSCAL, programmed and deployed the REFTEK's. J. Meloche and M. Gervay prepared the PRS's for deployment and helped deploy them. H. Benz, T. Burdette, E. Criley, D. Croker, S. Harder, R. Kaderabek, D. Reneau, and J. Van Schaack detonated the shotholes. T. Qamar and students performed the GPS survey of SP21. L. Preston, UW, processed the Reftek earthquake data and plotted Figures 43-49.

We thank the Washington State Departments of Forestry and Parks and Recreation, Olympic National Forest, the Bureau of Land Management, Kitsap and King Counties, and the Cities of Bainbridge Island, Seattle, and Redmond for permission to access land under their jurisdiction. We thank the Weyerhaeuser Corp., International Paper Co., and numerous smaller property owners for permission to access their land.

We thank NOAA-PMEL for the use of their vessel and facilities for staging, deployment, and recovery of the USGS OBSs, Captain N. Delich for his professional operation of the SP Hayes, and G. Miller, J. Nealon, and J. Newel for their help during OBS field operations.

Gary Fuis, USGS, critically reviewed this report.

This work was supported by the USGS Urban Geological Hazards Initiative, and external grants from the USGS National Earthquake Hazards Reduction Program to Oregon State University, the University of Texas El Paso, and the University of Washington. 


\section{REFERENCES CITED}

Asudeh I., F. Andersen, J. Parmelee, S. Vishnubhatla, P. Munro and J. Thomas, 1992, A Portable Refraction Seismograph PRS1, Geological Survey of Canada Open File Report No. 2478, Lithoprobe Publication No. 294, 34 pages.

Asudeh, I., D. White, B. Roberts, D. Forsyth, I. Kay, T. Cartwright, P. Carroll, Z. Hajnal, B. Koperwhats, G. Musacchio and D. Farrell, 1998, 1996 LITHOPROBE Western Superior Seismic Refraction Survey: Field Acquisition and Processing Report. Geological Survey of Canada Open File Report No. 3583, Lithoprobe Report No. 916, 223 pages.

Atwater, B.F., 1987, Evidence for great Holocene earthquakes along the outer coast of Washington State, Science, 236, 942-944.

Atwater, B.F., 1996, Coastal evidence for great earthquakes in western Washington, U.S. Geological Survey, Professional Paper 1560, p. 77-90.

Atwater, B.F., and Moore, A.L., 1992, A tsunami about 1000 years ago in Puget Sound, Washington, Science, v. 258, p. 1614-1617.

Atwater, B.F., and Hemphill-Haley, E., 1997, Recurrence intervals for great earthquakes of the past 3,500 years at northeastern Willapa Bay, Washington: U.S. Geological Survey Professional Paper 1576, 108 p.

Barry, K.M., D.A. Cravers, and C.W. Kneale, 1975, Recommended standards for digital tape formats: Geophysics, v. 40, p. 344-352.

Brocher, T.M., T. Parsons, K.C. Creager, R.S. Crosson, N.P. Symons, G.D. Spence, B.C. Zelt, P.T.C. Hammer, R. D. Hyndman, D.C. Mosher, A.M. Tréhu, K.C. Miller, U.S. ten Brink, M.A. Fisher, T.L. Pratt, M.G. Alvarez, B.C. Beaudoin, K.E. Louden, and C.S. Weaver, Wide-angle seismic recordings from the 1998 Seismic Hazards Investigation of Puget Sound (SHIPS), western Washington and British Columbia, U.S. Geological Survey Open-file Report 99-314, $110 \mathrm{pp}, 1999$.

Bucknam, R.C., Hemphill-Haley, E., and Leopold, E.B., 1992, Abrupt uplift within the past 1700 years at southern Puget Sound, Washington, Science, v. 258, p. 1611-1614.

Finn, C.A., W.M. Phillips, and D.L. Williams, Gravity anomaly and terrain maps of Washington, U.S. Geological Survey Geophysical Investigations Map GP-988, 1991.

Fisher, M.A., T.M. Brocher, R.D. Hyndman, A.M. Trehu, C.S. Weaver, K.C. Creager, R.S. Crosson, T. Parsons, A.K. Cooper, D. Mosher, G. Spence, B.C. Zelt, P.T. Hammer, U. ten Brink, T.L. Pratt, K.C. Miller, J.R. Childs, G.R. Cochrane, S. Chopra, and R. Walia, 1999, Seismic survey probes urban earthquake hazards in Pacific Northwest, EOS, Trans. Amer. Geophys. Un., v. 80, no. 2, p. 13-17.

Heaton, T.H., and H. Kanamori, 1984, Seismic potential associated with subduction in the northwestern United States: Seismological Society of America Bulletin, v. 74, p. 933-941.

Heaton, T.H., and S.H. Hartzell, 1987, Earthquake hazards on the Cascadia subduction zone, Science, v. 236, p. 162-168.

Hyndman, R.D., and K. Wang, 1993, Thermal constraints of major thrust earthquake failure: The Cascadia subduction zone: Journal of Geophysical Research, v. 98, p. 2039-2060.

Hyndman, R.D., Yorath, C.J., Clowes, R.M., and Davis, E.E., 1990, The northern Cascadia subduction zone at Vancouver Island: Seismic structure and tectonic history, Can. J. Earth Sci., 27, p. 313-329.

Johnson, S.Y., Potter, C.J., and Armentrout, J.M., 1994, Origin and evolution of the Seattle fault and Seattle basin, Washington, Geology, v. 22, p. 71-74.

Johnson, S.Y., Potter, C.J., Armentrout, J.M., Miller, J.J., Finn, C., and Weaver, C.S., 1996, The southern Whidbey Island fault, western Washington-An active structure in the Puget Lowland, Washington: Geological Society of America Bulletin, v. 108, p. 334-354, and oversized insert. 
Johnson, S.Y., S.V. Dadisman, J.R. Childs, W.D. Stanley, 1999, Active tectonics of the Seattle fault and central Puget Sound, Washington-Implications for earthquake hazards, Geological Society of America, Bulletin v. 111, p. 1042-1053.

Luetgert, J., W. Mooney, A. Trehu, J. Nabelek, G.R. Keller, K. Miller, I. Asudeh, and R. Isbell, 1993, Data report for a seismic refraction/wide-angle reflection investigation of the Puget basin and Willamette Valley in western Washington and Oregon, U.S. Geological Survey, Open-file Report 93-347, 45 pp. With 2 appendices.

PASSCAL, 1991, Users Guide, A Guide to Planning Experiments Using PASSCAL Instruments: IRIS, $28 \mathrm{pp}$.

Pratt, T.L., S. Johnson, C. Potter, W. Stephenson, and C. Finn, 1997, Seismic reflection images beneath Puget Sound, western Washington state: The Puget Lowland thrust sheet hypothesis, J. Geophys. Res., 102, 27,469-27,489.

Shin, T.C., K.W. Kuo, W.H.K. Lee, T.L. Teng, and Y.B. Tsai, 2000, A preliminary report on the 1999 Chi-Chi (Taiwan) earthquake, Seismological Res. Lett., v. 71, p. 24-30.

Weaver, C.S., and Baker, G.E., 1988, Geometry of the Juan de Fuca plate beneath Washington and northern Oregon from seismicity, Bulletin of the Seismological Society of America, v. 78, p. 264-275.

Wells, R.E., Weaver, C.S., and Blakely, R.J., 1998, Fore-arc migration in Cascadia and its neotectonic significance, Geology, 26, 759-762. 
Table 1. Recording Parameters Used by the Five Different Types of Seismographs

\begin{tabular}{lccccccc}
\hline $\begin{array}{c}\text { Instrument } \\
\text { Type }\end{array}$ & $\begin{array}{c}\text { Number } \\
\text { of } \\
\text { Units }\end{array}$ & $\begin{array}{c}\text { Record } \\
\text { Length } \\
\text { (seconds) }\end{array}$ & $\begin{array}{c}\text { Recording Start Time } \\
\text { (seconds before } \\
\text { shottime) }\end{array}$ & $\begin{array}{c}\text { Sample } \\
\text { Rate } \\
(\mathrm{Hz})\end{array}$ & $\begin{array}{c}\text { No. } \\
\text { of Geophone } \\
\text { Components }\end{array}$ & $\begin{array}{c}\text { Natural } \\
\text { Frequency } \\
\text { Geophone (Hz) }\end{array}$ & $\begin{array}{c}\text { Internal } \\
\text { Timing }\end{array}$ \\
\hline Reftek 06, 07 & 231 & 62 & 2 & & & & \\
PRS-1, PRS-4 & 200 & 58 & 2 & 250 & 3 & 4.5 & GPS \\
Texan & 440 & 60 & 0 & 125 & 1 (Vertical) & 2 & Pulsed \\
SGR-III & 129 & 31 & 2 & 500 & 1 (Vertical) & 4.5 & Pulsed \\
OBS & 8 & Continuous & Continuous & 100 & 3 & 4.5 & Pulsed \\
\hline
\end{tabular}

Two different types of REFTEKs were deployed; REFTEK Model 06's and 07's. REFTEK Models 06s (DAS No. 6000-6999) are 16-bit, 3-component recorders. REFTEK Models 07s (DAS No. 7000-7999) are 24-bit, 3-component recorders. The two different models of REFTEKs recorded channels 1 to 3 . Channel 1 was used for the vertical geophone component, channel 2 was used for the N-S oriented horizontal geophone component, and channel 3 was used for the E-W oriented horizontal geophone component. Eighty-five REFTEK Model 06's and 146 REFTEK Model 07's were deployed.

One hundred seventy-three PRS-I's and 27 PRS-4's were deployed. Only the vertical geophone component was used for the 3-component PRS-4 recorders.

Internal timing of the seismographs was synchronized to Universal Time either by using an internal GPS receiver to continuously record UTC (for the REFTEKS) or by setting the internal time from a master clock at the time of deployment and using this master clock to note the clock drift at the time that the receiver was retrieved (pulsed). 
Table 2. Receiver Station List (WGS 1984 Datum).

\begin{tabular}{|c|c|c|c|c|c|c|}
\hline$\underline{\text { Stake }}$ & $\frac{\text { Unit }}{\underline{\text { No. }}}$ & $\underline{\text { Latitude }}$ & Longitude & $\begin{array}{c}\underline{\mathrm{UTM}} \\
\underline{\text { Easting }}\end{array}$ & $\begin{array}{c}\text { UTM } \\
\text { Northing }\end{array}$ & $\frac{\text { Elev }}{\underline{\mathrm{m}}}$ \\
\hline 1000 & $* 501$ & 47.729631 & -123.135968 & 489805 & 5286259 & 435 \\
\hline 1002 & $\bullet 7283$ & 47.730413 & -123.133018 & 490026 & 5286346 & 411 \\
\hline 1004 & A066 & 47.730737 & -123.130403 & 490222 & 5286382 & 383 \\
\hline 1006 & 38 & 47.730517 & -123.127582 & 490434 & 5286357 & 364 \\
\hline 1008 & 6096 & 47.730625 & -123.124675 & 490651 & 5286369 & 359 \\
\hline 1010 & $* 503$ & 47.730128 & -123.122729 & 490797 & 5286313 & 368 \\
\hline 1012 & $* 504$ & 47.730119 & -123.119628 & 491030 & 5286312 & 346 \\
\hline 1014 & $* 505$ & 47.729801 & -123.118080 & 491146 & 5286276 & 329 \\
\hline 1016 & 6118 & 47.729201 & -123.113740 & 491471 & 5286209 & 347 \\
\hline 1018 & 46 & 47.728836 & -123.111405 & 491646 & 5286168 & 308 \\
\hline$\diamond 1020$ & $* 506$ & 47.728850 & -123.109020 & 491825 & 5286169 & 336 \\
\hline$\bullet 1022$ & 43 & 47.728780 & -123.106330 & 492027 & 5286161 & 350 \\
\hline • 1024 & 6038 & 47.728780 & -123.103920 & 492207 & 5286161 & 324 \\
\hline$\bullet 1026$ & A065 & 47.729000 & -123.101340 & 492401 & 5286185 & 314 \\
\hline • 1028 & 40 & 47.729270 & -123.098260 & 492632 & 5286215 & 301 \\
\hline$\bullet 1030$ & 6061 & 47.730410 & -123.095180 & 492863 & 5286342 & 250 \\
\hline • 1032 & $* 508$ & 47.731210 & -123.092320 & 493078 & 5286430 & 226 \\
\hline 1034 & 41 & 47.730944 & -123.089770 & 493269 & 5286400 & 218 \\
\hline 1036 & A067 & 47.734213 & -123.085712 & 493573 & 5286763 & 202 \\
\hline 1038 & $\bullet 7443$ & 47.735803 & -123.082747 & 493796 & 5286940 & 232 \\
\hline 1040 & 37 & 47.736645 & -123.080211 & 493986 & 5287033 & 229 \\
\hline 1042 & $* 509$ & 47.737734 & -123.076317 & 494278 & 5287154 & 203 \\
\hline 1044 & A068 & 47.738925 & -123.073318 & 494503 & 5287286 & 174 \\
\hline 1046 & 6128 & 47.739471 & -123.070344 & 494726 & 5287347 & 225 \\
\hline 1048 & A069 & 47.740054 & -123.067513 & 494939 & 5287411 & 198 \\
\hline 1050 & 36 & 47.740320 & -123.064485 & 495166 & 5287441 & 196 \\
\hline 1052 & $* 510$ & 47.740858 & -123.061857 & 495363 & 5287500 & 175 \\
\hline 1054 & 6047 & 47.741457 & -123.058983 & 495578 & 5287567 & 212 \\
\hline 1056 & 35 & 47.741650 & -123.056059 & 495797 & 5287588 & 171 \\
\hline 1058 & A070 & 47.741715 & -123.054349 & 495926 & 5287595 & 176 \\
\hline 1060 & 34 & 47.742000 & -123.051776 & 496119 & 5287627 & 161 \\
\hline 1062 & 6071 & 47.741646 & -123.049106 & 496319 & 5287587 & 144 \\
\hline 1064 & $* 511$ & 47.741347 & -123.046546 & 496511 & 5287554 & 156 \\
\hline 1066 & 33 & 47.741225 & -123.043788 & 496717 & 5287540 & 142 \\
\hline 1068 & A071 & 47.741412 & -123.040554 & 496960 & 5287561 & 142 \\
\hline 1070 & 6019 & 47.740922 & -123.038002 & 497151 & 5287506 & 140 \\
\hline 1072 & 30 & 47.740086 & -123.035594 & 497332 & 5287413 & 137 \\
\hline 1074 & $* 512$ & 47.739453 & -123.033163 & 497514 & 5287343 & 148 \\
\hline
\end{tabular}

\begin{tabular}{|c|c|c|c|c|c|}
\hline 1076 & 32 & 47.739184 & -123.028264 & 497881 & 5287313 \\
\hline 1078 & 6062 & 47.737804 & -123.026426 & 498019 & 5287159 \\
\hline 1080 & A072 & 47.736077 & -123.024298 & 498178 & 5286967 \\
\hline 1082 & $\bullet 7613$ & 47.735042 & -123.022541 & 498310 & 5286852 \\
\hline 1084 & $* 513$ & 47.733350 & -123.020359 & 498474 & 5286664 \\
\hline 1086 & 6109 & 47.731373 & -123.018147 & 498639 & 5286444 \\
\hline 1088 & A023 & 47.729293 & -123.016684 & 498749 & 5286213 \\
\hline 1090 & 6003 & 47.727969 & -123.014414 & 498919 & 5286066 \\
\hline 1092 & A025 & 47.727476 & -123.012544 & 499059 & 5286011 \\
\hline 1094 & 6080 & 47.725689 & -123.010093 & 499243 & 5285812 \\
\hline 1096 & $* 635$ & 47.725811 & -123.007254 & 499456 & 5285826 \\
\hline 1098 & 6125 & 47.725306 & -123.004269 & 499680 & 5285770 \\
\hline 1100 & A026 & 47.724611 & -123.002509 & 499812 & 5285693 \\
\hline 1101 & 6056 & 47.724208 & -123.001318 & 499901 & 5285648 \\
\hline 1102 & 31 & 47.723773 & -123.000205 & 499985 & 5285599 \\
\hline 1102 & 6110 & 47.723773 & -123.000205 & 499985 & 5285599 \\
\hline 1103 & $* 515$ & 47.723268 & -122.999018 & 500074 & 5285543 \\
\hline 1104 & A027 & 47.722828 & -122.997814 & 500164 & 5285494 \\
\hline 1105 & 6100 & 47.722433 & -122.996036 & 500297 & 5285451 \\
\hline 1106 & A028 & 47.721720 & -122.994475 & 500414 & 5285371 \\
\hline 1107 & A029 & 47.721618 & -122.993066 & 500520 & 5285360 \\
\hline 1108 & $* 659$ & 47.721477 & -122.991750 & 500619 & 5285344 \\
\hline 1109 & 6055 & 47.721320 & -122.990409 & 500719 & 5285327 \\
\hline 1110 & 27 & 47.720978 & -122.989183 & 500811 & 5285289 \\
\hline 1111 & A056 & 47.720748 & -122.987720 & 500921 & 5285263 \\
\hline 1112 & $* 518$ & 47.720241 & -122.986688 & 500998 & 5285207 \\
\hline 1113 & 6119 & 47.720155 & -122.985359 & 501098 & 5285197 \\
\hline 1114 & $* 520$ & 47.720170 & -122.984000 & 501200 & 5285199 \\
\hline 1115 & 26 & 47.720425 & -122.982724 & 501296 & 5285228 \\
\hline 1116 & A057 & 47.720646 & -122.981338 & 501400 & 5285252 \\
\hline 1117 & 6052 & 47.720705 & -122.979817 & 501514 & 5285259 \\
\hline 1118 & 29 & 47.720484 & -122.978471 & 501615 & 5285234 \\
\hline 1119 & $* 521$ & 47.720235 & -122.977161 & 501713 & 5285207 \\
\hline 1120 & 28 & 47.720008 & -122.975799 & 501815 & 5285181 \\
\hline 1121 & 6099 & 47.719939 & -122.974499 & 501913 & 5285174 \\
\hline 1122 & A058 & 47.719887 & -122.973288 & 502003 & 5285168 \\
\hline 1123 & $* 522$ & 47.719560 & -122.971951 & 502104 & 5285132 \\
\hline 1124 & 25 & 47.719168 & -122.970438 & 502217 & 5285088 \\
\hline 1125 & 6025 & 47.719315 & -122.969120 & 502316 & 5285104 \\
\hline 1126 & 643 & 47.719304 & -122.967687 & 502423 & 5285103 \\
\hline 1127 & A059 & 47.719422 & -122.966352 & 502524 & 5285116 \\
\hline 1128 & $* 523$ & 47.719393 & -122.965027 & 502623 & 5285113 \\
\hline 1129 & $\bullet 7593$ & 47.719386 & -122.963720 & 502721 & 5285113 \\
\hline 1130 & 73 & 47.719459 & -122.962370 & 502822 & 5285121 \\
\hline 1131 & 642 & 47.719513 & -122.961034 & 502922 & 5285127 \\
\hline
\end{tabular}




\begin{tabular}{|c|c|c|c|c|c|c|c|c|c|c|c|c|}
\hline 1132 & A060 & 47.719333 & -122.959456 & 503041 & 5285107 & 70 & 1176 & $* 536$ & 47.697426 & -122.906123 & 507044 & 5282676 \\
\hline 1133 & 6035 & 47.719266 & -122.958270 & 503130 & 5285099 & 55 & 1177 & A047 & 47.697548 & -122.904800 & 507143 & 5282689 \\
\hline 1134 & A061 & 47.719249 & -122.956941 & 503229 & 5285098 & 57 & 1178 & A046 & 47.697756 & -122.903430 & 507246 & 5282712 \\
\hline 1135 & $* 524$ & 47.719194 & -122.955566 & 503333 & 5285092 & 57 & 1179 & 64 & 47.697706 & -122.902157 & 507341 & 5282707 \\
\hline 1136 & A062 & 47.719239 & -122.954259 & 503431 & 5285097 & 50 & 1180 & 63 & 47.697588 & -122.900821 & 507441 & 5282694 \\
\hline 1137 & 6092 & 47.719285 & -122.952892 & 503533 & 5285102 & 55 & 1181 & A045 & 47.697490 & -122.899421 & 507546 & 5282683 \\
\hline 1138 & A063 & 47.719180 & -122.951557 & 503633 & 5285090 & 52 & 1182 & 62 & 47.697906 & -122.898015 & 507652 & 5282730 \\
\hline 1139 & 72 & 47.719035 & -122.950269 & 503730 & 5285074 & 58 & 1183 & $* 507$ & 47.698414 & -122.896314 & 507779 & 5282786 \\
\hline 1140 & $* 525$ & 47.718689 & -122.948936 & 503830 & 5285036 & 60 & 1233 & 59 & 47.693356 & -122.829128 & 512822 & 5282233 \\
\hline 1141 & 6034 & 47.718707 & -122.947605 & 503930 & 5285038 & 47 & 1234 & 57 & 47.692614 & -122.828646 & 512858 & 5282151 \\
\hline 1142 & 74 & 47.718835 & -122.946165 & 504038 & 5285052 & 46 & 1235 & 51 & 47.692462 & -122.827438 & 512949 & 5282134 \\
\hline 1143 & A064 & 47.718827 & -122.944910 & 504132 & 5285051 & 48 & 1236 & 54 & 47.692575 & -122.826048 & 513053 & 5282147 \\
\hline 1144 & $* 526$ & 47.718854 & -122.943645 & 504227 & 5285054 & 46 & 1237 & 6095 & 47.692792 & -122.824795 & 513147 & 5282171 \\
\hline 1145 & 6125 & 47.718446 & -122.942270 & 504330 & 5285009 & 44 & 1238 & A189 & 47.692401 & -122.823661 & 513232 & 5282128 \\
\hline 1146 & $* 527$ & 47.717754 & -122.941314 & 504402 & 5284932 & 47 & 1239 & 53 & 47.691973 & -122.822665 & 513307 & 5282080 \\
\hline 1147 & 70 & 47.715987 & -122.940348 & 504474 & 5284736 & 56 & 1240 & $* 537$ & 47.691487 & -122.820555 & 513465 & 5282027 \\
\hline 1148 & A030 & 47.713573 & -122.939833 & 504513 & 5284468 & 83 & 1241 & 6027 & 47.690660 & -122.820162 & 513495 & 5281935 \\
\hline • 0148 & 6065 & 47.712817 & -122.939354 & 504549 & 5284384 & 82 & 1242 & 56 & 47.689353 & -122.818594 & 513613 & 5281790 \\
\hline 1149 & 6020 & 47.712062 & -122.938875 & 504585 & 5284300 & 81 & 1243 & A 125 & 47.689166 & -122.817657 & 513683 & 5281769 \\
\hline 1150 & $* 528$ & 47.711711 & -122.937468 & 504691 & 5284261 & 81 & 1244 & 6081 & 47.689273 & -122.816540 & 513767 & 5281781 \\
\hline 1151 & 69 & 47.711681 & -122.936206 & 504785 & 5284258 & 97 & 1245 & 58 & 47.689407 & -122.815286 & 513861 & 5281797 \\
\hline 1152 & A050 & 47.710785 & -122.935185 & 504862 & 5284158 & 71 & 1246 & 52 & 47.689389 & -122.813945 & 513962 & 5281795 \\
\hline 1153 & 6050 & 47.710346 & -122.933889 & 504959 & 5284109 & 67 & 1247 & 60 & 47.689456 & -122.812374 & 514080 & 5281803 \\
\hline 1154 & 68 & 47.709547 & -122.932708 & 505048 & 5284021 & 56 & 1248 & 55 & 47.689923 & -122.810569 & 514215 & 5281855 \\
\hline 1155 & $* 529$ & 47.708758 & -122.931535 & 505136 & 5283933 & 59 & 1249 & A126 & 47.691198 & -122.808154 & 514396 & 5281997 \\
\hline 1156 & A049 & 47.708032 & -122.930351 & 505225 & 5283852 & 47 & 1250 & $* 538$ & 47.690385 & -122.807048 & 514479 & 5281907 \\
\hline 1157 & 6029 & 47.707381 & -122.929248 & 505308 & 5283780 & 40 & 1251 & $\bullet 7609$ & 47.690417 & -122.805850 & 514569 & 5281911 \\
\hline 1158 & A051 & 47.707031 & -122.928002 & 505401 & 5283741 & 41 & 1252 & 61 & 47.690420 & -122.804494 & 514671 & 5281911 \\
\hline 1159 & 67 & 47.706766 & -122.926726 & 505497 & 5283712 & 40 & 1253 & A127 & 47.693527 & -122.802200 & 514842 & 5282257 \\
\hline 1160 & $* 531$ & 47.706449 & -122.925415 & 505595 & 5283677 & 44 & 1254 & 50 & 47.695627 & -122.800300 & 514984 & 5282491 \\
\hline 1161 & 6101 & 47.705991 & -122.924179 & 505688 & 5283626 & 42 & 1255 & 6113 & 47.695567 & -122.798995 & 515082 & 5282484 \\
\hline 1162 & A031 & 47.705567 & -122.923024 & 505775 & 5283579 & 42 & 1256 & $* 539$ & 47.695594 & -122.797358 & 515205 & 5282488 \\
\hline 1163 & A033 & 47.705129 & -122.921855 & 505862 & 5283530 & 50 & 1257 & 48 & 47.695602 & -122.795983 & 515308 & 5282489 \\
\hline 1164 & $* 532$ & 47.704825 & -122.920605 & 505956 & 5283497 & 66 & 1258 & 6129 & 47.695581 & -122.794790 & 515398 & 5282487 \\
\hline 1165 & 6057 & 47.704641 & -122.919006 & 506076 & 5283476 & 70 & 1259 & $* 540$ & 47.695587 & -122.793195 & 515517 & 5282488 \\
\hline 1166 & 653 & 47.704455 & -122.917548 & 506186 & 5283456 & 72 & 1260 & 45 & 47.695555 & -122.791752 & 515626 & 5282484 \\
\hline 1167 & 66 & 47.704377 & -122.916251 & 506283 & 5283447 & 79 & 1261 & 6040 & 47.695472 & -122.790414 & 515726 & 5282475 \\
\hline 1168 & $* 534$ & 47.704403 & -122.914883 & 506386 & 5283450 & 71 & 1262 & 47 & 47.695446 & -122.789310 & 515809 & 5282473 \\
\hline 1169 & A032 & 47.704397 & -122.913529 & 506487 & 5283450 & 76 & 1263 & 6046 & 47.695462 & -122.787939 & 515912 & 5282475 \\
\hline 1170 & 65 & 47.704292 & -122.912337 & 506577 & 5283438 & 91 & 1264 & 42 & 47.695401 & -122.786556 & 516015 & 5282468 \\
\hline 1171 & A034 & 47.703627 & -122.910962 & 506680 & 5283364 & 82 & 1265 & 44 & 47.695249 & -122.785059 & 516128 & 5282452 \\
\hline 1172 & $* 535$ & 47.702583 & -122.909846 & 506764 & 5283248 & 64 & 1266 & $* 541$ & 47.695103 & -122.783713 & 516229 & 5282436 \\
\hline 1173 & $\bullet 7623$ & 47.701483 & -122.908979 & 506829 & 5283126 & 58 & 1268 & 49 & 47.694881 & -122.781550 & 516391 & 5282412 \\
\hline 1174 & A048 & 47.699906 & -122.907999 & 506903 & 5282951 & 54 & 1269 & $* 542$ & 47.694671 & -122.779971 & 516510 & 5282389 \\
\hline 1175 & 6043 & 47.698272 & -122.907183 & 506964 & 5282769 & 49 & 1270 & 39 & 47.693830 & -122.778505 & 516620 & 5282295 \\
\hline
\end{tabular}




\begin{tabular}{|c|c|c|c|c|c|}
\hline 1271 & 6042 & 47.693937 & -122.777308 & 516710 & 5282308 \\
\hline 1272 & A128 & 47.693681 & -122.775504 & 516845 & 5282279 \\
\hline 1273 & A129 & 47.693879 & -122.774456 & 516924 & 5282302 \\
\hline 1274 & A130 & 47.693457 & -122.773502 & 516996 & 5282255 \\
\hline 1275 & A131 & 47.693390 & -122.772608 & 517063 & 5282248 \\
\hline 1293 & $* 543$ & 47.689534 & -122.745028 & 519134 & 5281826 \\
\hline 1294 & $* 544$ & 47.689885 & -122.743775 & 519227 & 5281865 \\
\hline 1295 & $* 545$ & 47.690375 & -122.742806 & 519300 & 5281920 \\
\hline 1296 & $\bullet 7446$ & 47.690419 & -122.741529 & 519396 & 5281925 \\
\hline 1297 & A037 & 47.690324 & -122.740223 & 519494 & 5281915 \\
\hline 1298 & $* 546$ & 47.690322 & -122.738838 & 519598 & 5281915 \\
\hline 1299 & $* 651$ & 47.690331 & -122.737442 & 519703 & 5281916 \\
\hline 1300 & 7450 & 47.690281 & -122.736188 & 519797 & 5281911 \\
\hline 1300 & A036 & 47.690281 & -122.736188 & 519797 & 5281911 \\
\hline 1301 & $* 548$ & 47.690311 & -122.734996 & 519886 & 5281915 \\
\hline 1302 & 7594 & 47.689018 & -122.733900 & 519969 & 5281771 \\
\hline 1303 & 639 & 47.687985 & -122.732567 & 520069 & 5281657 \\
\hline 1304 & 7282 & 47.686759 & -122.731165 & 520175 & 5281521 \\
\hline 1305 & 638 & 47.684540 & -122.729626 & 520291 & 5281275 \\
\hline 1306 & 86 & 47.684609 & -122.728251 & 520394 & 5281283 \\
\hline 1307 & 7591 & 47.685177 & -122.727420 & 520457 & 5281346 \\
\hline 1308 & 7344 & 47.684391 & -122.726104 & 520556 & 5281259 \\
\hline 1309 & 85 & 47.683556 & -122.724700 & 520661 & 5281166 \\
\hline 1310 & 84 & 47.682632 & -122.723152 & 520778 & 5281064 \\
\hline 1311 & 83 & 47.682054 & -122.721719 & 520886 & 5281000 \\
\hline 1313 & 7358 & 47.681480 & -122.718981 & 521091 & 5280937 \\
\hline 1314 & 7328 & 47.680051 & -122.718363 & 521138 & 5280779 \\
\hline 1316 & 82 & 47.678869 & -122.715280 & 521370 & 5280648 \\
\hline 1317 & 81 & 47.678156 & -122.713612 & 521496 & 5280569 \\
\hline 1318 & 7343 & 47.678365 & -122.712447 & 521583 & 5280593 \\
\hline 1319 & 80 & 47.678747 & -122.711151 & 521680 & 5280636 \\
\hline 1320 & 7607 & 47.679026 & -122.709862 & 521777 & 5280667 \\
\hline 1321 & 79 & 47.679406 & -122.708506 & 521879 & 5280710 \\
\hline 1322 & 7322 & 47.679361 & -122.707245 & 521973 & 5280705 \\
\hline 1323 & 78 & 47.679314 & -122.705878 & 522076 & 5280700 \\
\hline 1324 & $* 552$ & 47.679209 & -122.704569 & 522174 & 5280689 \\
\hline 1325 & A053 & 47.678757 & -122.703391 & 522263 & 5280639 \\
\hline 1326 & 7286 & 47.678491 & -122.702047 & 522364 & 5280610 \\
\hline 1327 & 77 & 47.678323 & -122.700742 & 522462 & 5280592 \\
\hline 1328 & 76 & 47.678301 & -122.699309 & 522569 & 5280590 \\
\hline 1329 & 75 & 47.678331 & -122.698023 & 522666 & 5280593 \\
\hline 1330 & 7454 & 47.678338 & -122.696678 & 522767 & 5280594 \\
\hline 1331 & A054 & 47.678361 & -122.695292 & 522871 & 5280597 \\
\hline 1332 & 641 & 47.678385 & -122.693957 & 522971 & 5280600 \\
\hline 1333 & 640 & 47.678429 & -122.692968 & 523045 & 5280606 \\
\hline
\end{tabular}

\begin{tabular}{|c|c|c|c|c|c|}
\hline 1335 & 7075 & 47.678520 & -122.690236 & 523250 & 5280617 \\
\hline 1336 & 111 & 47.679385 & -122.687996 & 523418 & 5280713 \\
\hline 1337 & 110 & 47.679505 & -122.686660 & 523518 & 5280727 \\
\hline 1338 & 109 & 47.679404 & -122.685219 & 523626 & 5280716 \\
\hline 1343 & •7294 & 47.679133 & -122.678547 & 524127 & 5280688 \\
\hline 1344 & $* 553$ & 47.679055 & -122.676964 & 524246 & 5280680 \\
\hline 1345 & 108 & 47.679135 & -122.675530 & 524354 & 5280689 \\
\hline 1346 & A044 & 47.678038 & -122.675315 & 524370 & 5280568 \\
\hline 1347 & 7099 & 47.677242 & -122.674089 & 524463 & 5280479 \\
\hline 1348 & 107 & 47.677493 & -122.672975 & 524546 & 5280508 \\
\hline 1349 & $* 554$ & 47.677395 & -122.671458 & 524660 & 5280497 \\
\hline 1350 & 106 & 47.677394 & -122.670197 & 524755 & 5280498 \\
\hline 1351 & 105 & 47.677288 & -122.668541 & 524879 & 5280486 \\
\hline 1352 & 104 & 47.676463 & -122.666301 & 525048 & 5280395 \\
\hline 1353 & 103 & 47.677995 & -122.665454 & 525111 & 5280566 \\
\hline 1354 & 102 & 47.677686 & -122.664201 & 525205 & 5280532 \\
\hline 1355 & $* 555$ & 47.678154 & -122.663237 & 525277 & 5280584 \\
\hline 1356 & 6051 & 47.677995 & -122.661942 & 525374 & 5280567 \\
\hline 1357 & 101 & 47.678013 & -122.659926 & 525525 & 5280570 \\
\hline 1358 & A055 & 47.678308 & -122.658161 & 525658 & 5280603 \\
\hline 1359 & 100 & 47.680233 & -122.658034 & 525666 & 5280817 \\
\hline 1361 & 7360 & 47.679618 & -122.655312 & 525871 & 5280750 \\
\hline 1362 & 98 & 47.679466 & -122.653529 & 526005 & 5280733 \\
\hline 1363 & 99 & 47.679743 & -122.651834 & 526132 & 5280765 \\
\hline 1364 & A075 & 47.679906 & -122.650946 & 526199 & 5280783 \\
\hline 1365 & 7285 & 47.678488 & -122.649782 & 526287 & 5280626 \\
\hline 1366 & 97 & 47.677609 & -122.648229 & 526404 & 5280529 \\
\hline 1367 & $* 556$ & 47.677597 & -122.646949 & 526500 & 5280528 \\
\hline 1368 & 96 & 47.677637 & -122.645947 & 526575 & 5280533 \\
\hline 1370 & 7070 & 47.676864 & -122.643560 & 526754 & 5280448 \\
\hline 1371 & A074 & 47.676698 & -122.641827 & 526885 & 5280430 \\
\hline 1372 & 95 & 47.676716 & -122.640426 & 526990 & 5280432 \\
\hline 1373 & 94 & 47.676713 & -122.639099 & 527089 & 5280432 \\
\hline 1374 & $\bullet 7298$ & 47.676645 & -122.637708 & 527194 & 5280425 \\
\hline 1379 & 93 & 47.677399 & -122.631582 & 527653 & 5280511 \\
\hline 1380 & A076 & 47.676916 & -122.629869 & 527782 & 5280458 \\
\hline 1381 & 92 & 47.675662 & -122.629041 & 527845 & 5280319 \\
\hline 1383 & $* 557$ & 47.674016 & -122.626642 & 528026 & 5280137 \\
\hline 1384 & 91 & 47.673392 & -122.625299 & 528127 & 5280068 \\
\hline 1385 & A077 & 47.672759 & -122.624081 & 528219 & 5279998 \\
\hline 1386 & 7615 & 47.672079 & -122.622805 & 528315 & 5279923 \\
\hline 1387 & 90 & 47.670922 & -122.620981 & 528452 & 5279795 \\
\hline 1387 & 6032 & 47.670922 & -122.620981 & 5280452 & 5279795 \\
\hline 1388 & 87 & 47.668951 & -122.619730 & 528547 & 5279577 \\
\hline 1389 & 89 & 47.668436 & -122.618487 & 528641 & 5279520 \\
\hline
\end{tabular}




\begin{tabular}{|c|c|c|c|c|c|}
\hline 1390 & 88 & 47.666933 & -122.617090 & 528747 & 5279353 \\
\hline 2000 & A039 & 47.666250 & -122.750040 & 518766 & 5279237 \\
\hline 2002 & 112 & 47.666111 & -122.746938 & 518999 & 5279222 \\
\hline 2004 & 113 & 47.665376 & -122.743843 & 519231 & 5279141 \\
\hline 2006 & 114 & 47.665122 & -122.741302 & 519422 & 5279113 \\
\hline 2008 & A038 & 47.664817 & -122.738633 & 519623 & 5279080 \\
\hline 2010 & 7342 & 47.664895 & -122.735853 & 519831 & 5279090 \\
\hline 2012 & 115 & 47.664859 & -122.733519 & 520007 & 5279086 \\
\hline 2014 & $* 549$ & 47.663542 & -122.730849 & 520208 & 5278941 \\
\hline 2016 & 7629 & 47.662724 & -122.728201 & 520407 & 5278850 \\
\hline 2018 & 116 & 47.662284 & -122.725651 & 520598 & 5278802 \\
\hline 2020 & A040 & 47.661682 & -122.722659 & 520823 & 5278736 \\
\hline 2022 & 117 & 47.661266 & -122.720260 & 521004 & 5278690 \\
\hline 2024 & $* 638$ & 47.660431 & -122.718093 & 521167 & 5278598 \\
\hline 2027 & 118 & 47.661939 & -122.713182 & 521535 & 5278767 \\
\hline 2029 & 7083 & 47.662501 & -122.710964 & 521701 & 5278830 \\
\hline 2032 & A041 & 47.665682 & -122.706626 & 522025 & 5279185 \\
\hline 2034 & 119 & 47.666556 & -122.704361 & 522195 & 5279283 \\
\hline 2036 & 7452 & 47.666573 & -122.701000 & 522447 & 5279286 \\
\hline 2039 & $* 551$ & 47.665307 & -122.697669 & 522698 & 5279146 \\
\hline 2041 & 120 & 47.665502 & -122.695525 & 522859 & 5279168 \\
\hline 2043 & A052 & 47.665478 & -122.693173 & 523035 & 5279166 \\
\hline 2045 & 6122 & 47.665059 & -122.690114 & 523265 & 5279121 \\
\hline 2048 & $* 561$ & 47.665020 & -122.684871 & 523659 & 5279118 \\
\hline 2050 & 121 & 47.664708 & -122.683069 & 523794 & 5279084 \\
\hline 2052 & 122 & 47.667660 & -122.680951 & 523952 & 5279412 \\
\hline 2053 & 123 & 47.670362 & -122.678736 & 524117 & 5279713 \\
\hline 2055 & 124 & 47.669560 & -122.675483 & 524362 & 5279625 \\
\hline 2057 & 9900 & 47.633010 & -122.664560 & 525199 & 5275575 \\
\hline 2058 & $* 560$ & 47.667404 & -122.671440 & 524666 & 5279387 \\
\hline 2060 & A042 & 47.667526 & -122.668890 & 524858 & 5279401 \\
\hline 2062 & 634 & 47.668602 & -122.667220 & 524982 & 5279521 \\
\hline 2064 & 6028 & 47.669440 & -122.663180 & 525285 & 5279616 \\
\hline 2067 & A043 & 47.666869 & -122.660932 & 525455 & 5279331 \\
\hline 2069 & 635 & 47.666845 & -122.657631 & 525703 & 5279329 \\
\hline 2071 & 6048 & 47.666791 & -122.655036 & 525898 & 5279324 \\
\hline 2073 & $* 559$ & 47.666781 & -122.652497 & 526089 & 5279324 \\
\hline 2075 & 125 & 47.667708 & -122.650242 & 526257 & 5279428 \\
\hline 2077 & A073 & 47.668653 & -122.647400 & 526470 & 5279534 \\
\hline 2079 & 6100 & 47.668568 & -122.644528 & 526686 & 5279525 \\
\hline 2081 & 126 & 47.668547 & -122.641570 & 526908 & 5279524 \\
\hline 2083 & $* 558$ & 47.666806 & -122.639312 & 527078 & 5279331 \\
\hline 2085 & 127 & 47.664856 & -122.636515 & 527289 & 5279115 \\
\hline 2087 & 7076 & 47.664861 & -122.634075 & 527473 & 5279117 \\
\hline 2089 & 128 & 47.664841 & -122.631200 & 527689 & 5279116 \\
\hline
\end{tabular}

\begin{tabular}{|c|c|c|c|c|c|}
\hline 2091 & 129 & 47.664837 & -122.628678 & 527878 & 5279116 \\
\hline 2093 & 130 & 47.664769 & -122.625853 & 528090 & 5279110 \\
\hline 2095 & 131 & 47.664715 & -122.623206 & 528289 & 5279105 \\
\hline 2097 & 132 & 47.664678 & -122.620815 & 528468 & 5279101 \\
\hline 2100 & 133 & 47.664364 & -122.615558 & 528863 & 5279068 \\
\hline 2120 & 134 & 47.662990 & -122.590248 & 530764 & 5278925 \\
\hline 2121 & $\bullet 7269$ & 47.663331 & -122.589228 & 530841 & 5278964 \\
\hline 2122 & *639 & 47.663558 & -122.588002 & 530932 & 5278989 \\
\hline 2123 & 135 & 47.663765 & -122.586630 & 531035 & 5279013 \\
\hline 2124 & A151 & 47.663750 & -122.585273 & 531137 & 5279012 \\
\hline 2125 & 136 & 47.664839 & -122.583286 & 531286 & 5279134 \\
\hline 2126 & 7271 & 47.665216 & -122.582848 & 531318 & 5279176 \\
\hline 2127 & $* 640$ & 47.665775 & -122.581256 & 531438 & 5279239 \\
\hline 2128 & 636 & 47.665407 & -122.579609 & 531561 & 5279198 \\
\hline 2129 & 637 & 47.665661 & -122.578479 & 531646 & 5279227 \\
\hline 2130 & 137 & 47.665512 & -122.577065 & 531752 & 5279211 \\
\hline 2131 & 138 & 47.665092 & -122.575804 & 531847 & 5279165 \\
\hline 2132 & $* 562$ & 47.665803 & -122.574594 & 531938 & 5279244 \\
\hline 2133 & 139 & 47.665914 & -122.573135 & 532047 & 5279257 \\
\hline 2134 & 140 & 47.665840 & -122.571794 & 532148 & 5279250 \\
\hline 2138 & A152 & 47.664806 & -122.566915 & 532515 & 5279137 \\
\hline 2139 & 141 & 47.664782 & -122.565446 & 532625 & 5279135 \\
\hline 2140 & 7445 & 47.664731 & -122.563895 & 532742 & 5279130 \\
\hline 2141 & $* 649$ & 47.664855 & -122.562678 & 532833 & 5279144 \\
\hline 2142 & 142 & 47.664887 & -122.561387 & 532930 & 5279148 \\
\hline 2143 & A149 & 47.664853 & -122.559717 & 533055 & 5279145 \\
\hline 2144 & $\bullet 7268$ & 47.664950 & -122.558336 & 533159 & 5279156 \\
\hline 2145 & $* 564$ & 47.664832 & -122.557166 & 533247 & 5279144 \\
\hline 2146 & 143 & 47.664889 & -122.555713 & 533356 & 5279151 \\
\hline 2147 & 144 & 47.664870 & -122.554377 & 533456 & 5279149 \\
\hline 2148 & 145 & 47.664846 & -122.553020 & 533558 & 5279147 \\
\hline 2149 & 146 & 47.664847 & -122.551693 & 533658 & 5279148 \\
\hline 2150 & 147 & 47.664874 & -122.550346 & 533759 & 5279151 \\
\hline 2151 & 148 & 47.664873 & -122.548962 & 533863 & 5279152 \\
\hline 2152 & 149 & 47.664875 & -122.547653 & 533961 & 5279153 \\
\hline 2153 & 150 & 47.664899 & -122.546276 & 534064 & 5279156 \\
\hline 2154 & 151 & 47.664880 & -122.544890 & 534168 & 5279154 \\
\hline 2155 & 152 & 47.664893 & -122.543551 & 534269 & 5279156 \\
\hline 2156 & 153 & 47.664901 & -122.542206 & 534370 & 5279158 \\
\hline 2157 & 154 & 47.664918 & -122.540862 & 534471 & 5279160 \\
\hline 2158 & 155 & 47.664900 & -122.539502 & 534573 & 5279159 \\
\hline 2159 & 156 & 47.664866 & -122.538192 & 534671 & 5279156 \\
\hline 2160 & 157 & 47.664853 & -122.536870 & 534771 & 5279155 \\
\hline 2161 & 158 & 47.664849 & -122.535503 & 534873 & 5279155 \\
\hline 2162 & 159 & 47.664885 & -122.534213 & 534970 & 5279160 \\
\hline
\end{tabular}




\begin{tabular}{|c|c|c|c|c|c|c|c|c|c|c|c|c|}
\hline 2163 & 160 & 47.664841 & -122.532886 & 535070 & 5279155 & 43 & 2250 & 199 & 47.666498 & -122.417672 & 543718 & 5279398 \\
\hline 2164 & 161 & 47.664812 & -122.531556 & 535170 & 5279153 & 26 & 2251 & 389 & 47.666102 & -122.416194 & 543829 & 5279355 \\
\hline 2165 & 162 & 47.664802 & -122.530238 & 535268 & 5279152 & 16 & 2252 & 390 & 47.665755 & -122.414611 & 543949 & 5279317 \\
\hline 2166 & 163 & 47.664856 & -122.528757 & 535380 & 5279159 & 17 & 2253 & 391 & 47.665357 & -122.413117 & 544061 & 5279274 \\
\hline 2167 & 164 & 47.664721 & -122.527595 & 535467 & 5279145 & 33 & 2254 & 392 & 47.665074 & -122.411718 & 544166 & 5279243 \\
\hline 2168 & 165 & 47.664795 & -122.526162 & 535575 & 5279153 & 47 & 2255 & 393 & 47.664804 & -122.410142 & 544285 & 5279214 \\
\hline 2169 & $* 566$ & 47.664794 & -122.524960 & 535665 & 5279154 & 54 & 2256 & 394 & 47.664432 & -122.408669 & 544396 & 5279174 \\
\hline 2170 & A150 & 47.664743 & -122.523726 & 535757 & 5279149 & 58 & 2257 & 395 & 47.664150 & -122.407260 & 544502 & 5279143 \\
\hline 2171 & A155 & 47.664768 & -122.522385 & 535858 & 5279152 & 68 & 2258 & 396 & 47.663957 & -122.406176 & 544583 & 5279122 \\
\hline 2172 & A153 & 47.664658 & -122.520841 & 535974 & 5279141 & 79 & 2259 & 397 & 47.663769 & -122.404900 & 544679 & 5279102 \\
\hline 2173 & 166 & 47.664692 & -122.519668 & 536062 & 5279145 & 78 & 2260 & 398 & 47.670031 & -122.403968 & 544744 & 5279799 \\
\hline 2174 & 167 & 47.664733 & -122.518217 & 536171 & 5279150 & 58 & 2261 & $\mathrm{O} 312$ & 47.670146 & -122.402791 & 544832 & 5279812 \\
\hline 2175 & 168 & 47.664725 & -122.516972 & 536265 & 5279150 & 47 & 2262 & $\bullet 7595$ & 47.670294 & -122.401406 & 544936 & 5279829 \\
\hline 2176 & 169 & 47.664671 & -122.515771 & 536355 & 5279144 & 52 & 2263 & 212 & 47.670249 & -122.399812 & 545056 & 5279825 \\
\hline 2177 & 170 & 47.664725 & -122.514345 & 536462 & 5279151 & 49 & 2264 & $* 573$ & 47.670499 & -122.398666 & 545142 & 5279854 \\
\hline 2178 & 171 & 47.664692 & -122.512899 & 536570 & 5279148 & 42 & 2265 & 7619 & 47.670661 & -122.397478 & 545231 & 5279872 \\
\hline 2179 & 172 & 47.664690 & -122.511692 & 536661 & 5279148 & 49 & 2266 & 7316 & 47.670967 & -122.396260 & 545322 & 5279907 \\
\hline 2180 & 173 & 47.664690 & -122.510191 & 536774 & 5279149 & 52 & 2267 & $\mathrm{O} 271$ & 47.671102 & -122.394909 & 545423 & 5279923 \\
\hline 2181 & 174 & 47.664697 & -122.508906 & 536870 & 5279151 & 54 & 2268 & 7355 & 47.671029 & -122.393237 & 545549 & 5279916 \\
\hline 2182 & 175 & 47.664697 & -122.507599 & 536968 & 5279151 & 53 & 2269 & $* 642$ & 47.670808 & -122.392004 & 545641 & 5279892 \\
\hline 2183 & 176 & 47.664717 & -122.506398 & 537058 & 5279154 & 49 & 2270 & 7292 & 47.670808 & -122.390831 & 545730 & 5279893 \\
\hline 2184 & 177 & 47.663493 & -122.504801 & 537179 & 5279019 & 35 & 2271 & 213 & 47.671154 & -122.389451 & 545833 & 5279932 \\
\hline 2185 & 178 & 47.663201 & -122.503579 & 537271 & 5278987 & 39 & 2272 & 7303 & 47.670511 & -122.388182 & 545929 & 5279861 \\
\hline 2186 & 179 & 47.662621 & -122.501926 & 537396 & 5278923 & 38 & 2273 & 214 & 47.670790 & -122.388197 & 545927 & 5279892 \\
\hline 2187 & 180 & 47.662742 & -122.500548 & 537499 & 5278937 & 15 & 2274 & $\mathrm{O} 275$ & 47.671284 & -122.386340 & 546066 & 5279948 \\
\hline 2190 & D9 & 47.665783 & -122.493217 & 538047 & 5279279 & -56 & 2275 & $* 654$ & 47.670567 & -122.384207 & 546227 & 5279870 \\
\hline 2196 & $\mathrm{C} 1$ & 47.666000 & -122.485667 & 538614 & 5279307 & -232 & 2276 & 7351 & 47.670762 & -122.382568 & 546350 & 5279892 \\
\hline 2202 & A4 & 47.666333 & -122.477667 & 539214 & 5279348 & -250 & 2277 & 7352 & 47.671287 & -122.380764 & 546485 & 5279952 \\
\hline 2208 & $\mathrm{C} 3$ & 47.666500 & -122.470667 & 539740 & 5279370 & -225 & 2278 & $\mathrm{O} 281$ & 47.671573 & -122.379765 & 546560 & 5279984 \\
\hline 2214 & $\mathrm{~B} 2$ & 47.666167 & -122.463833 & 540253 & 5279336 & -228 & 2279 & $* 646$ & 47.670650 & -122.378245 & 546674 & 5279883 \\
\hline 2220 & D4 & 47.665833 & -122.455833 & 540854 & 5279303 & -247 & 2280 & 7598 & 47.670820 & -122.377386 & 546739 & 5279902 \\
\hline 2226 & D1 & 47.666500 & -122.440833 & 541979 & 5279386 & -70 & 2281 & $\mathrm{O} 262$ & 47.670975 & -122.375812 & 546857 & 5279920 \\
\hline 2232 & A8 & 47.666333 & -122.436667 & 542292 & 5279369 & -54 & 2283 & 7332 & 47.670845 & -122.373118 & 547059 & 5279907 \\
\hline 2238 & 187 & 47.661433 & -122.433998 & 542497 & 5278826 & 1 & 2283 & 215 & 47.670845 & -122.373118 & 547059 & 5279907 \\
\hline 2239 & 188 & 47.661142 & -122.433232 & 542554 & 5278794 & 3 & 2284 & $* 661$ & 47.670787 & -122.371884 & 547152 & 5279902 \\
\hline 2240 & 189 & 47.660821 & -122.431891 & 542655 & 5278759 & 4 & 2285 & 216 & 47.670843 & -122.370493 & 547256 & 5279909 \\
\hline 2241 & 190 & 47.660745 & -122.429326 & 542848 & 5278752 & 27 & 2286 & 7430 & 47.670584 & -122.369432 & 547336 & 5279881 \\
\hline 2242 & 191 & 47.660040 & -122.427862 & 542958 & 5278675 & 42 & 2287 & $\mathrm{O} 311$ & 47.670792 & -122.367961 & 547446 & 5279905 \\
\hline 2243 & 192 & 47.660154 & -122.426521 & 543059 & 5278688 & 48 & 2288 & 7434 & 47.670703 & -122.366609 & 547548 & 5279896 \\
\hline 2244 & 193 & 47.661002 & -122.425378 & 543144 & 5278783 & 53 & 2289 & 217 & 47.670601 & -122.365336 & 547644 & 5279885 \\
\hline 2245 & 194 & 47.661594 & -122.424012 & 543246 & 5278850 & 78 & 2290 & 7330 & 47.670799 & -122.363628 & 547772 & 5279908 \\
\hline 2246 & 195 & 47.662134 & -122.422356 & 543370 & 5278911 & 78 & 2291 & $* 660$ & 47.671151 & -122.362382 & 547865 & 5279948 \\
\hline 2247 & 196 & 47.663190 & -122.421253 & 543452 & 5279029 & 67 & 2292 & $\mathrm{O} 288$ & 47.670758 & -122.360993 & 547969 & 5279905 \\
\hline 2248 & 197 & 47.663784 & -122.420007 & 543545 & 5279095 & 83 & 2293 & $\mathrm{O} 317$ & 47.671301 & -122.360115 & 548035 & 5279966 \\
\hline 2249 & 198 & 47.666042 & -122.419325 & 543594 & 5279347 & 68 & 2294 & 7347 & 47.671330 & -122.358488 & 548157 & 5279970 \\
\hline
\end{tabular}




\begin{tabular}{|c|c|c|c|c|c|c|}
\hline 2295 & $* 572$ & 47.671281 & -122.357425 & 548237 & 5279965 & 91 \\
\hline 2296 & $\bullet 7618$ & 47.671045 & -122.355792 & 548360 & 5279940 & 104 \\
\hline 2297 & 218 & 47.670273 & -122.354099 & 548487 & 5279856 & 105 \\
\hline 2298 & 219 & 47.670341 & -122.353079 & 548564 & 5279864 & 119 \\
\hline 2299 & 220 & 47.670654 & -122.351442 & 548687 & 5279900 & 118 \\
\hline 2300 & 223 & 47.670955 & -122.350338 & 548769 & 5279934 & 98 \\
\hline 2301 & 222 & 47.671218 & -122.349491 & 548832 & 5279963 & 104 \\
\hline 2302 & 224 & 47.670803 & -122.348017 & 548944 & 5279918 & 85 \\
\hline 2303 & $\mathrm{O} 258$ & 47.670587 & -122.347127 & 549011 & 5279895 & 87 \\
\hline 2304 & 201 & 47.670587 & -122.346034 & 549093 & 5279896 & 79 \\
\hline 2305 & 200 & 47.670636 & -122.345093 & 549163 & 5279902 & 73 \\
\hline 2306 & O305 & 47.671562 & -122.342893 & 549327 & 5280006 & 55 \\
\hline 2307 & 202 & 47.670148 & -122.342097 & 549389 & 5279849 & 63 \\
\hline 2308 & $\mathrm{O} 257$ & 47.670090 & -122.340082 & 549540 & 5279844 & 57 \\
\hline 2309 & 203 & 47.668732 & -122.338660 & 549648 & 5279694 & 72 \\
\hline 2310 & 7087 & 47.668525 & -122.337407 & 549742 & 5279672 & 68 \\
\hline 2311 & $* 575$ & 47.668973 & -122.336217 & 549831 & 5279722 & 71 \\
\hline 2312 & 7043 & 47.668595 & -122.334842 & 549935 & 5279681 & 75 \\
\hline 2313 & $\mathrm{O} 274$ & 47.669014 & -122.333798 & 550013 & 5279729 & 87 \\
\hline 2314 & O067 & 47.668431 & -122.331974 & 550150 & 5279665 & 88 \\
\hline 2315 & 7048 & 47.669415 & -122.330721 & 550243 & 5279775 & 82 \\
\hline 2316 & $* 578$ & 47.669280 & -122.329152 & 550361 & 5279761 & 88 \\
\hline 2317 & 204 & 47.669224 & -122.327879 & 550457 & 5279756 & 92 \\
\hline 2318 & 7101 & 47.669650 & -122.326693 & 550545 & 5279804 & 91 \\
\hline 2319 & O304 & 47.669633 & -122.325401 & 550642 & 5279803 & 96 \\
\hline 2320 & 7060 & 47.669127 & -122.324313 & 550725 & 5279747 & 83 \\
\hline 2321 & O306 & 47.669121 & -122.323020 & 550822 & 5279747 & 70 \\
\hline 2322 & & 47.669215 & -122.321182 & 550960 & 5279759 & 65 \\
\hline 2323 & $* 579$ & 47.669439 & -122.319747 & 551067 & 5279785 & 58 \\
\hline 2324 & $\mathrm{O} 277$ & 47.669145 & -122.318568 & 551156 & 5279753 & 63 \\
\hline 2325 & $\mathrm{O} 278$ & 47.669352 & -122.317073 & 551268 & 5279777 & 60 \\
\hline 2326 & 7104 & 47.669168 & -122.315911 & 551355 & 5279757 & 60 \\
\hline 2327 & 205 & 47.669609 & -122.314763 & 551441 & 5279807 & 71 \\
\hline 2328 & 7444 & 47.669573 & -122.313652 & 551524 & 5279804 & 65 \\
\hline 2329 & $\mathrm{O} 280$ & 47.669835 & -122.312372 & 551620 & 5279834 & 76 \\
\hline 2330 & $* 580$ & 47.669288 & -122.310928 & 551729 & 5279774 & 84 \\
\hline 2331 & 206 & 47.669336 & -122.309725 & 551819 & 5279780 & 79 \\
\hline 2332 & 7044 & 47.669536 & -122.307943 & 551953 & 5279804 & 70 \\
\hline 2333 & $\mathrm{O} 273$ & 47.669448 & -122.306654 & 552050 & 5279795 & 58 \\
\hline 2334 & 7107 & 47.670195 & -122.305666 & 552123 & 5279878 & 48 \\
\hline 2335 & 207 & 47.669979 & -122.304164 & 552236 & 5279855 & 45 \\
\hline 2336 & 208 & 47.670631 & -122.302829 & 552336 & 5279929 & 53 \\
\hline 2337 & $\mathrm{O} 272$ & 47.672017 & -122.301748 & 552416 & 5280083 & 53 \\
\hline 2338 & $\bullet 7610$ & 47.672049 & -122.300207 & 552531 & 5280088 & 49 \\
\hline 2339 & $* 581$ & 47.672045 & -122.298882 & 552631 & 5280089 & 53 \\
\hline
\end{tabular}

\begin{tabular}{|c|c|c|c|c|c|}
\hline 2340 & $\mathrm{O} 263$ & 47.672202 & -122.297637 & 552724 & 5280107 \\
\hline 2341 & $* 582$ & 47.671953 & -122.296071 & 552842 & 5280080 \\
\hline 2342 & 7077 & 47.672292 & -122.294847 & 552933 & 5280119 \\
\hline 2343 & $* 583$ & 47.672127 & -122.293566 & 553030 & 5280101 \\
\hline 2344 & 7058 & 47.672212 & -122.292537 & 553107 & 5280111 \\
\hline 2345 & O318 & 47.671834 & -122.290934 & 553228 & 5280070 \\
\hline 2346 & 7045 & 47.672247 & -122.289497 & 553335 & 5280117 \\
\hline 2347 & 209 & 47.672257 & -122.288531 & 553408 & 5280119 \\
\hline 2348 & $* 584$ & 47.672222 & -122.287306 & 553500 & 5280116 \\
\hline 2349 & 210 & 47.672209 & -122.285638 & 553625 & 5280116 \\
\hline 2350 & 7100 & 47.672030 & -122.284203 & 553733 & 5280097 \\
\hline 2351 & O308 & 47.672347 & -122.282914 & 553829 & 5280133 \\
\hline 2352 & 7068 & 47.672013 & -122.281295 & 553951 & 5280097 \\
\hline 2353 & $* 586$ & 47.671963 & -122.280250 & 554029 & 5280092 \\
\hline 2354 & 7085 & 47.671969 & -122.278931 & 554128 & 5280094 \\
\hline 2355 & $* 587$ & 47.672001 & -122.277699 & 554221 & 5280098 \\
\hline 2356 & O286 & 47.672244 & -122.276164 & 554336 & 5280126 \\
\hline 2357 & 211 & 47.671663 & -122.274716 & 554445 & 5280063 \\
\hline 2358 & 7112 & 47.672043 & -122.273702 & 554521 & 5280106 \\
\hline 2359 & $* 588$ & 47.671958 & -122.272162 & 554637 & 5280097 \\
\hline 2360 & $\bullet 7626$ & 47.671987 & -122.270937 & 554729 & 5280101 \\
\hline 2361 & 407 & 47.672196 & -122.269970 & 554801 & 5280125 \\
\hline 2362 & 408 & 47.671942 & -122.268751 & 554893 & 5280098 \\
\hline 2363 & 7109 & 47.672702 & -122.266721 & 555044 & 5280184 \\
\hline 2364 & $* 590$ & 47.673117 & -122.265778 & 555115 & 5280231 \\
\hline 2365 & $\mathrm{O} 285$ & 47.672951 & -122.264601 & 555203 & 5280213 \\
\hline 2366 & 7064 & 47.672612 & -122.263393 & 555294 & 5280176 \\
\hline 2367 & O310 & 47.672744 & -122.262067 & 555394 & 5280192 \\
\hline 2368 & 7061 & 47.672801 & -122.260491 & 555512 & 5280199 \\
\hline 2369 & $* 591$ & 47.672717 & -122.259389 & 555595 & 5280191 \\
\hline 2370 & 7059 & 47.672479 & -122.257953 & 555703 & 5280165 \\
\hline 2371 & $* 592$ & 47.672973 & -122.256860 & 555784 & 5280221 \\
\hline 2372 & 7074 & 47.672797 & -122.255241 & 555906 & 5280203 \\
\hline 2373 & O307 & 47.673944 & -122.253273 & 556052 & 5280332 \\
\hline 2374 & 402 & 47.674428 & -122.251425 & 556191 & 5280387 \\
\hline 2375 & 401 & 47.675357 & -122.250599 & 556252 & 5280491 \\
\hline 2376 & 406 & 47.677825 & -122.249057 & 556365 & 5280766 \\
\hline 2377 & 405 & 47.678862 & -122.248080 & 556437 & 5280882 \\
\hline 2378 & 404 & 47.680428 & -122.247081 & 556510 & 5281057 \\
\hline 2409 & $* 644$ & 47.661072 & -122.206006 & 559615 & 5278936 \\
\hline 2410 & •7601 & 47.661199 & -122.204631 & 559718 & 5278951 \\
\hline 2411 & A132 & 47.660904 & -122.203322 & 559817 & 5278920 \\
\hline 2412 & 7336 & 47.660968 & -122.201947 & 559920 & 5278928 \\
\hline 2413 & 420 & 47.661171 & -122.200359 & 560039 & 5278952 \\
\hline 2414 & $* 604$ & 47.660898 & -122.199466 & 560106 & 5278922 \\
\hline
\end{tabular}




\begin{tabular}{|c|c|c|c|c|c|}
\hline 2415 & 421 & 47.661206 & -122.198068 & 560211 & 5278957 \\
\hline 2416 & 7287 & 47.661423 & -122.196727 & 560311 & 5278982 \\
\hline 2417 & A134 & 47.660932 & -122.195546 & 560401 & 5278929 \\
\hline 2418 & 7306 & 47.660907 & -122.193955 & 560520 & 5278927 \\
\hline 2419 & $* 602$ & 47.661041 & -122.192379 & 560638 & 5278943 \\
\hline 2420 & 7296 & 47.661292 & -122.191358 & 560715 & 5278972 \\
\hline 2421 & A135 & 47.661130 & -122.190124 & 560807 & 5278955 \\
\hline 2422 & 422 & 47.661158 & -122.188751 & 560911 & 5278959 \\
\hline 2423 & 417 & 47.661041 & -122.187654 & 560993 & 5278947 \\
\hline 2424 & 418 & 47.661114 & -122.186185 & 561103 & 5278956 \\
\hline 2425 & 419 & 47.660994 & -122.184961 & 561195 & 5278944 \\
\hline 2426 & 416 & 47.660962 & -122.183590 & 561298 & 5278941 \\
\hline 2427 & 415 & 47.660917 & -122.182338 & 561392 & 5278937 \\
\hline 2428 & 414 & 47.660884 & -122.181033 & 561490 & 5278935 \\
\hline 2429 & 444 & 47.660120 & -122.179312 & & 5278851 \\
\hline 2430 & 443 & 47.660151 & -122.178014 & 561718 & 5278856 \\
\hline 2431 & 441 & 47.660724 & -122.176313 & 561845 & 5278921 \\
\hline 2432 & 440 & 47.660725 & -122.175019 & 5619 & 5278922 \\
\hline 2433 & 439 & 47.660670 & -122.173254 & 075 & 5278917 \\
\hline 2434 & 445 & 47.660668 & -122.172179 & 562155 & 5278918 \\
\hline 2435 & 442 & 47.659862 & -122.171153 & 562233 & 5278829 \\
\hline 2436 & 438 & 47.65 & -122.16 & 562 & 5278822 \\
\hline 2437 & 434 & 47.660706 & -122.168410 & 562438 & 5278925 \\
\hline 2438 & 437 & 47.660771 & -122.167133 & 562534 & 5278933 \\
\hline 2439 & 428 & 47.660772 & -122.165783 & 562635 & 5278935 \\
\hline 2440 & 431 & 47.6 & -122. & 735 & 5278929 \\
\hline 2441 & A136 & 47.661654 & -122.163515 & 562805 & 5279034 \\
\hline 2442 & 436 & 47.663055 & -122.161354 & 562965 & 5279192 \\
\hline 2443 & 7604 & 47.663906 & -122.160202 & 563051 & 5279287 \\
\hline 2444 & $* 603$ & 47.665269 & -122.158743 & 563159 & 5279440 \\
\hline 2445 & 427 & 47.667272 & -122.157827 & 563225 & 5279663 \\
\hline 2446 & A 140 & 47.667358 & -122.156610 & 563316 & 5279674 \\
\hline 2447 & 7357 & 47.667278 & -122.155403 & 563407 & 5279666 \\
\hline 2448 & 432 & 47.666636 & -122.153872 & 563523 & 5279596 \\
\hline 2449 & $* 600$ & 47.665551 & -122.152273 & 563644 & 5279477 \\
\hline 2450 & 433 & 47.665727 & -122.150621 & 563768 & 5279498 \\
\hline 2451 & $\bullet 7599$ & 47.666493 & -122.149567 & 563846 & 5279584 \\
\hline 2452 & A139 & 47.666109 & -122.148306 & 563941 & 5279542 \\
\hline 2453 & 430 & 47.666253 & -122.147132 & 564029 & 5279559 \\
\hline 2454 & $* 599$ & 47.666506 & -122.145909 & 564121 & 5279588 \\
\hline 2455 & 7317 & 47.666835 & -122.144210 & 564248 & 5279626 \\
\hline 2456 & 429 & 47.667362 & -122.142480 & 564377 & 5279686 \\
\hline 2457 & A 138 & 47.667925 & -122.141136 & 564477 & 5279750 \\
\hline 2458 & 426 & 47.667917 & -122.139976 & 564564 & 5279750 \\
\hline 2459 & 7320 & 47.668319 & -122.138940 & 564642 & 5279795 \\
\hline
\end{tabular}

\begin{tabular}{|c|c|c|c|c|c|}
\hline 2460 & $* 601$ & 47.668163 & -122.137604 & 564742 & 5279779 \\
\hline 2461 & 7447 & 47.668118 & -122.135802 & 564877 & 5279776 \\
\hline 2462 & A137 & 47.666038 & -122.134970 & 564942 & 5279545 \\
\hline 2463 & 435 & 47.665867 & -122.133460 & 565056 & 5279527 \\
\hline 2464 & 493 & 47.663863 & -122.132714 & 565115 & 5279305 \\
\hline 2465 & $* 657$ & 47.664036 & -122.131329 & 565218 & 5279326 \\
\hline 2466 & 498 & 47.663942 & -122.129776 & 565335 & 5279317 \\
\hline 2467 & 7338 & 47.663118 & -122.127877 & 565479 & 5279227 \\
\hline 2468 & A102 & 47.661607 & -122.126252 & 565603 & 5279060 \\
\hline 2469 & 492 & 47.661900 & -122.124999 & 565602 & 5279093 \\
\hline 2470 & 491 & 47.662372 & -122.123893 & 565779 & 5279147 \\
\hline 2471 & 490 & 47.662747 & -122.122670 & 565870 & 5279190 \\
\hline 2472 & 489 & 47.663158 & -122.121397 & 565965 & 5279237 \\
\hline 2473 & 488 & 47.663501 & -122.120588 & 566025 & 5279275 \\
\hline 2474 & 487 & 47.663632 & -122.119210 & 566129 & 5279291 \\
\hline 2475 & 486 & 47.663955 & -122.117805 & 566234 & 5279328 \\
\hline 2476 & 485 & 47.663960 & -122.116461 & 566335 & 5279330 \\
\hline 2477 & 484 & 47.664014 & -122.115360 & 566417 & 5279337 \\
\hline 2478 & 483 & 47.663922 & -122.113933 & 566525 & 5279328 \\
\hline 2479 & 482 & 47.663916 & -122.112388 & 566641 & 5279329 \\
\hline 2480 & 481 & 47.664099 & -122.111001 & 566744 & 5279350 \\
\hline 2481 & 480 & 47.664013 & -122.109566 & 566852 & 5279342 \\
\hline 2482 & 479 & 47.664010 & -122.108328 & 566945 & 5279343 \\
\hline 2483 & 478 & 47.664005 & -122.107032 & 567043 & 5279343 \\
\hline 2484 & 477 & 47.663986 & -122.105670 & 567145 & 5279342 \\
\hline 2485 & 476 & 47.663709 & -122.104323 & 567246 & 5279313 \\
\hline 2486 & 452 & 47.663919 & -122.103027 & 567343 & 5279337 \\
\hline 2487 & 453 & 47.663884 & -122.101737 & 567440 & 5279334 \\
\hline 2488 & 454 & 47.663923 & -122.100470 & 567535 & 5279340 \\
\hline 2489 & 455 & 47.664433 & -122.098784 & 567661 & 5279398 \\
\hline 2490 & 451 & 47.663704 & -122.097513 & 567758 & 5279318 \\
\hline 2491 & $\bullet 7359$ & 47.662819 & -122.096633 & 567825 & 5279220 \\
\hline 2492 & A101 & 47.662032 & -122.095773 & 567890 & 5279134 \\
\hline 2493 & 463 & 47.660536 & -122.094525 & 567986 & 5278968 \\
\hline 2494 & 7108 & 47.659494 & -122.093438 & 568069 & 5278854 \\
\hline 2495 & 450 & 47.658792 & -122.092350 & 568152 & 5278777 \\
\hline 2496 & 462 & 47.658055 & -122.090755 & 568272 & 5278696 \\
\hline 2497 & A100 & 47.657601 & -122.089321 & 568381 & 5278647 \\
\hline 2498 & 7333 & 47.657286 & -122.087536 & 568515 & 5278613 \\
\hline 2499 & 459 & 47.656963 & -122.086168 & 568618 & 5278579 \\
\hline 2500 & 448 & 47.656584 & -122.084762 & 568724 & 5278538 \\
\hline 2501 & 461 & 47.656428 & -122.083582 & 568813 & 5278522 \\
\hline 2502 & 7067 & 47.656283 & -122.082349 & 568906 & 5278507 \\
\hline 2503 & A106 & 47.656055 & -122.081140 & 568997 & 5278482 \\
\hline 2504 & 460 & 47.655915 & -122.079932 & 569088 & 5278468 \\
\hline
\end{tabular}




\begin{tabular}{|c|c|c|c|c|c|}
\hline 2505 & 549 & 47.655641 & -122.078792 & 569174 & 5278438 \\
\hline 2506 & 7273 & 47.655609 & -122.077436 & 569276 & 5278436 \\
\hline 2507 & 458 & 47.655302 & -122.076160 & 569372 & 5278403 \\
\hline 2508 & A 105 & 47.655158 & -122.074752 & 569478 & 5278388 \\
\hline 2509 & 457 & 47.655072 & -122.073296 & 569587 & 5278380 \\
\hline 2510 & 7277 & 47.655643 & -122.072147 & 569673 & 5278445 \\
\hline 2511 & 447 & 47.656197 & -122.070972 & 569760 & 5278507 \\
\hline 2512 & 456 & 47.660258 & -122.069901 & 569835 & 5278959 \\
\hline 2513 & A103 & 47.660367 & -122.068665 & 569928 & 5278973 \\
\hline 2514 & 7321 & 47.660264 & -122.067149 & 570042 & 5278963 \\
\hline 2515 & A104 & 47.660268 & -122.065748 & 570147 & 5278964 \\
\hline 2516 & 548 & 47.660704 & -122.064311 & 570254 & 5279014 \\
\hline 2517 & 7348 & 47.671214 & -122.062889 & 570347 & 5280183 \\
\hline 2518 & 494 & 47.671306 & -122.061466 & 570454 & 5280195 \\
\hline 2519 & 496 & 1372 & -122.060139 & & 5280203 \\
\hline 2520 & A116 & 47.671333 & -122.058828 & 570652 & 5280200 \\
\hline 2521 & 449 & 47.671238 & -122.057426 & 570757 & 5280191 \\
\hline 2522 & 7462 & 47.671109 & -122.056029 & 570862 & 5280178 \\
\hline 2523 & 495 & 47.670828 & -122.05 & 0963 & 5280148 \\
\hline 2524 & A117 & 47.670522 & -122.053435 & 571058 & 5280115 \\
\hline 2525 & $\bullet 7280$ & 47.669515 & -122.051992 & 571167 & 5280005 \\
\hline 2526 & 497 & 47.669218 & -122.0 & 571262 & 5279973 \\
\hline 2527 & 464 & 47.668936 & -122.049426 & 571361 & 5279943 \\
\hline 2528 & 465 & 47.668690 & -122.048101 & 571461 & 5279916 \\
\hline 2529 & 466 & 47.668416 & -122.046623 & 571572 & 5279887 \\
\hline 2530 & 510 & 8137 & -122.0 & 571672 & 9858 \\
\hline 2531 & 513 & 47.667940 & -122.043939 & 571774 & 5279837 \\
\hline 2532 & 516 & 47.667704 & -122.042608 & 571874 & 5279812 \\
\hline 2533 & 515 & 47.667434 & -122.041250 & 571977 & 5279783 \\
\hline 2534 & 446 & 47.667183 & -122.039964 & 572073 & 5279756 \\
\hline 2535 & A 120 & 47.666968 & -122.038444 & 572188 & 5279734 \\
\hline 2536 & 7302 & 47.666679 & -122.036683 & 572320 & 5279704 \\
\hline 2537 & 514 & 47.666414 & -122.035321 & 572423 & 5279675 \\
\hline 2538 & 550 & 47.666058 & -122.034007 & 572522 & 5279637 \\
\hline 2539 & 519 & 47.665705 & -122.032593 & 572629 & 5279599 \\
\hline 2540 & 7453 & 47.665474 & -122.031218 & 572732 & 5279575 \\
\hline 2541 & A121 & 47.665263 & -122.030083 & 572818 & 5279552 \\
\hline 2542 & 518 & 47.665063 & -122.028911 & 572906 & 5279531 \\
\hline 2543 & 500 & 47.664809 & -122.027628 & 573003 & 5279504 \\
\hline 2544 & 7362 & 47.664637 & -122.026321 & 573101 & 5279486 \\
\hline 2545 & 522 & 47.665034 & -122.024893 & 573208 & 5279532 \\
\hline 2546 & A113 & 47.665980 & -122.023698 & 573296 & 5279638 \\
\hline 2547 & 399 & 47.666728 & -122.022283 & 573401 & 5279722 \\
\hline 2548 & 7040 & 47.667966 & -122.021380 & 573468 & 5279861 \\
\hline 2549 & A114 & 47.671358 & -122.019231 & 573624 & 5280240 \\
\hline
\end{tabular}

\begin{tabular}{|c|c|c|c|c|c|}
\hline 2550 & 517 & 47.674974 & -122.018073 & 573706 & 5280643 \\
\hline 2551 & A115 & 47.674957 & -122.016758 & 573805 & 5280642 \\
\hline 2552 & 7276 & 47.675014 & -122.015458 & 573902 & 5280650 \\
\hline 2553 & 629 & 47.674997 & -122.014113 & 574003 & 5280649 \\
\hline 2554 & 520 & 47.674874 & -122.012693 & 574110 & 5280637 \\
\hline 2555 & A112 & 47.674794 & -122.011371 & 574209 & 5280629 \\
\hline 2556 & 7069 & 47.674748 & -122.010038 & 574309 & 5280625 \\
\hline 2557 & 521 & 47.674688 & -122.008758 & 574406 & 5280620 \\
\hline 2558 & 630 & 47.674635 & -122.007464 & 574503 & 5280615 \\
\hline 2559 & A098 & 47.674538 & -122.006108 & 574605 & 5280606 \\
\hline 2560 & 7046 & 47.674508 & -122.004668 & 574713 & 5280604 \\
\hline 2561 & A099 & 47.674374 & -122.003442 & 574805 & 5280590 \\
\hline 2562 & 523 & 47.674253 & -122.002126 & 574904 & 5280578 \\
\hline 2563 & 626 & 47.674193 & -122.000813 & 575003 & 5280573 \\
\hline 2564 & 7080 & 47.674048 & -121.999494 & 575102 & 5280558 \\
\hline 2565 & 524 & 47.674001 & -121.998152 & 575203 & 5280554 \\
\hline 2566 & A097 & 47.673856 & -121.996816 & 575303 & 5280539 \\
\hline 2567 & 525 & 47.673827 & -121.995502 & 575402 & 5280537 \\
\hline 2568 & 7049 & 47.673693 & -121.994217 & 575498 & 5280524 \\
\hline 2569 & 625 & 47.673595 & -121.992979 & 575591 & 5280514 \\
\hline 2570 & $\bullet 7289$ & 47.673382 & -121.991437 & 575708 & 5280492 \\
\hline 2580 & A107 & 47.673555 & -121.978923 & 576647 & 5280523 \\
\hline 2581 & 7466 & 47.673455 & -121.977323 & 576767 & 5280514 \\
\hline 2582 & 511 & 47.673467 & -121.976017 & 576865 & 5280516 \\
\hline 2583 & 611 & 47.673464 & -121.974782 & 576958 & 5280517 \\
\hline 2584 & 512 & 47.673428 & -121.973462 & 577057 & 5280515 \\
\hline 2585 & 7451 & 47.673417 & -121.972115 & 577158 & 5280515 \\
\hline 2586 & A108 & 47.673419 & -121.970801 & 577257 & 5280516 \\
\hline 2587 & 507 & 47.673404 & -121.969487 & 577355 & 5280516 \\
\hline 2588 & 612 & 47.673366 & -121.968150 & 577456 & 5280513 \\
\hline 2589 & 7461 & 47.673371 & -121.966791 & 577558 & 5280515 \\
\hline 2590 & 508 & 47.673356 & -121.965467 & 577657 & 5280514 \\
\hline 2591 & A109 & 47.673343 & -121.964137 & 577757 & 5280514 \\
\hline 2592 & 509 & 47.675475 & -121.962812 & 577853 & 5280753 \\
\hline 2593 & 7600 & 47.677692 & -121.961504 & 577948 & 5281000 \\
\hline 2594 & 613 & 47.678584 & -121.959813 & 578074 & 5281101 \\
\hline 2595 & 504 & 47.678892 & -121.958527 & 578170 & 5281137 \\
\hline 2596 & A110 & 47.678668 & -121.957190 & 578270 & 5281113 \\
\hline 2597 & 7327 & 47.678363 & -121.955863 & 578370 & 5281081 \\
\hline 2598 & A111 & 47.677919 & -121.954704 & 578458 & 5281032 \\
\hline 2599 & 614 & 47.677071 & -121.953213 & 578571 & 5280940 \\
\hline 2600 & 505 & 47.676156 & -121.952416 & 578632 & 5280839 \\
\hline 2601 & 501 & 47.675226 & -121.950940 & 578745 & 5280737 \\
\hline 2602 & 506 & 47.674433 & -121.949991 & 578817 & 5280650 \\
\hline 2603 & A096 & 47.673664 & -121.948697 & 578915 & 5280566 \\
\hline
\end{tabular}




\begin{tabular}{|c|c|c|c|c|c|}
\hline 2604 & A095 & 47.672480 & -121.947022 & 579043 & 5280436 \\
\hline 2605 & 7341 & 47.671891 & -121.945686 & 579144 & 5280372 \\
\hline 2606 & 615 & 47.671533 & -121.944429 & 579239 & 5280333 \\
\hline 2607 & A094 & 47.671740 & -121.943071 & 579341 & 5280358 \\
\hline 2608 & 502 & 47.671957 & -121.941758 & 579439 & 5280383 \\
\hline 2609 & $\bullet 7622$ & 47.672202 & -121.940457 & 579536 & 5280412 \\
\hline 2610 & 617 & 47.672043 & -121.939146 & 579635 & 5280395 \\
\hline 2611 & 503 & 47.671813 & -121.937774 & 579738 & 5280371 \\
\hline 2612 & A081 & 47.671832 & -121.936691 & 579819 & 5280374 \\
\hline 2613 & 7297 & 47.671808 & -121.935336 & 579921 & 5280373 \\
\hline 2614 & 535 & 47.671804 & -121.933984 & 580023 & 5280374 \\
\hline 2615 & 618 & 47.671778 & -121.932660 & 580122 & 5280373 \\
\hline 2616 & A161 & 47.671751 & -121.931299 & 580224 & 5280371 \\
\hline 2617 & 7326 & 47.671454 & -121.930061 & 580318 & 5280339 \\
\hline 2618 & A091 & 47.670306 & -121.928781 & 580415 & 5280213 \\
\hline 2619 & 533 & 47.668459 & -121.927638 & 580504 & 5280009 \\
\hline 2620 & 619 & 47.666032 & -121.925512 & 580667 & 5279741 \\
\hline 2621 & 7275 & 47.665716 & -121.924361 & 580754 & 5279707 \\
\hline 2622 & 532 & 47.665365 & -121.923458 & 580823 & 5279669 \\
\hline 2623 & 534 & 47.664970 & -121.922059 & 580928 & 5279627 \\
\hline 2624 & A092 & 47.664612 & -121.920714 & 581030 & 5279589 \\
\hline 2625 & 7063 & 47.664238 & -121.919348 & 581133 & 5279548 \\
\hline 2626 & 620 & 47.663873 & -121.917974 & 581237 & 5279509 \\
\hline 2627 & 530 & 47.663492 & -121.916631 & 581338 & 5279468 \\
\hline 2628 & 529 & 47.663146 & -121.915252 & 581442 & 5279431 \\
\hline 2629 & 531 & 47.662773 & -121.913891 & 581545 & 5279391 \\
\hline 2630 & 526 & 47.662485 & -121.912759 & 581630 & 5279360 \\
\hline 2631 & 621 & 47.662313 & -121.911047 & 581759 & 5279343 \\
\hline 2632 & A122 & 47.659960 & -121.910095 & 581834 & 5279083 \\
\hline 2633 & 7039 & 47.658847 & -121.909164 & 581906 & 5278960 \\
\hline 2634 & A163 & 47.658843 & -121.907792 & 582009 & 5278961 \\
\hline 2635 & 537 & 47.658764 & -121.906501 & 582106 & 5278954 \\
\hline 2636 & 622 & 47.658770 & -121.905138 & 582208 & 5278956 \\
\hline 2637 & 6044 & 47.658744 & -121.903793 & 582309 & 5278954 \\
\hline 2638 & 536 & 47.658772 & -121.902469 & 582409 & 5278959 \\
\hline 2639 & A162 & 47.658776 & -121.901242 & 582501 & 5278960 \\
\hline 2640 & 623 & 47.658671 & -121.899814 & 582608 & 5278950 \\
\hline 2641 & 6053 & 47.658602 & -121.898534 & 582705 & 5278944 \\
\hline 2642 & 624 & 47.658569 & -121.897234 & 582802 & 5278942 \\
\hline 2643 & 528 & 47.660254 & -121.895340 & 582942 & 5279131 \\
\hline 2644 & A181 & 47.662018 & -121.894255 & 583020 & 5279328 \\
\hline 2645 & $\bullet 7612$ & 47.661531 & -121.892645 & 583142 & 5279276 \\
\hline 2646 & 527 & 47.659843 & -121.891621 & 583222 & 5279089 \\
\hline 2647 & 593 & 47.659111 & -121.889867 & 583355 & 5279010 \\
\hline 2648 & A183 & 47.659585 & -121.888343 & 583468 & 5279064 \\
\hline
\end{tabular}

\begin{tabular}{|c|c|c|c|c|c|c|}
\hline 2657 & 538 & 47.647934 & -121.882707 & 583910 & 5277775 & 56 \\
\hline 2663 & 605 & 47.651471 & -121.868223 & 584992 & 5278184 & 128 \\
\hline 2664 & 604 & 47.652329 & -121.867514 & 585044 & 5278280 & 139 \\
\hline 2665 & 598 & 47.653125 & -121.866097 & 585149 & 5278370 & 137 \\
\hline 2666 & 597 & 47.653768 & -121.864726 & 585251 & 5278443 & 131 \\
\hline 2667 & 596 & 47.654282 & -121.863272 & 585359 & 5278502 & 129 \\
\hline 2668 & 595 & 47.654881 & -121.862024 & 585452 & 5278570 & 148 \\
\hline 2669 & 594 & 47.655762 & -121.860669 & 585552 & 5278669 & \\
\hline 2670 & A178 & 47.655770 & -121.859452 & 585644 & 5278672 & 202 \\
\hline 2671 & A124 & 47.654197 & -121.857890 & 585764 & 5278499 & \\
\hline 2672 & A180 & 47.651282 & -121.856713 & 585857 & 5278176 & 197 \\
\hline 2673 & A123 & 47.650742 & -121.855172 & 585973 & 5278118 & 172 \\
\hline 2674 & A188 & 47.650284 & -121.853616 & 586091 & 5278068 & 160 \\
\hline 2675 & A187 & 47.649870 & -121.852300 & 586190 & 5278024 & 159 \\
\hline 2676 & A172 & 47.648977 & -121.851194 & 586275 & 5277926 & 168 \\
\hline 2677 & A171 & 47.648118 & -121.849917 & 586372 & 5277832 & 181 \\
\hline 2678 & 7047 & 47.653340 & -121.848626 & 586461 & 5278414 & 183 \\
\hline 2679 & 566 & 47.653525 & -121.847205 & 586567 & 5278436 & 182 \\
\hline 2680 & 7081 & 47.655000 & -121.845771 & 586672 & 5278601 & 192 \\
\hline 2681 & 7038 & 47.655773 & -121.844283 & 586783 & 5278689 & 201 \\
\hline 2682 & 567 & 47.656038 & -121.842940 & 586883 & 5278720 & 207 \\
\hline 2683 & A168 & 47.656342 & -121.841525 & 586989 & 5278755 & 203 \\
\hline 2684 & 568 & 47.656596 & -121.840110 & 587095 & 5278785 & 196 \\
\hline 2685 & 7098 & 47.656841 & -121.838817 & 587191 & 5278814 & 204 \\
\hline 2686 & 7088 & 47.657022 & -121.837514 & 587289 & 5278835 & 193 \\
\hline 2687 & 7082 & 47.657019 & -121.836258 & 587383 & 5278836 & 193 \\
\hline 2688 & A166 & 47.657250 & -121.835230 & 587460 & 5278863 & 194 \\
\hline 2689 & $\bullet 7597$ & 47.657022 & -121.833829 & 587566 & 5278839 & 206 \\
\hline 2690 & 569 & 47.656418 & -121.832669 & 587654 & 5278774 & 214 \\
\hline 2691 & 7071 & 47.656234 & -121.831359 & 587752 & 5278755 & 210 \\
\hline 2692 & 6111 & 47.656668 & -121.829996 & 587854 & 5278804 & 208 \\
\hline 2693 & 6087 & 47.657113 & -121.828651 & 587954 & 5278855 & 201 \\
\hline 2694 & A165 & 47.657187 & -121.827331 & 588053 & 5278865 & 186 \\
\hline 2695 & $* 607$ & 47.657377 & -121.826011 & 588152 & 5278888 & 177 \\
\hline 2696 & $* 608$ & 47.657025 & -121.824710 & 588250 & 5278850 & 180 \\
\hline 2697 & 6060 & 47.656213 & -121.823272 & 588360 & 5278762 & 178 \\
\hline 2698 & 570 & 47.657679 & -121.821953 & 588456 & 5278926 & 167 \\
\hline 2699 & A164 & 47.658198 & -121.820481 & 588566 & 5278985 & 197 \\
\hline 2700 & 571 & 47.657857 & -121.819190 & 588663 & 5278949 & 184 \\
\hline 2701 & 6107 & 47.658426 & -121.817573 & 588784 & 5279014 & 189 \\
\hline 2702 & $* 609$ & 47.659135 & -121.816442 & 588868 & 5279094 & 203 \\
\hline 2703 & 572 & 47.659837 & -121.815298 & 588952 & 5279173 & 202 \\
\hline 2704 & 6039 & 47.661242 & -121.813890 & 589056 & 5279331 & 193 \\
\hline 2705 & A177 & 47.660156 & -121.812291 & 589177 & 5279212 & 221 \\
\hline 2706 & A175 & 47.657341 & -121.810796 & 589295 & 5278901 & 248 \\
\hline
\end{tabular}




\begin{tabular}{|c|c|c|c|c|c|}
\hline 2709 & 573 & 47.655458 & -121.808745 & 589452 & 5278694 \\
\hline 2710 & 574 & 47.657140 & -121.806679 & 589604 & 5278884 \\
\hline 2711 & 576 & 47.660330 & -121.805165 & 589712 & 5279240 \\
\hline 2712 & 577 & 47.658795 & -121.803742 & 589822 & 5279071 \\
\hline 2714 & 578 & 47.658077 & -121.801181 & 590015 & 5278994 \\
\hline 2715 & 579 & 47.660226 & -121.799334 & 590150 & 5279235 \\
\hline 2716 & 580 & 47.659817 & -121.798151 & 590240 & 5279191 \\
\hline 2717 & 581 & 47.659094 & -121.796820 & 590341 & 5279112 \\
\hline 2718 & A174 & 47.656990 & -121.794637 & 590508 & 5278881 \\
\hline 2719 & 6088 & 47.656850 & -121.793610 & 590586 & 5278867 \\
\hline 2720 & 6041 & 47.657462 & -121.792525 & 590666 & 5278936 \\
\hline 2721 & $* 610$ & 47.657128 & -121.791456 & 590747 & 5278900 \\
\hline 2722 & 582 & 47.656159 & -121.789071 & 590928 & 5278795 \\
\hline 2723 & A173 & 47.654967 & -121.788038 & 591007 & 5278664 \\
\hline 2724 & 6093 & 47.652522 & -121.787032 & 591087 & 5278393 \\
\hline 2725 & 583 & 47.654276 & -121.785226 & 591220 & 5278590 \\
\hline 2726 & $* 611$ & 47.653614 & -121.783857 & 591324 & 5278518 \\
\hline 2727 & A186 & 47.651723 & -121.782602 & 591421 & 5278310 \\
\hline 2728 & A185 & 47.649945 & -121.781396 & 591515 & 5278114 \\
\hline 2729 & $\bullet 7299$ & 47.650062 & -121.779661 & 591645 & 5278129 \\
\hline 2730 & $* 612$ & 47.650760 & -121.778314 & 591745 & 5278208 \\
\hline 2731 & $* 613$ & 47.652190 & -121.777329 & 591816 & 5278368 \\
\hline 2732 & 6124 & 47.653961 & -121.776127 & 591904 & 5278566 \\
\hline 2733 & 585 & 47.653751 & -121.774808 & 592003 & 5278544 \\
\hline 2734 & A169 & 47.653399 & -121.773420 & 592108 & 5278507 \\
\hline 2735 & 586 & 47.653656 & -121.7 & 592233 & 5278537 \\
\hline 2736 & 6083 & 47.645152 & -121.773442 & 592121 & 5277590 \\
\hline 2737 & 6084 & 47.648165 & -121.771004 & 592299 & 5277928 \\
\hline 2738 & 6097 & 47.651319 & -121.769000 & 592443 & 5278281 \\
\hline 2743 & A022 & 47.651750 & -121.761676 & 592993 & 5278338 \\
\hline 2744 & 6004 & 47.653103 & -121.759716 & 593137 & 5278490 \\
\hline 2745 & 587 & 47.655185 & -121.759213 & 593172 & 5278722 \\
\hline 2746 & $* 614$ & 47.657986 & -121.757789 & 593273 & 5279035 \\
\hline 2747 & 6132 & 47.656163 & -121.756555 & 593369 & 5278834 \\
\hline 2748 & 6069 & 47.655884 & -121.755298 & 593464 & 5278805 \\
\hline 2749 & 591 & 47.655527 & -121.754009 & 593562 & 5278767 \\
\hline 2750 & A010 & 47.653845 & -121.752976 & 593642 & 5278581 \\
\hline 2751 & A009 & 47.654176 & -121.752070 & 593710 & 5278619 \\
\hline 2752 & A017 & 47.653581 & -121.750918 & 593797 & 5278554 \\
\hline 2753 & $* 615$ & 47.656970 & -121.748365 & 593983 & 5278934 \\
\hline 2754 & 6037 & 47.654614 & -121.747091 & 594083 & 5278674 \\
\hline 2755 & 588 & 47.654078 & -121.745678 & 594190 & 5278616 \\
\hline 2756 & 6021 & 47.654172 & -121.744319 & 594292 & 5278628 \\
\hline 2757 & $* 616$ & 47.654034 & -121.742940 & 594396 & 5278614 \\
\hline 2758 & 589 & 47.653399 & -121.741685 & 594491 & 5278545 \\
\hline
\end{tabular}

\begin{tabular}{|c|c|c|c|c|c|c|}
\hline 2759 & A012 & 47.651757 & -121.740785 & 594561 & 5278364 & 378 \\
\hline 2760 & 6001 & 47.650874 & -121.739156 & 594685 & 5278268 & 379 \\
\hline 2761 & A011 & 47.650322 & -121.737701 & 594796 & 5278208 & 377 \\
\hline 2762 & $* 617$ & 47.650075 & -121.736247 & 594905 & 5278182 & 381 \\
\hline 2763 & 590 & 47.650130 & -121.734911 & 595006 & 5278190 & 391 \\
\hline 2764 & 6090 & 47.650222 & -121.733582 & 595105 & 5278202 & 391 \\
\hline 2765 & A019 & 47.650223 & -121.732231 & 595207 & 5278204 & 393 \\
\hline 2766 & 592 & 47.650106 & -121.730934 & 595304 & 5278192 & 400 \\
\hline 2767 & $* 618$ & 47.650059 & -121.729534 & 595409 & 5278189 & 407 \\
\hline 2768 & $\bullet 7278$ & 47.649907 & -121.728250 & 595506 & 5278173 & 413 \\
\hline 2769 & 551 & 47.649545 & -121.726997 & 595601 & 5278135 & 433 \\
\hline 2770 & A020 & 47.649120 & -121.725884 & 595685 & 5278089 & 427 \\
\hline 2771 & *619 & 47.648515 & -121.724746 & 595772 & 5278023 & 439 \\
\hline 2772 & 6103 & 47.647830 & -121.723819 & 595843 & 5277948 & 435 \\
\hline 2773 & A021 & 47.647838 & -121.722631 & 595932 & 5277950 & 445 \\
\hline 2774 & 552 & 47.648508 & -121.721288 & 596032 & 5278027 & 463 \\
\hline 2775 & A005 & 47.652054 & -121.718763 & 596215 & 5278424 & 387 \\
\hline 2776 & 6085 & 47.651251 & -121.717872 & 596283 & 5278336 & 416 \\
\hline 2777 & $* 620$ & 47.651576 & -121.716490 & 596386 & 5278373 & 389 \\
\hline 2778 & 6114 & 47.651935 & -121.715299 & 596475 & 5278415 & 404 \\
\hline 2779 & A006 & 47.652242 & -121.713967 & 596574 & 5278451 & 393 \\
\hline 2780 & 6127 & 47.652574 & -121.712484 & 596685 & 5278489 & 399 \\
\hline 2782 & 553 & 47.652628 & -121.709770 & 596889 & 5278499 & 410 \\
\hline 2784 & $* 621$ & 47.652243 & -121.707183 & 597084 & 5278459 & 442 \\
\hline 2786 & 554 & 47.652234 & -121.704542 & 597282 & 5278462 & 457 \\
\hline 2788 & 6018 & 47.652821 & -121.701996 & 597472 & 5278530 & 469 \\
\hline 2790 & A007 & 47.652071 & -121.699236 & 597681 & 5278450 & 491 \\
\hline 2792 & 6148 & 47.650604 & -121.696718 & 597873 & 5278290 & 523 \\
\hline 2794 & *622 & 47.649603 & -121.694204 & 598063 & 5278182 & 551 \\
\hline 2796 & 6024 & 47.648860 & -121.691735 & 598250 & 5278103 & 567 \\
\hline 2798 & 555 & 47.646930 & -121.688667 & 598484 & 5277892 & 607 \\
\hline 2800 & A008 & 47.645337 & -121.686108 & 598680 & 5277718 & 592 \\
\hline 2802 & 556 & 47.649339 & -121.682661 & 598931 & 5278167 & 559 \\
\hline 2804 & 6026 & 47.649702 & -121.679566 & 599163 & 5278212 & 552 \\
\hline 2806 & $* 623$ & 47.649322 & -121.677042 & 599353 & 5278173 & 539 \\
\hline 2808 & $\bullet 7606$ & 47.650020 & -121.674419 & 599549 & 5278254 & 531 \\
\hline 2810 & A001 & 47.651144 & -121.672067 & 599723 & 5278382 & 522 \\
\hline 2812 & 6031 & 47.644887 & -121.670108 & 599882 & 5277689 & 469 \\
\hline 2814 & 557 & 47.645826 & -121.667434 & 600081 & 5277797 & 487 \\
\hline 2816 & $* 624$ & 47.648105 & -121.664725 & 600280 & 5278053 & 471 \\
\hline 2818 & A014 & 47.648906 & -121.662096 & 600476 & 5278146 & 487 \\
\hline 2820 & 6058 & 47.648648 & -121.659483 & 600673 & 5278121 & 500 \\
\hline 2822 & $\mathrm{~A} 002$ & 47.648704 & -121.656771 & 600876 & 5278130 & 484 \\
\hline 2824 & 558 & 47.649133 & -121.653914 & 601090 & 5278182 & 499 \\
\hline 2826 & $* 625$ & 47.649317 & -121.651467 & 601273 & 5278205 & 492 \\
\hline
\end{tabular}




\begin{tabular}{|c|c|c|c|c|c|c|}
\hline 2828 & 6064 & 47.649740 & -121.648916 & 601464 & 5278256 & 475 \\
\hline 2830 & 559 & 47.649525 & -121.646096 & 601676 & 5278235 & 472 \\
\hline 2832 & A003 & 47.652893 & -121.643773 & 601844 & 5278613 & 461 \\
\hline 2834 & $* 633$ & 47.654633 & -121.641428 & 602017 & 5278809 & 448 \\
\hline 2836 & 6102 & 47.655811 & -121.637942 & 602277 & 5278945 & 467 \\
\hline 2838 & A004 & 47.656472 & -121.635042 & 602493 & 5279022 & 474 \\
\hline 2840 & 6049 & 47.657277 & -121.632201 & 602705 & 5279115 & 472 \\
\hline 2842 & A016 & 47.658218 & -121.630134 & 602858 & 5279223 & 472 \\
\hline 2844 & 6120 & 47.659352 & -121.627383 & 603062 & 5279352 & 473 \\
\hline 2846 & 560 & 47.659900 & -121.624924 & 603246 & 5279416 & 479 \\
\hline 2848 & $* 627$ & 47.659888 & -121.622236 & 603448 & 5279419 & 473 \\
\hline 2850 & 561 & 47.660349 & -121.619462 & & 5279474 & 472 \\
\hline 2852 & $\bullet 7295$ & 47.660831 & -121.616803 & 603854 & 5279531 & 473 \\
\hline 2854 & A015 & 47.661508 & -121.614231 & 604046 & 5279610 & 483 \\
\hline 2856 & 6091 & 47.6 & -121 . & & 9580 & 488 \\
\hline 2858 & $* 628$ & 47.660963 & -121.608646 & 604466 & 5279556 & 475 \\
\hline 2860 & 6022 & 47.661748 & -121.606206 & 604648 & 5279647 & 480 \\
\hline 2862 & 562 & 47.6 & -121 & 883 & 9714 & 494 \\
\hline 2864 & $* 629$ & 47.6 & -121 & 060 & 9775 & 481 \\
\hline 2866 & $* 630$ & 47.662558 & -121.5 & 241 & 5279748 & 479 \\
\hline 2868 & 6098 & 47.6 & -121. & 605437 & 5279718 & 477 \\
\hline 2870 & $* 631$ & 47.6 & -121 & & 752 & 495 \\
\hline 2872 & 563 & 47.6 & -121. & & 9643 & 487 \\
\hline • 2874 & 6067 & 47.6 & -121. & 046 & 5279643 & 475 \\
\hline 2876 & $* 647$ & 47.660204 & -121. & 606249 & 5279504 & 479 \\
\hline 3000 & $* 568$ & 47. & -122 & 952 & 0932 & 9 \\
\hline 3010 & 633 & 47.761683 & -122.562165 & & 5289906 & 19 \\
\hline 3020 & $\bullet 7270$ & 47.751751 & -122.562810 & 532769 & 5288802 & 27 \\
\hline 3030 & A147 & 47.7 & -122 & 533188 & 7970 & 41 \\
\hline 3060 & 632 & 47.7 & -122.5 & 532708 & 5284983 & 20 \\
\hline 3070 & 7272 & 47.7 & -122.5 & & 3861 & 89 \\
\hline 3080 & A 146 & 47.698554 & -122.556450 & 533279 & 5282892 & 58 \\
\hline 3090 & 186 & 47.688561 & -122.5 & 533839 & 5281784 & 40 \\
\hline 3100 & $* 567$ & 47.679835 & -122.547586 & 533956 & 5280815 & 37 \\
\hline 3110 & 7041 & 47.672146 & -122.545819 & 534094 & 5279962 & 37 \\
\hline 3130 & A145 & 47.652648 & -122.547791 & 533959 & 5277794 & 98 \\
\hline 3140 & 185 & 47.644169 & -122.547732 & 533968 & 5276851 & 81 \\
\hline 3150 & A144 & 47.637097 & -122.542416 & 534372 & 5276068 & 57 \\
\hline 3160 & $* 565$ & 47.626707 & -122.539348 & 534610 & 5274914 & \\
\hline 3170 & 184 & 47.618284 & -122.540878 & 534500 & 5273978 & 47 \\
\hline 3180 & A143 & 47.609079 & -122.537934 & 534728 & 5272956 & 86 \\
\hline 3190 & 182 & 47.600762 & -122.536062 & 534874 & 5272032 & 58 \\
\hline 3200 & A 142 & 47.590285 & -122.532582 & 535142 & 5270870 & 17 \\
\hline 3210 & 181 & 47.581409 & -122.523350 & 535843 & 5269887 & 51 \\
\hline 4010 & $* 594$ & 47.769787 & -122.279394 & 553993 & 5290965 & 159 \\
\hline
\end{tabular}

\begin{tabular}{|c|c|c|c|c|c|c|}
\hline 4020 & •7628 & 47.760269 & -122.283096 & 553725 & 5289905 & 48 \\
\hline 4030 & 413 & 47.751691 & -122.283011 & 553740 & 5288951 & 27 \\
\hline 4040 & A141 & 47.744134 & -122.285645 & 553550 & 5288110 & \\
\hline 4050 & 410 & 47.733745 & -122.285617 & 553563 & 5286955 & \\
\hline 4060 & $* 595$ & 47.725890 & -122.286187 & 553529 & 5286081 & \\
\hline 4070 & 7062 & 47.715949 & -122.284378 & 553674 & 5284978 & \\
\hline 4080 & 411 & 47.706263 & -122.277835 & 554175 & 5283906 & \\
\hline 4090 & A133 & 47.697771 & -122.277982 & 554173 & 5282962 & \\
\hline 4100 & 412 & 47.689782 & -122.266566 & 555038 & 5282082 & \\
\hline 4120 & $* 593$ & 47.670141 & -122.263580 & 555283 & 5279901 & 57 \\
\hline 4130 & 409 & 47.661560 & -122.268960 & 554888 & 5278944 & \\
\hline 4140 & A160 & 47.653243 & -122.277716 & 554239 & 5278013 & \\
\hline 4150 & 7096 & 47.642621 & -122.283589 & 553809 & 5276829 & \\
\hline 4160 & 7054 & 47.634141 & -122.277663 & 554263 & 5275891 & \\
\hline 4170 & *596 & 47.624242 & -122.284633 & 553749 & 5274786 & \\
\hline 4180 & 403 & 47.617674 & -122.280780 & 554046 & 5274058 & \\
\hline 4190 & •7608 & 47.607015 & -122.283886 & 553823 & 5272871 & \\
\hline 4200 & *597 & 47.598029 & -122.287299 & 553576 & 5271870 & 23 \\
\hline 4210 & 425 & 47.577076 & -122.283127 & 553911 & 5269545 & \\
\hline 4220 & A088 & 47.577076 & -122.283092 & 553914 & 5269545 & 14 \\
\hline 4230 & 424 & 47.570580 & -122.280101 & 554145 & 5268825 & 18 \\
\hline 4240 & 423 & 47.562719 & -122.268274 & 555043 & 5267960 & 15 \\
\hline 4250 & A089 & 47.551721 & -122.257064 & 555898 & 5266745 & \\
\hline 5000 & $\bullet 7284$ & 47.743521 & -122.115097 & 566336 & 5288173 & 168 \\
\hline 5007 & A090 & 47.737830 & -122.090310 & 568202 & 5287562 & 56 \\
\hline 5010 & 475 & 47.733483 & -122.115281 & 566335 & 5287058 & 156 \\
\hline 5020 & A083 & 47.724798 & -122.115530 & 566327 & 5286092 & 111 \\
\hline 5030 & 474 & 47.716496 & -122.110415 & 566722 & 5285174 & 95 \\
\hline 5040 & A082 & 47.706801 & -122.110743 & 566709 & 5284096 & 97 \\
\hline 5050 & 400 & 47.698347 & -122.111830 & 566639 & 5283156 & 89 \\
\hline 5060 & 473 & 47.689297 & -122.111918 & 566643 & 5282150 & 114 \\
\hline 5070 & 631 & 47.679995 & -122.112257 & 566630 & 5281116 & 97 \\
\hline 5080 & 472 & 47.672674 & -122.109195 & 566869 & 5280305 & 14 \\
\hline 5100 & A087 & 47.650098 & -122.113634 & 566565 & 5277792 & 24 \\
\hline 5110 & 467 & 47.643675 & -122.110954 & 566774 & 5277080 & 49 \\
\hline 5120 & 7442 & 47.633964 & -122.110881 & 566792 & 5276001 & 87 \\
\hline 5130 & 628 & 47.626225 & -122.111714 & 566739 & 5275140 & 121 \\
\hline 5140 & 468 & 47.616819 & -122.109937 & 566885 & 5274096 & 99 \\
\hline 5150 & A086 & 47.606878 & -122.113345 & 566641 & 5272989 & 25 \\
\hline 5160 & 469 & 47.598921 & -122.110714 & 566849 & 5272107 & 42 \\
\hline 5170 & A085 & 47.588874 & -122.111797 & 566780 & 5270989 & \\
\hline 5180 & 470 & 47.580880 & -122.112732 & 566720 & 5270100 & \\
\hline 5190 & 471 & 47.572253 & -122.112679 & 566735 & 5269141 & 77 \\
\hline 5200 & A084 & 47.563102 & -122.115117 & 566563 & 5268122 & 209 \\
\hline 5210 & $\bullet 7617$ & 47.548523 & -122.115515 & 566552 & 5266501 & 346 \\
\hline
\end{tabular}




\begin{tabular}{rrrrrrr}
6000 & 499 & 47.772532 & -122.036566 & 572183 & 5291468 & 160 \\
6010 & 545 & 47.765382 & -122.037146 & 572149 & 5290673 & 167 \\
6020 & $\mathrm{~A} 148$ & 47.754949 & -122.033128 & 572465 & 5289517 & 183 \\
6030 & 541 & 47.745169 & -122.029438 & 572755 & 5288434 & 163 \\
6040 & $\bullet 7614$ & 47.735483 & -122.028715 & 572823 & 5287358 & 173 \\
6050 & 544 & 47.726627 & -122.035069 & 572359 & 5286368 & 144 \\
6060 & 610 & 47.718595 & -122.034551 & 572409 & 5285475 & 144 \\
6070 & 7057 & 47.709162 & -122.027062 & 572983 & 5284434 & 192 \\
6080 & $\mathrm{~A} 093$ & 47.701041 & -122.024352 & 573198 & 5283534 & 172 \\
6110 & 609 & 47.672486 & -122.032888 & 572597 & 5280352 & 167 \\
6120 & 547 & 47.663192 & -122.034009 & 572526 & 5279318 & 161 \\
6130 & 540 & 47.654609 & -122.035262 & 572444 & 5278363 & 89 \\
6140 & 7094 & 47.645970 & -122.035318 & 572452 & 5277403 & 53 \\
6150 & $\mathrm{~A} 080$ & 47.635009 & -122.037361 & 572313 & 5276183 & 124 \\
6160 & 543 & 47.627059 & -122.035524 & 572462 & 5275301 & 127 \\
6170 & $\mathrm{~A} 079$ & 47.618507 & -122.035432 & 572481 & 5274351 & 131 \\
6180 & $\bullet 7340$ & 47.610177 & -122.035534 & 572485 & 5273425 & 113 \\
6190 & 546 & 47.601140 & -122.035578 & 572494 & 5272421 & 165 \\
6200 & $\mathrm{~A} 078$ & 47.591894 & -122.035579 & 572507 & 5271393 & 137 \\
6210 & $\bullet 7605$ & 47.581374 & -122.035659 & 572515 & 5270224 & 138 \\
6220 & 542 & 47.574246 & -122.035839 & 572512 & 5269432 & 123 \\
6230 & 607 & 47.573470 & -122.036040 & 572497 & 5269345 & 126 \\
6240 & 539 & 47.559137 & -122.035911 & 572527 & 5267752 & 153 \\
\hline Station location & determined from a digital USGS topographic map.
\end{tabular}

-Continuously recording REFTEK Model 07.

$*$ SGR-III

Notes: Unit numbers Axxx correspond to PRS-1's; Oxxx correspond to PRS-4's. Unit numbers 6001-6148 correspond to REFTEK Model O6's.

Unit numbers 7038-7629 correspond to REFTEK Model 07's.

Unit numbers 1-660 are Texans.

SGR units at Stations 1000, 1084, 1103, 1114, 2024, 2295, 2359, 2695, 2870,

4010 and 4120 failed to record useful data. SGR Station 2878 was not deployed.

PRS 9900 was located at the Kitsap County Fairgrounds, near the Presidents Hall (at Station 2057).

OBS A4 (Station 2202) was not recovered. OBS A8 (Station 2232) did not

record data. OBS D4 (Station 2220) accidentally released prematurely during the experiment between shot 24 and 25 .

Note: The UTM coordinates in the tape headers for the OBS's (stations 2190 to 2232) are incorrect. Use the UTM coordinates from Table 2. 
Table 3. Shot list ordered chronologically by shot time.

\begin{tabular}{|c|c|c|c|c|c|c|c|c|c|c|c|}
\hline $\begin{array}{l}\text { Shot } \\
\text { No. }\end{array}$ & $\begin{array}{c}\text { Shot } \\
\text { Point } \\
\text { No. }\end{array}$ & $\begin{array}{c}\text { Shot Time } \\
\text { (JD:Hr:Mn:S) } \\
\text { UTC }\end{array}$ & $\begin{array}{c}\text { Shot } \\
\text { Point } \\
\text { Latitude }\end{array}$ & $\begin{array}{c}\text { Shot } \\
\text { Point } \\
\text { Longitude }\end{array}$ & $\begin{array}{c}\text { UTM } \\
\text { Easting } \\
(\mathrm{m})\end{array}$ & $\begin{array}{c}\text { UTM } \\
\text { Northing } \\
\text { (m) }\end{array}$ & $\begin{array}{l}\text { Shot } \\
\text { Elev. } \\
(\mathrm{m})\end{array}$ & $\begin{array}{l}\text { Shot } \\
\text { Depth } \\
(\mathrm{m})\end{array}$ & $\begin{array}{c}\text { Trace } \\
\text { Header } \\
\text { Stat. }\end{array}$ & $\begin{array}{l}\text { Shot } \\
\text { Size } \\
(\mathrm{lbs})\end{array}$ & $\begin{array}{l}\text { Shot } \\
\text { Size } \\
(\mathrm{kgs})\end{array}$ \\
\hline 1 & $\overline{\text { SP02 }}$ & 263:08:00:00 & 47.741223 & -123.056398 & 495772 & 5287540 & 155 & 23 & 20 & 250 & 113 \\
\hline 2 & SP06 & 263:08:02:00 & 47.707970 & -122.892340 & 508076 & 5283849 & 5 & 15 & 60 & 50 & 23 \\
\hline 3 & SP30 & 263:08:04:00 & 47.660151 & -121.812390 & 589170 & 5279212 & 224 & 23 & 300 & 250 & 113 \\
\hline 4 & SP31 & 263:08:06:00 & 47.655139 & -121.758625 & 593216 & 5278718 & 355 & 23 & 310 & 250 & 113 \\
\hline 5 & SP34 & 263:08:08:00 & 47.653562 & -121.642726 & 601922 & 5278689 & 467 & 23 & 340 & 250 & 113 \\
\hline 6 & SP01 & 263:09:30:00 & 47.729520 & -123.086529 & 493512 & 5286242 & 238 & 37 & 10 & 2800 & 1267 \\
\hline 7 & SP05 & $263: 09: 32: 00$ & 47.730716 & -122.947215 & 503958 & 5286372 & 414 & 30 & 51 & 2000 & 905 \\
\hline 8 & SP29 & 263:09:34:00 & 47.657893 & -121.860752 & 585543 & 5278906 & 129 & 27 & 291 & 800 & 362 \\
\hline 9 & SP32 & 263:09:36:00 & 47.651181 & -121.717902 & 596281 & 5278328 & 389 & 30 & 320 & 2000 & 905 \\
\hline 10 & SP35 & $263: 11: 08: 00$ & 47.660939 & -121.616558 & 603872 & 5279543 & 468 & 27 & 350 & 2400 & 1086 \\
\hline 11 & SP04 & 264:08:00:00 & 47.716137 & -122.991552 & 500634 & 5284751 & 136 & 23 & 40 & 250 & 113 \\
\hline 12 & SP09 & 264:08:02:00 & 47.693580 & -122.779130 & 516573 & 5282267 & 86 & 15 & 90 & 50 & 23 \\
\hline 13 & SP33 & 264:08:06:00 & 47.652125 & -121.672736 & 599671 & 5278490 & 526 & 23 & 330 & 250 & 113 \\
\hline 14 & SP10 & 264:08:10:00 & 47.699421 & -122.724739 & 520652 & 5282930 & 121 & 23 & 100 & 150 & 68 \\
\hline 15 & SP11 & 264:08:12:00 & 47.680029 & -122.718209 & 521150 & 5280776 & 117 & 24 & 111 & 500 & 226 \\
\hline 16 & SP24 & 264:08:14:00 & 47.682420 & -122.022710 & 573347 & 5281466 & 171 & 24 & 240 & 500 & 226 \\
\hline 17 & SP01 & 264:09:30:00 & 47.729520 & -123.086529 & 493512 & 5286242 & 238 & 37 & 10 & 2800 & 1267 \\
\hline 18 & SP08 & $264: 09: 3$ & 47.705836 & -122.801583 & 514885 & 5283625 & 44 & 23 & 80 & 250 & 113 \\
\hline 19 & SP05 & 264:09:34:00 & 47.729511 & -122.945819 & 504063 & 5286239 & 404 & 30 & 52 & 2000 & 905 \\
\hline 20 & SP32 & $264: 09: 36: 00$ & 47.651181 & -121.717902 & 596281 & 5278328 & 389 & 30 & 320 & 2000 & 905 \\
\hline 21 & SP12 & 264:09:38:00 & 47.675732 & -122.740495 & 519479 & 5280293 & 84 & 24 & 120 & 500 & 226 \\
\hline 22 & SP11 & $264: 09: 42: 00$ & 47.679773 & -122.718313 & 521142 & 5280748 & 117 & 24 & 112 & 500 & 226 \\
\hline 23 & SP24 & 264:09:44:00 & 47.682420 & -122.022710 & 573347 & 5281466 & 171 & 24 & 240 & 500 & 226 \\
\hline 24 & SP12 & $264: 11: 08: 00$ & 47.675732 & -122.740495 & 519479 & 5280293 & 84 & 24 & 120 & 500 & 226 \\
\hline 25 & SP21 & 265:08:00:00 & 47.682904 & -122.249409 & 556333 & 5281330 & 17 & 24 & 211 & 400 & 181 \\
\hline 26 & SP27 & 265:08:04:00 & 47.672020 & -121.930550 & 580280 & 5280402 & 9 & 23 & 270 & 250 & 113 \\
\hline 27 & SP26 & 265:08:06:00 & 47.644160 & -121.946420 & 579131 & 5277289 & 158 & 21 & 260 & 500 & 226 \\
\hline 28 & SP14 & 265:08:08:00 & 47.677027 & -122.631557 & 527655 & 5280470 & 73 & 18 & 140 & 50 & 23 \\
\hline 29 & SP15 & 265:08:10:00 & 47.661350 & -122.578149 & 531674 & 5278748 & 45 & 18 & 150 & 50 & 23 \\
\hline 30 & SP19 & $265: 08: 12: 00$ & 47.668744 & -122.345883 & 549106 & 5279691 & 79 & 18 & 190 & 25 & 11 \\
\hline 31 & SP18 & 265:08:14:00 & 47.664491 & -122.419736 & 543565 & 5279174 & 73 & 23 & 180 & 325 & 147 \\
\hline 32 & SP21 & 265:09:30:00 & 47.682955 & -122.248809 & 556378 & 5281336 & 16 & 24 & 212 & 400 & 181 \\
\hline 33 & SP22 & $265: 09: 34: 00$ & 47.651400 & -122.174900 & 561962 & 5277886 & 155 & 26 & 220 & 400 & 181 \\
\hline 34 & SP26 & $265: 09: 36: 00$ & 47.644160 & -121.946420 & 579131 & 5277289 & 158 & 21 & 260 & 500 & 226 \\
\hline 35 & SP13 & $265: 09: 38: 00$ & 47.672731 & -122.687674 & 523445 & 5279974 & 12 & 15 & 130 & 125 & 57 \\
\hline 36 & SP17 & $265: 09: 40: 00$ & 47.654140 & -122.548039 & 533939 & 5277959 & 85 & 26 & 170 & 375 & 170 \\
\hline 37 & SP20 & $265: 09: 44: 00$ & 47.650912 & -122.298710 & 552665 & 5277740 & 4 & 14 & 200 & 125 & 57 \\
\hline 38 & SP19 & $265: 11: 12: 00$ & 47.668744 & -122.345883 & 549106 & 5279691 & 79 & 18 & 190 & 25 & 11 \\
\hline
\end{tabular}


Table 4. Shot list ordered by geographic shotpoint location (west to east). Note that shotpoints are numbered from west to east, beginning with SP01 (Figure 1).

\begin{tabular}{|c|c|c|c|c|c|c|c|}
\hline $\begin{array}{l}\text { Shot } \\
\text { No. }\end{array}$ & $\begin{array}{l}\text { SP } \\
\text { No. }\end{array}$ & $\begin{array}{l}\text { Shottime UTC } \\
\text { (JD:Hr:Min:S) }\end{array}$ & Latitude & Longitude & $\begin{array}{l}\text { Ele. } \\
(\mathrm{m})\end{array}$ & $\begin{array}{r}\text { Shot size } \\
(\mathrm{lbs}) \\
\end{array}$ & Lead Shooter \\
\hline 6 & SP01 & 263:09:30:00 & 47.729520 & -123.086529 & 238 & 2800 & Reneau \\
\hline 17 & SP01 & 264:09:30:00 & 47.729520 & -123.086529 & 238 & 2800 & Reneau \\
\hline 1 & SP02 & 263:08:00:00 & 47.741223 & -123.056398 & 155 & 250 & Reneau \\
\hline 11 & SP04 & 264:08:00:00 & 47.716137 & -122.991552 & 136 & 250 & Reneau \\
\hline 7 & SP05 & 263:09:32:00 & 47.730716 & -122.947215 & 414 & 2000 & Benz \\
\hline 19 & SP05 & 264:09:34:00 & 47.729511 & -122.945819 & 404 & 2000 & Burdette \\
\hline 2 & SP06 & 263:08:02:00 & 47.707970 & -122.892340 & 5 & 50 & Benz \\
\hline 18 & SP08 & 264:09:32:00 & 47.705836 & -122.801583 & 44 & 250 & Benz \\
\hline 12 & SP09 & 264:08:02:00 & 47.693580 & -122.779130 & 86 & 50 & Benz \\
\hline 14 & SP10 & 264:08:10:00 & 47.699421 & -122.724739 & 121 & 150 & Harder \\
\hline 15 & SP11 & 264:08:12:00 & 47.680029 & -122.718209 & 117 & 500 & Kaderabek \\
\hline 22 & SP11 & 264:09:42:00 & 47.679773 & -122.718313 & 117 & 500 & Kaderabek \\
\hline 21 & SP12 & 264:09:38:00 & 47.675732 & -122.740495 & 84 & 500 & Criley \\
\hline 24 & SP12 & 264:11:08:00 & 47.675732 & -122.740495 & 84 & 500 & Criley \\
\hline 35 & SP13 & 265:09:38:00 & 47.672731 & -122.687674 & 12 & 125 & Criley \\
\hline 28 & SP14 & 265:08:08:00 & 47.677027 & -122.631557 & 73 & 50 & Criley \\
\hline 29 & SP15 & 265:08:10:00 & 47.661350 & -122.578149 & 45 & 50 & Harder \\
\hline 36 & SP17 & 265:09:40:00 & 47.654140 & -122.548039 & 85 & 375 & Harder \\
\hline 31 & SP18 & 265:08:14:00 & 47.664491 & -122.419736 & 73 & 325 & Van Schaack \\
\hline 30 & SP19 & 265:08:12:00 & 47.668744 & -122.345883 & 79 & 25 & Kaderabek \\
\hline 38 & SP19 & $265: 11: 12: 00$ & 47.668744 & -122.345883 & 79 & 25 & Kaderabek \\
\hline 37 & SP20 & 265:09:44:00 & 47.650912 & -122.298710 & 4 & 125 & Van Schaack \\
\hline 25 & SP21 & 265:08:00:00 & 47.682904 & -122.249409 & 17 & 400 & Reneau \\
\hline 32 & SP21 & 265:09:30:00 & 47.682955 & -122.248809 & 16 & 400 & Reneau \\
\hline 33 & SP22 & 265:09:34:00 & 47.651400 & -122.174900 & 155 & 400 & Burdette \\
\hline 16 & SP24 & 264:08:14:00 & 47.682420 & -122.022710 & 171 & 500 & Van Schaack \\
\hline 23 & SP24 & 264:09:44:00 & 47.682420 & -122.022710 & 171 & 500 & Van Schaack \\
\hline 27 & SP26 & 265:08:06:00 & 47.644160 & -121.946420 & 158 & 500 & Croker \\
\hline 34 & SP26 & $265: 09: 36: 00$ & 47.644160 & -121.946420 & 158 & 500 & Croker \\
\hline 26 & SP27 & 265:08:04:00 & 47.672020 & -121.930550 & 9 & 250 & Burdette \\
\hline 8 & SP29 & 263:09:34:00 & 47.657893 & -121.860752 & 129 & 800 & Burdette \\
\hline 3 & SP30 & 263:08:04:00 & 47.660151 & -121.812390 & 224 & 250 & Burdette \\
\hline 4 & SP31 & 263:08:06:00 & 47.655139 & -121.758625 & 355 & 250 & Croker \\
\hline 9 & SP32 & 263:09:36:00 & 47.651181 & -121.717902 & 389 & 2000 & Croker \\
\hline 20 & SP32 & 264:09:36:00 & 47.651181 & -121.717902 & 389 & 2000 & Croker \\
\hline 13 & SP33 & 264:08:06:00 & 47.652125 & -121.672736 & 526 & 250 & Croker \\
\hline 5 & SP34 & 263:08:08:00 & 47.653562 & -121.642726 & 467 & 250 & Criley \\
\hline 10 & SP35 & 263:11:08:00 & 47.660939 & -121.616558 & 468 & 2400 & Criley \\
\hline
\end{tabular}

Shotpoints $3,7,16,23,25$, and 28 were not used.

Shots were repeated at SP01, SP05, SP11, SP12, SP19, SP21, SP24, SP26, and SP32. 
Table 5a. Shotpoints which triggered the Pacific Northwest Seismic Network (PNSN). Event times, locations, and depths are those reported by the PNSN.

\begin{tabular}{|c|c|c|c|c|c|c|c|c|c|c|c|c|}
\hline SP No. & $\begin{array}{l}\text { DATE } \\
\text { yy/mm/dd }\end{array}$ & $\begin{array}{l}\text { Time (UT) } \\
\text { hh:mm:ss }\end{array}$ & Latitude & Longitude & $\begin{array}{l}\text { Depth } \\
(\mathrm{km})\end{array}$ & Mag & Qual. & Comment & & & & \\
\hline SP30 & $99 / 09 / 20$ & 08:04:00 & 47.66300 & 121.83933 & 0.0 & 1.2 & $\mathrm{BC}$ & 5.9 & $\mathrm{~km}$ & ENE & of & Carnation \\
\hline SP1a & $99 / 09 / 20$ & 09:30:00 & 47.70833 & 123.04433 & 1.4 & 1.5 & BB & 30.1 & $\mathrm{~km}$ & W & of & Poulsbo \\
\hline SP5a & $99 / 09 / 20$ & $09: 31: 59$ & 47.72867 & 123.02650 & 15.4 & 1.1 & $\mathrm{AD}$ & 28.6 & $\mathrm{~km}$ & W & of & Poulsbo \\
\hline SP32a & $99 / 09 / 20$ & 09:36:00 & 47.65367 & 121.70950 & 0.0 & 1.6 & BA & 15.5 & $\mathrm{~km}$ & $\mathrm{E}$ & of & Carnation \\
\hline SP35 & $99 / 09 / 20$ & 11:08:00 & 47.66417 & 121.61150 & 4.3 & 2.7 & BB & 19.2 & $\mathrm{~km}$ & WSW & of & Skykomish \\
\hline SP11a & $99 / 09 / 21$ & 08:12:01 & 47.70833 & 122.76467 & 4.1 & 1.4 & DC & 8.4 & $\mathrm{~km}$ & $\mathrm{~W}$ & of & Poulsbo \\
\hline SP5b & $99 / 09 / 21$ & 09:34:00 & 47.71833 & 122.92883 & 0.1 & 1.6 & $\mathrm{BC}$ & 21.4 & $\mathrm{~km}$ & W & of & Poulsbo \\
\hline SP32b & $99 / 09 / 21$ & $09: 36: 00$ & 47.65183 & 121.71633 & 4.0 & 1.7 & $\mathrm{CB}$ & 14.9 & $\mathrm{~km}$ & $\mathrm{E}$ & of & Carnation \\
\hline SP11b & $99 / 09 / 21$ & 09:42:01 & 47.67933 & 122.73200 & 1.3 & 1.6 & $\mathrm{AC}$ & 9.0 & $\mathrm{~km}$ & SW & of & Poulsbo \\
\hline SP21a & $99 / 09 / 22$ & 08:00:01 & 47.67400 & 122.25317 & 0.0 & 1.3 & $\mathrm{BA}$ & 3.5 & $\mathrm{~km}$ & WSW & of & Kirkland \\
\hline SP18 & $99 / 09 / 22$ & $08: 14: 02$ & 47.66467 & 122.43150 & 0.0 & 1.9 & BB & 10.7 & $\mathrm{~km}$ & NW & of & Seattle \\
\hline SP21b & $99 / 09 / 22$ & 09:30:01 & 47.68150 & 122.24617 & 0.0 & 1.7 & BA & 2.9 & $\mathrm{~km}$ & W & of & Kirkland \\
\hline SP22 & $99 / 09 / 22$ & 09:34:01 & 47.63767 & 122.17383 & 0.0 & 1.0 & $\mathrm{BC}$ & 3.6 & $\mathrm{~km}$ & NNE & of & Bellevue \\
\hline SP13 & $99 / 09 / 22$ & 09:38:01 & 47.68367 & 122.72117 & 0.7 & 1.3 & $\mathrm{AD}$ & 8.1 & $\mathrm{~km}$ & SW & of & Poulsbo \\
\hline SP17 & $99 / 09 / 22$ & 09:40:02 & 47.66067 & 122.56617 & 2.9 & 1.2 & $\mathrm{AC}$ & 10.2 & $\mathrm{~km}$ & SE & of & Poulsbo \\
\hline SP20 & $99 / 09 / 22$ & 09:44:02 & 47.63517 & 122.30333 & 0.0 & 1.9 & DB & 4.7 & $\mathrm{~km}$ & NNE & of & Seattle \\
\hline
\end{tabular}

Table 5b. Position errors of shots located by the PNSN in

Table 5a.

\begin{tabular}{lcccrr}
\hline & $\begin{array}{c}\text { Error } \\
\text { Latitude } \\
(\mathrm{km})\end{array}$ & $\begin{array}{c}\text { Error } \\
\text { Longitude } \\
(\mathrm{km})\end{array}$ & $\begin{array}{c}\text { Total } \\
\text { Range Error } \\
(\mathrm{km})\end{array}$ & $\begin{array}{c}\text { Depth } \\
\text { Error } \\
(\mathrm{km})\end{array}$ & $\begin{array}{c}\text { Magni- } \\
\text { tude }\end{array}$ \\
\hline SP1a & 2.35 & 3.31 & 4.06 & 1.4 & 1.5 \\
SP5a & 0.23 & 6.22 & 6.22 & 15.4 & 1.1 \\
SP5b & 1.24 & 1.33 & 1.82 & 0.1 & 1.6 \\
SP11a & 3.14 & 3.64 & 4.81 & 4.1 & 1.4 \\
SP11b & 0.05 & 1.07 & 1.07 & 1.3 & 1.6 \\
SP13 & 1.21 & 2.63 & 2.89 & 0.7 & 1.3 \\
SP17 & 0.72 & 1.42 & 1.60 & 2.9 & 1.2 \\
SP18 & 0.02 & 0.92 & 0.92 & 0 & 1.9 \\
SP20 & 1.75 & 0.36 & 1.78 & 0 & 1.9 \\
SP21a & 0.99 & 0.29 & 1.03 & 0 & 1.3 \\
SP21b & 0.16 & 0.21 & 0.26 & 0 & 1.7 \\
SP22 & 1.52 & 0.08 & 1.53 & 0 & 1.0 \\
SP30 & 0.32 & 2.11 & 2.14 & 0 & 1.2 \\
SP32a & 0.28 & 0.66 & 0.71 & 0 & 1.6 \\
SP32b & 0.07 & 0.12 & 0.14 & $\underline{4}$ & 1.7 \\
SP35 & $\underline{0.36}$ & $\underline{0.40}$ & $\underline{0.53}$ & $\underline{1.3}$ & $\underline{2.7}$ \\
Average & 0.90 & 1.55 & 1.97 & & 1.5 \\
\hline
\end{tabular}


TABLE 6a. Earthquakes (and Blasts) in Western Washington, September 19-22, $1999^{1}$

\begin{tabular}{|c|c|c|c|c|c|c|c|}
\hline $\begin{array}{l}\text { Event } \\
\text { Number }\end{array}$ & $\begin{array}{c}\text { Origin Time (UTC) } \\
\text { Yr:JD:Hr:Min:Sec } \\
\end{array}$ & Latitude & Longitude & $\begin{array}{c}\begin{array}{c}\text { Depth } \\
(\mathrm{km})\end{array} \\
\end{array}$ & Mag. & $\begin{array}{l}\text { Window start } \\
\text { Yr:JD:Hr:Min }\end{array}$ & $\begin{array}{l}\text { Window stop } \\
\text { Yr:JD:Hr:Min } \\
\end{array}$ \\
\hline 1 & 1999:262:04:21:44.4 & 46.440 & -119.620 & 19.9 & 3.1 & $99: 262: 04: 21$ & $99: 262: 04: 31$ \\
\hline 2 & 1999:262:05:07:56.3 & 46.449 & -119.636 & 15.8 & 0.0 & $99: 262: 05: 07$ & $99: 262: 05: 17$ \\
\hline 3 & 1999:262:06:29:41.1 & 47.575 & -121.768 & 10.2 & 1.3 & $99: 262: 06: 29$ & $99: 262: 06: 39$ \\
\hline 4 & 1999:262:11:11:52.9 & 46.390 & -120.100 & 12.4 & 3.2 & 99:262:11:11 & 99:262:11:21 \\
\hline 5 & 1999:262:11:58:21.1 & 46.460 & -120.083 & 12.6 & 1.0 & $99: 262: 11: 58$ & $99: 262: 12: 08$ \\
\hline 6 & 1999:262:17:25:35.2 & 47.964 & -121.931 & 13.0 & 0.8 & $99: 262: 17: 25$ & $99: 262: 17: 35$ \\
\hline 7 & 1999:262:18:35:08.6 & 47.268 & -123.969 & 25.3 & 0.6 & $99: 262: 18: 35$ & $99: 262: 18: 45$ \\
\hline 8 & 1999:262:19:39:57.6 & 47.815 & -119.504 & 0.0 & 1.8 & $99: 262: 19: 39$ & $99: 262: 19: 49$ \\
\hline 9 & $1999: 262: 23: 34: 26.0$ & 46.448 & -119.636 & 16.6 & 1.5 & $99: 262: 23: 34$ & $99: 262: 23: 44$ \\
\hline 10 & 1999:263:06:29:22.8 & 46.383 & -120.115 & 6.9 & 1.3 & $99: 263: 06: 29$ & $99: 263: 06: 39$ \\
\hline 11 & 1999:263:11:16:54.1 & 47.600 & -121.760 & 16.9 & 2.8 & $99: 263: 11: 16$ & $99: 263: 11: 26$ \\
\hline 12 & 1999:263:11:31:24.9 & 47.605 & -121.773 & 16.1 & 1.7 & $99: 263: 11: 31$ & $99: 263: 11: 41$ \\
\hline 13 & 1999:263:12:00:52.1 & 47.600 & -121.760 & 15.9 & 2.1 & $99: 263: 12: 00$ & $99: 263: 12: 10$ \\
\hline 14 & 1999:263:12:46:21.7 & 46.389 & -120.106 & 7.7 & 1.6 & $99: 263: 12: 46$ & $99: 263: 12: 56$ \\
\hline 15 & 1999:263:22:26:02.3 & 46.460 & -119.608 & 19.4 & -0.1 & $99: 263: 22: 26$ & $99: 263: 22: 36$ \\
\hline 16 & 1999:263:23:50:32.8 & 46.398 & -120.092 & 9.7 & 0.9 & $99: 263: 23: 50$ & 99:264:00:00 \\
\hline 17 & 1999:264:09:34:00.5 & 47.718 & -122.928 & 0.0 & 1.6 & $99: 264: 09: 34$ & $99: 264: 09: 44$ \\
\hline 18 & 1999:264:09:36:00.6 & 47.652 & -121.430 & 4.0 & 1.7 & $99: 264: 09: 36$ & $99: 264: 09: 46$ \\
\hline 19 & 1999:264:09:42:01.4 & 47.679 & -122.732 & 1.3 & 1.6 & $99: 264: 09: 42$ & $99: 264: 09: 52$ \\
\hline 20 & 1999:264:13:09:17.2 & 40.619 & -124.291 & 23.0 & 3.1 & $99: 264: 13: 09$ & $99: 264: 13: 19$ \\
\hline 21 & $1999: 264: 21: 27: 29.4$ & 48.085 & -121.928 & 0.0 & 0.9 & $99: 264: 21: 17$ & $99: 264: 21: 27$ \\
\hline 22 & 1999:265:02:06:33.6 & 47.349 & -122.315 & 13.5 & 1.3 & $99: 265: 02: 06$ & $99: 265: 02: 16$ \\
\hline 23 & 1999:265:02:32:41.3 & 47.643 & -120.222 & 0.7 & 1.7 & $99: 265: 02: 32$ & $99: 265: 02: 42$ \\
\hline 24 & 1999:265:09:56:16.7 & 47.640 & -127.193 & 10.0 & 0.0 & $99: 265: 09: 56$ & $99: 265: 10: 06$ \\
\hline 25 & 1999:265:10:01:20.5 & 48.612 & -122.170 & 3.0 & 0.7 & $99: 265: 10: 01$ & $99: 265: 10: 11$ \\
\hline 26 & 1999:265:10:21:52.2 & 45.890 & -118.190 & 5.2 & 2.3 & $99: 265: 10: 21$ & $99: 265: 10: 31$ \\
\hline
\end{tabular}

${ }^{1}$ http://www.geophys.washington.edu/SEIS/PNSN/CATALOG_SEARCH/cat.search.html

Note: Events 17-19 on the archival tapes correspond to Dry SHIPS shots SP05b, SP32b, and SP11.

The correct range to station 2144 for events 11 and 13 is $60.436 \mathrm{~km}$, and is given incorrectly in the tape header.

TABLE 6b. Teleseisms recorded September 19-22, 1999

\begin{tabular}{|c|c|c|c|c|c|c|c|c|}
\hline $\begin{array}{l}\text { Event } \\
\text { Number }\end{array}$ & $\begin{array}{c}\text { Event } \\
\text { Window }\end{array}$ & $\begin{array}{c}\text { Origin Time (UTC) } \\
\text { Yr:JD:Hr:Min:Sec }\end{array}$ & Latitude & Longitude & $\begin{array}{c}\text { Depth } \\
(\mathrm{km})\end{array}$ & Mag. & $\begin{array}{l}\text { Window start } \\
\text { Yr:JD:Hr:Min }\end{array}$ & $\begin{array}{l}\text { Window stop } \\
\text { Yr:JD:Hr:Min }\end{array}$ \\
\hline 1 & 1 & 1999:261:21:28:33.1 & 51.207 & 157.556 & 60 & 6.2 & $99: 261: 21: 30$ & $99: 261: 22: 30$ \\
\hline 2 & 2 & 1999:261:23:51:30.4 & -19.713 & 169.205 & 103 & 5.9 & 99:262:00:00 & 99:262:01:00 \\
\hline 3 & 3 & 1999:262:03:18:54.5 & -3.624 & 150.875 & 431 & 5.9 & 99:262:03:25 & $99: 262: 04: 25$ \\
\hline 4 & 4 & $1999: 263: 17: 47: 18.4$ & 23.772 & 120.982 & 33 & 7.7 & $99: 263: 17: 50$ & $99: 263: 19: 50$ \\
\hline 5 & & *1999:263:17:57:16.0 & 23.785 & 121.202 & 33 & 6.1 & & \\
\hline 6 & & *1999:263:18:03:44.2 & 23.570 & 121.299 & 33 & 6.3 & & \\
\hline 7 & & *1999:263:18:11:53.6 & 23.746 & 121.189 & 33 & 6.1 & & \\
\hline 8 & & *1999:263:18:16:18.5 & 23.756 & 121.246 & 33 & 6.2 & & \\
\hline 9 & & *1999:263:19:40:36.4 & 23.408 & 120.768 & 33 & 5.0 & & \\
\hline 10 & 5 & 1999:263:21:46:42.8 & 23.390 & 120.964 & 33 & 6.5 & $99: 263: 21: 50$ & $99: 263: 22: 50$ \\
\hline 11 & & *1999:263:21:54:49.4 & 23.584 & 120.950 & 33 & 5.3 & & \\
\hline 12 & 6 & 1999:264:11:49:46.4 & 44.715 & 149.898 & 33 & 5.7 & 99:264:11:55 & 99:264:12:55 \\
\hline 13 & 7 & 1999:264:17:38:36.8 & 23.810 & 121.320 & 14 & 5.2 & 99:264:17:51 & 99:264:18:01 \\
\hline 14 & 8 & 1999:265:00:14:39.1 & 23.729 & 121.167 & 26 & 6.4 & 99:265:00:20 & 99:265:01:20 \\
\hline 15 & & *1999:265:00:49:42.7 & 23.642 & 121.136 & 33 & 5.9 & & \\
\hline 16 & 9 & 1999:265:07:17:44.9 & 43.572 & 146.785 & 33 & 4.8 & $99: 265: 07: 25$ & 99:265:07:45 \\
\hline 17 & 10 & 1999:265:22:27:13.1 & 38.393 & -122.633 & 10 & 4.2 & $99: 265: 22: 27$ & $99: 265: 22: 37$ \\
\hline
\end{tabular}


Table 7. SEGY trace header values used for Dry SHIPS SEGY Tapes

\begin{tabular}{|c|c|c|c|}
\hline Bytes & Format & SEGY name & SHIPS header \\
\hline $\begin{array}{r}9-12 \\
13-16 \\
17-20 \\
31-32\end{array}$ & $\begin{array}{l}\text { integer } \\
\text { integer } \\
\text { integer } \\
\text { integer }\end{array}$ & $\begin{array}{l}\text { field file number (FFID) } \\
\text { trace within field record } \\
\text { source point number } \\
\text { vertical traces summed }\end{array}$ & $\begin{array}{l}\text { shot sequence number }(1-38) \\
\text { receiver station number } \\
\text { shot station number } \\
\text { instrument type: } \\
\text { 1,2,3 - Reftek vertical, N-S, E-W } \\
\text { 4 - Texan vertical } \\
5 \text { - PRS vertical } \\
6 \text { - SGR vertical } \\
7 \text { - OBS vertical } \\
8 \text { - OBS horizontal } 1 \\
9 \text { - OBS horizontal } 2 \\
10 \text { - OBS hydrophone }\end{array}$ \\
\hline $37-40$ & integer & offset & $\begin{array}{l}\text { source-receiver distance }(\mathrm{m}) \\
\text { (negative = west of shot) }\end{array}$ \\
\hline $41-44$ & integer & receiver elevation & receiver elevation $(\mathrm{m})$ \\
\hline $45-48$ & integer & source elevation & elevation at top of shot hole (m) \\
\hline $49-52$ & integer & shot depth & depth of charge below surface (m) \\
\hline $65-68$ & integer & water depth at receiver & water depth at receiver (OBS only) \\
\hline $73-76$ & integer & source $-\mathrm{x}$ & $\mathrm{x}$ coordinate at source $(\mathrm{m}, \mathrm{UTM})$ \\
\hline $77-80$ & integer & source $-\mathrm{y}$ & y coordinate at source (m, UTM) \\
\hline $81-84$ & integer & receiver $-\mathrm{x}$ & $\mathrm{x}$ coordinate at receiver (m, UTM) \\
\hline $85-88$ & integer & receiver $-\mathrm{y}$ & y coordinate at receiver (m, UTM) \\
\hline $103-104$ & int $* 2$ & total static correction & $\begin{array}{l}\text { PRS: clock drift correction (msec) } \\
\text { Reftek, Texan: } 2000 \text { msec time shift }\end{array}$ \\
\hline $105-106$ & int $* 2$ & lag time $\mathrm{A}$ to time break & PRS drift correction \\
\hline $115-116$ & int $* 2$ & samples per trace & samples per trace \\
\hline 117-118 & int $* 2$ & sample rate (microsec) & sample rate (microsec) \\
\hline $157-158$ & int $* 2$ & year & year \\
\hline $159-160$ & int $* 2$ & day & day \\
\hline $161-162$ & int $* 2$ & hour & hour at start of trace \\
\hline $163-164$ & int $* 2$ & minute & minute at start of trace \\
\hline $165-166$ & int $* 2$ & second & second at start of trace \\
\hline $167-168$ & int $* 2$ & time basis & time basis $(2=\mathrm{GMT})$ \\
\hline $173-174$ & int $* 2$ & Instrument number & See note below \\
\hline $181-184$ & Float & Shot latitude & Decimal degrees \\
\hline $185-188$ & float & Shot Longitude & Decimal degrees \\
\hline 189-192 & float & Receiver latitude & Decimal degrees \\
\hline $193-196$ & float & Receiver longitude & Decimal degrees \\
\hline
\end{tabular}




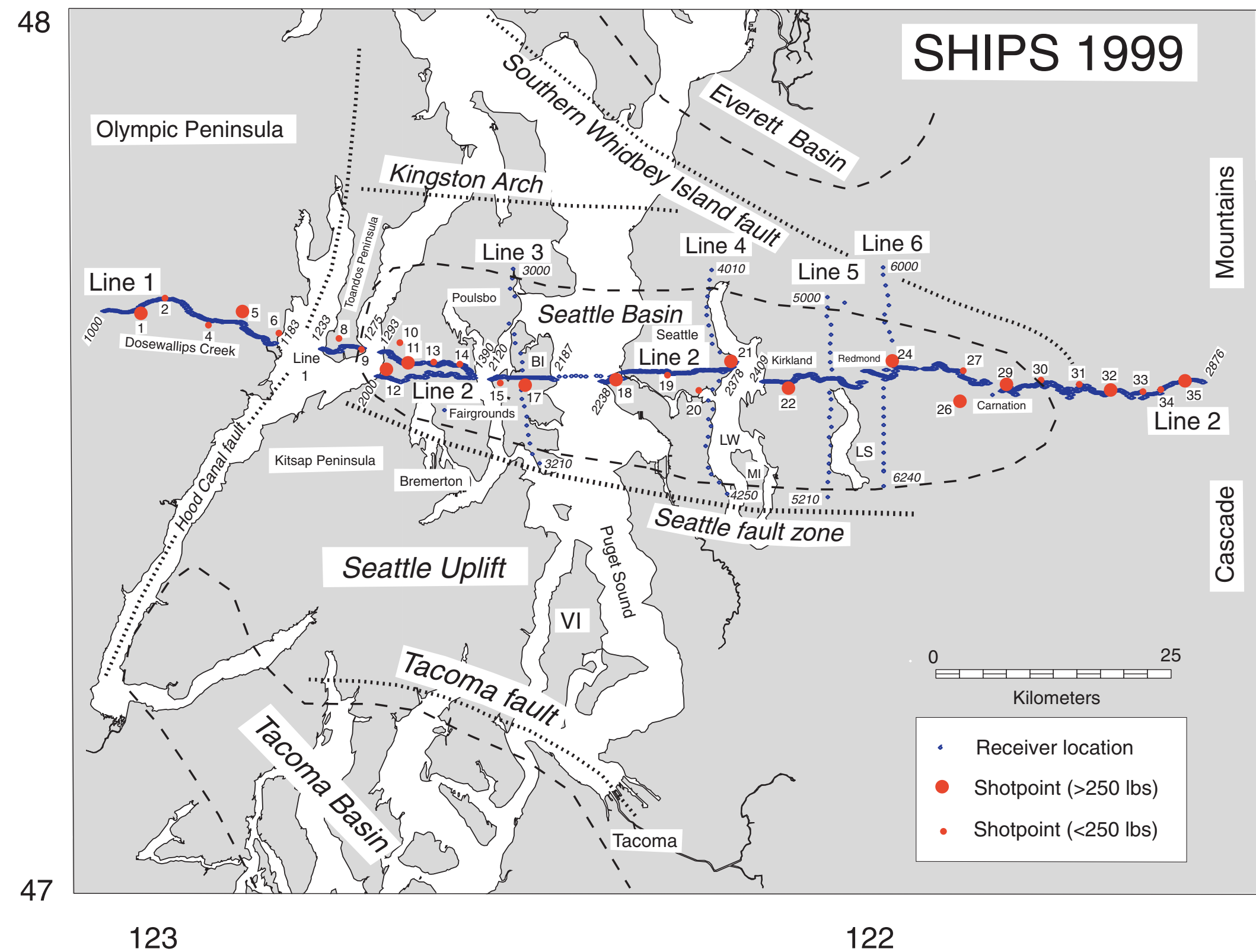

Figure 1. Map showing locations of Dry SHIP seismic shots and recorders in the Puget Lowland. Abbreviations: BI-Bainbridge Island, LS-Lake Sammamish, LW-Lake Washington, MI-Mercer Island, VI-Vashion Island. 


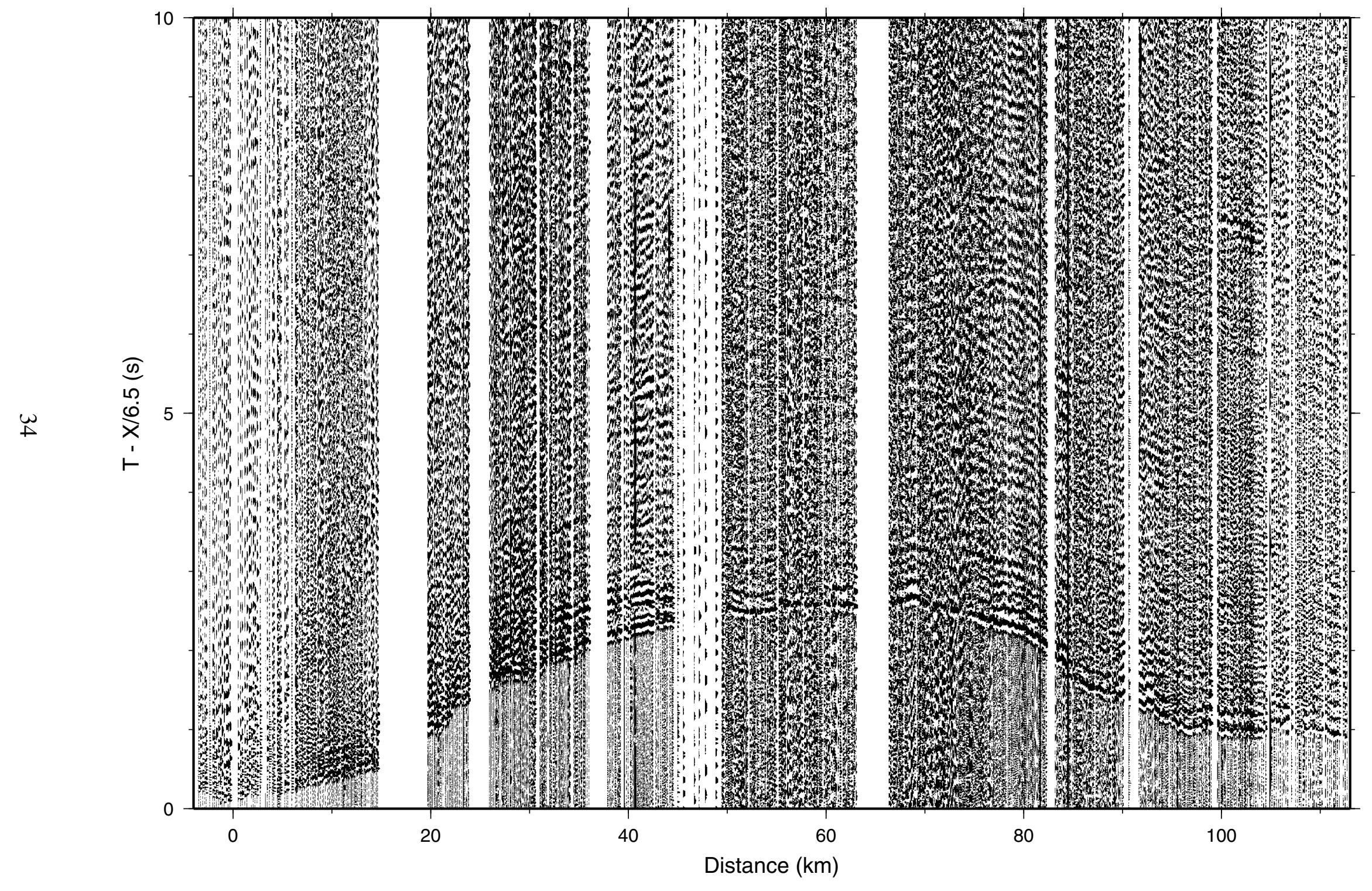

Figure 2. Reduced record section for Shotpoint 1a, vertical component only, for Lines 1 and 2. 


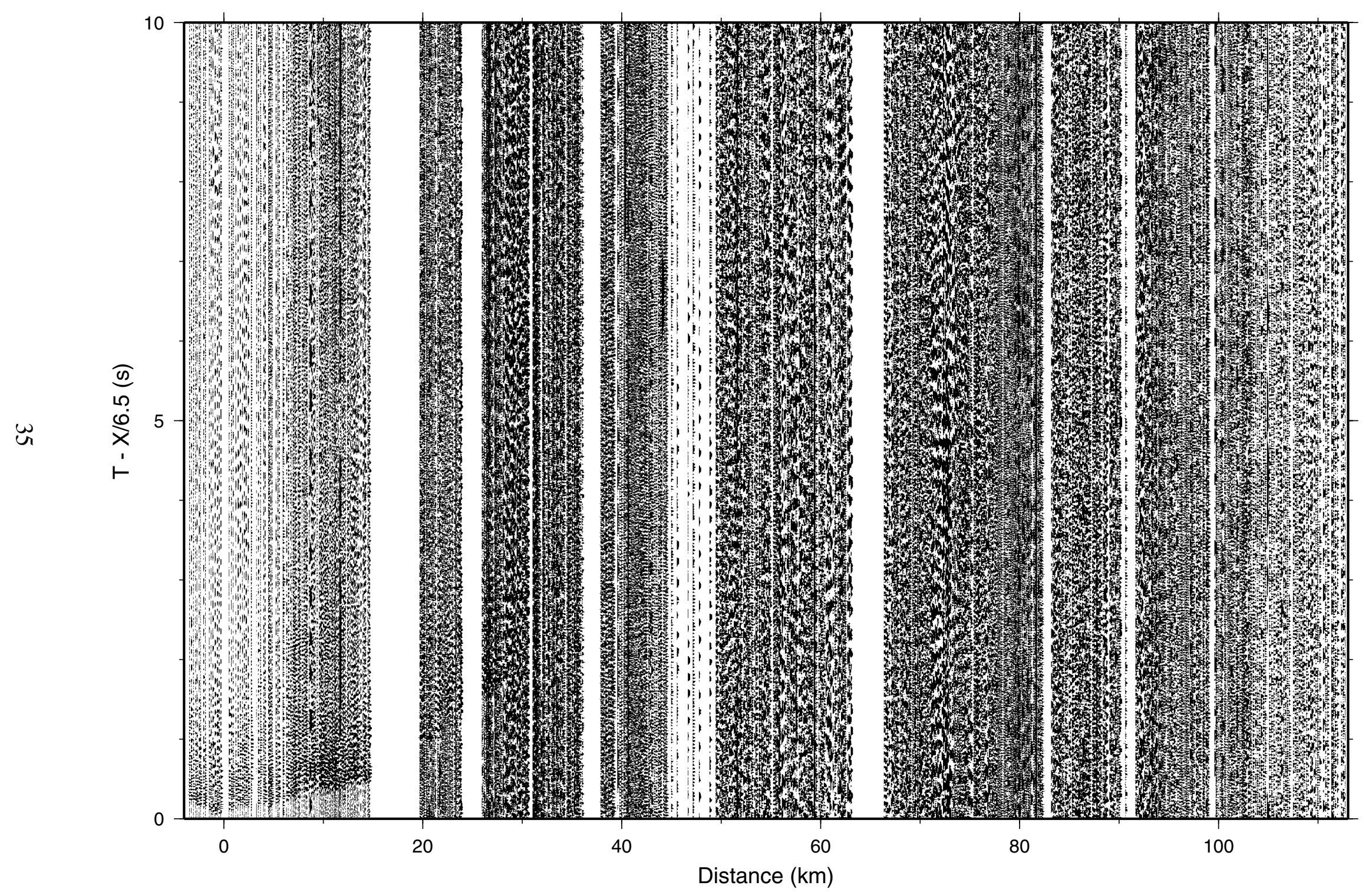

Figure 3. Reduced record section for Shotpoint 1b, vertical component only, for Lines 1 and 2. 


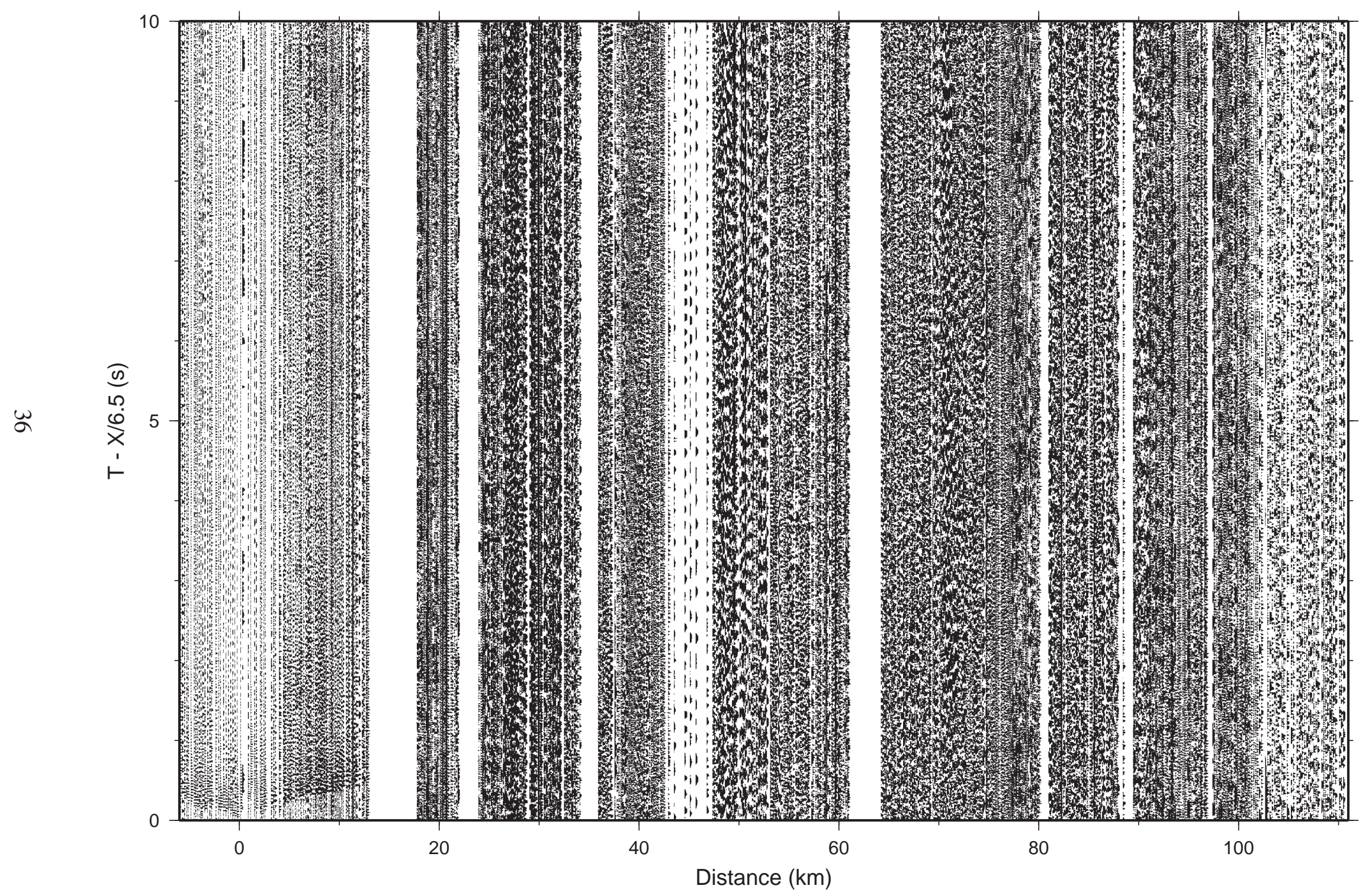

Figure 4. Reduced record section for Shotpoint 2, vertical component only, for Lines 1 and 2. 


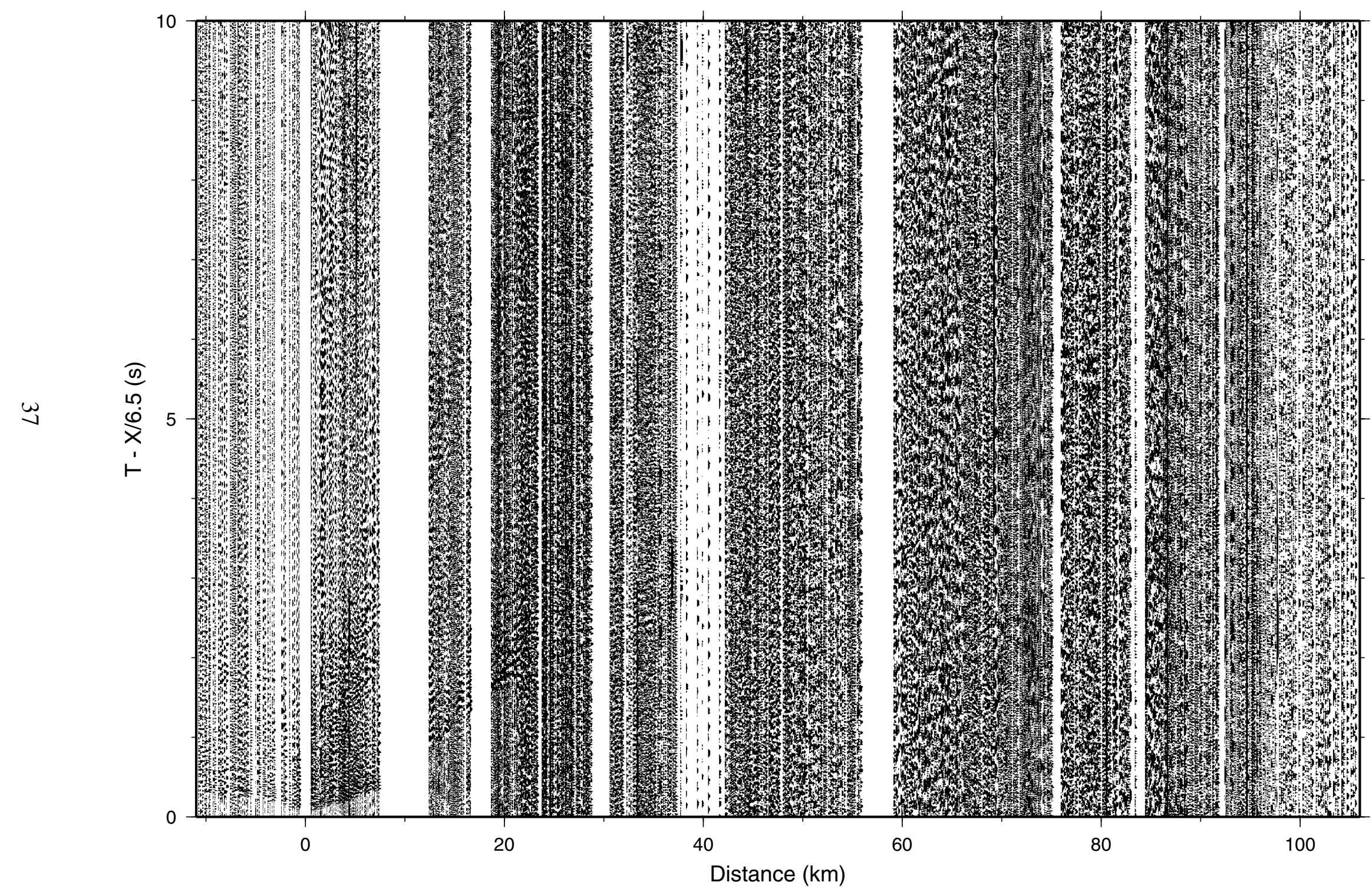

Figure 5. Reduced record section for Shotpoint 4, vertical component only, for Lines 1 and 2. 


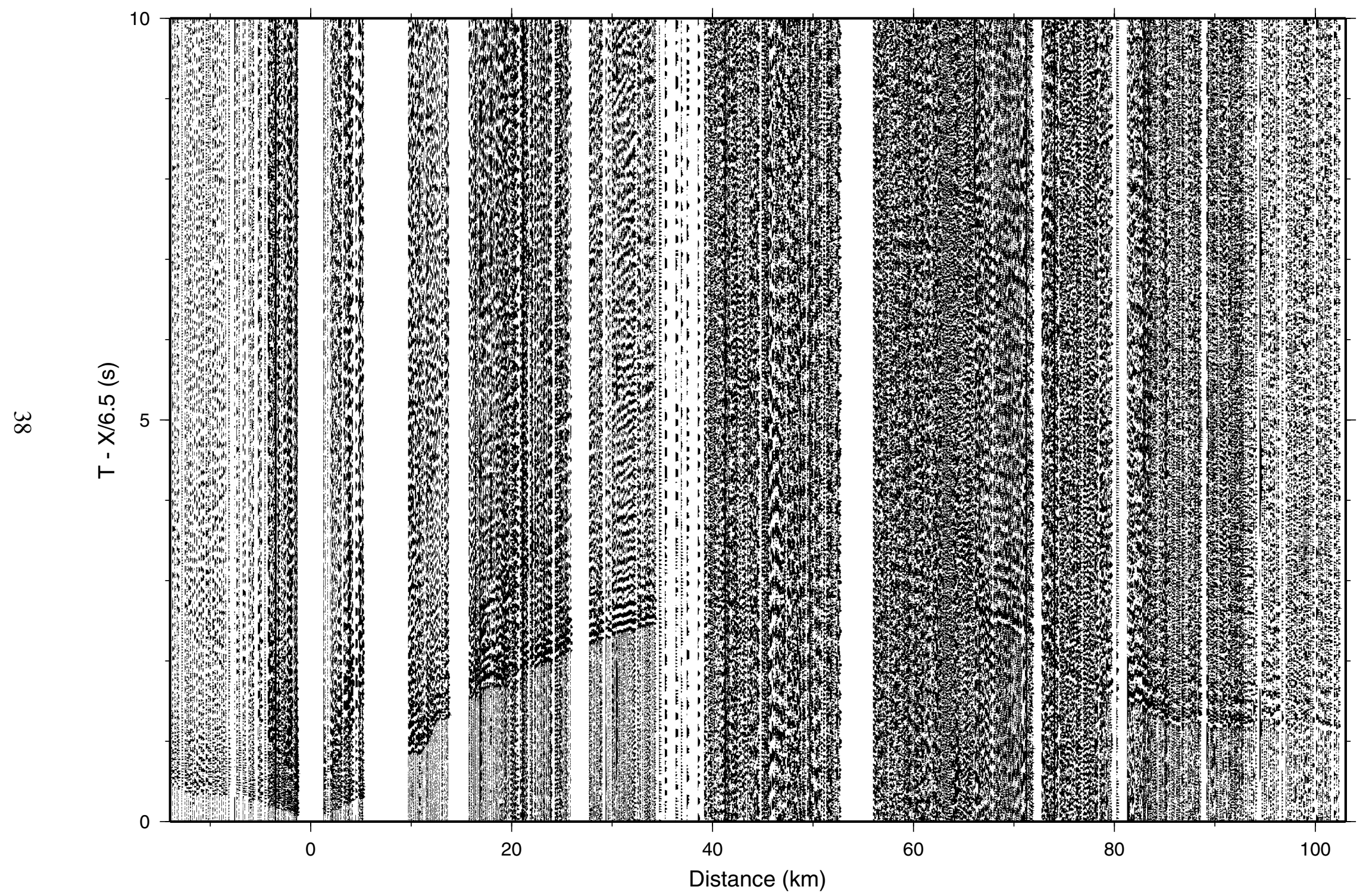

Figure 6. Reduced record section for Shotpoint 5a, vertical component only, for Lines 1 and 2. 
West

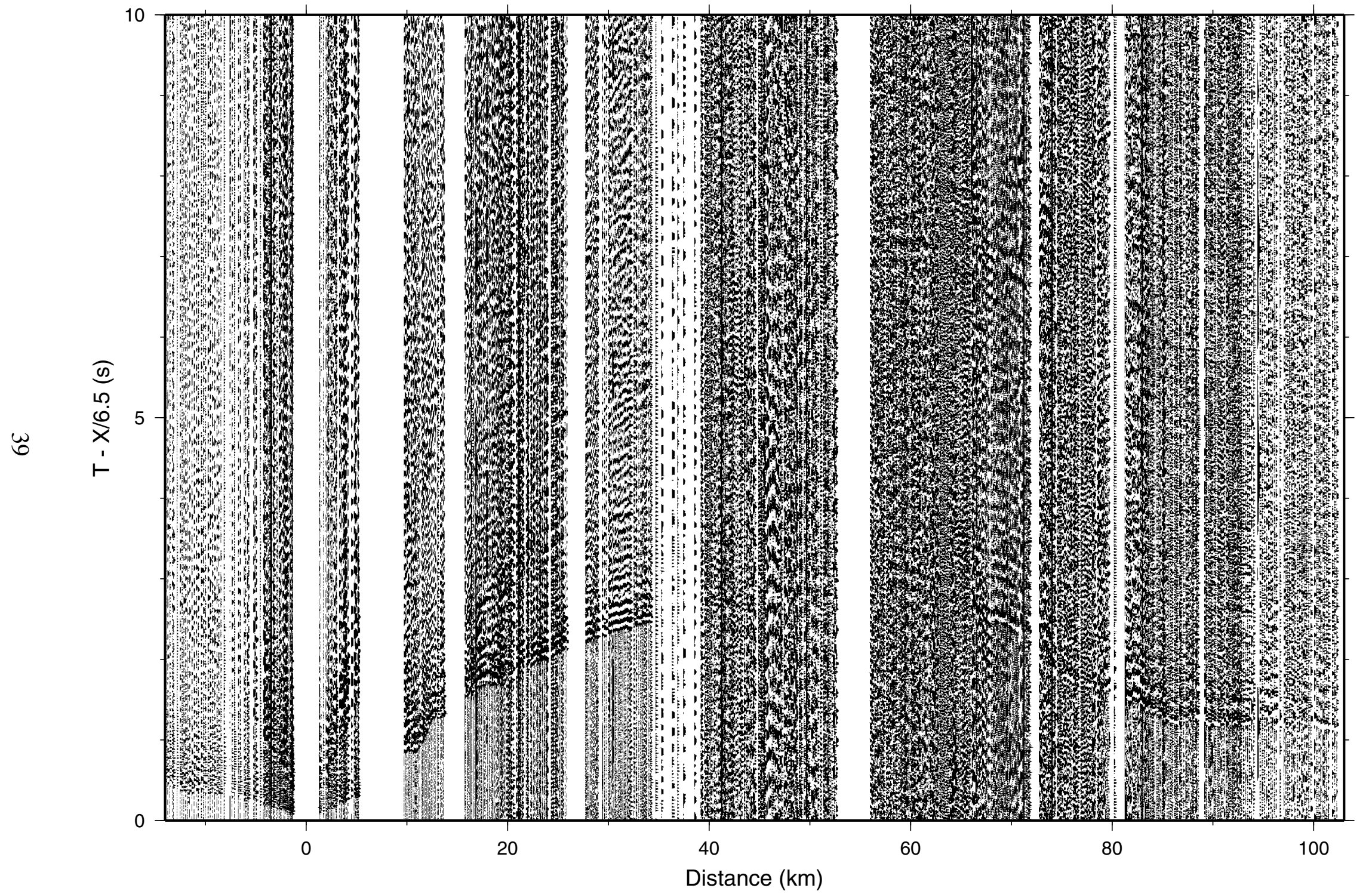

Figure 7. Reduced record section for Shotpoint 5b, vertical component only, for Lines 1 and 2. 
West

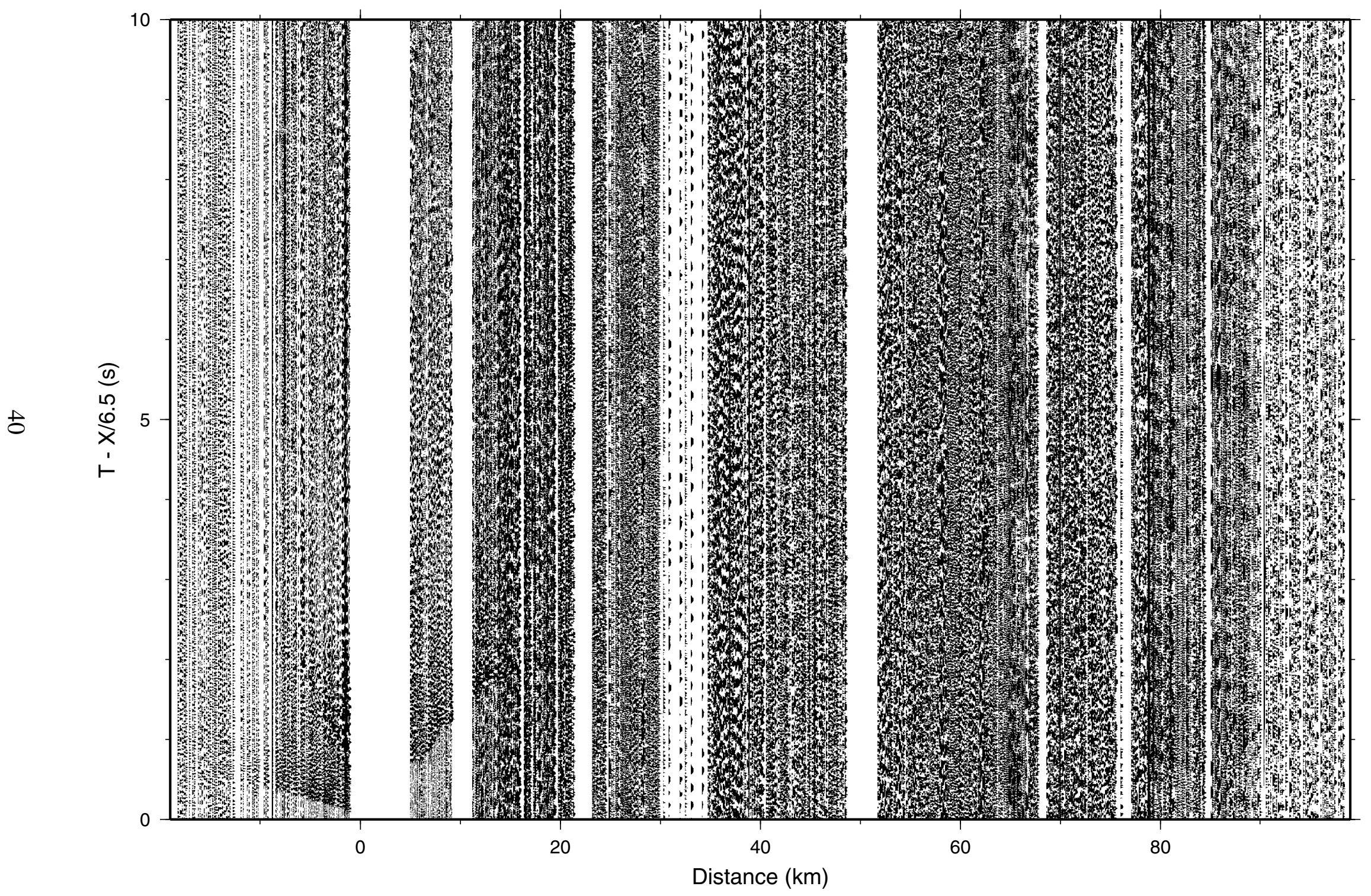

Figure 8. Reduced record section for Shotpoint 6, vertical component only, for Lines 1 and 2. 


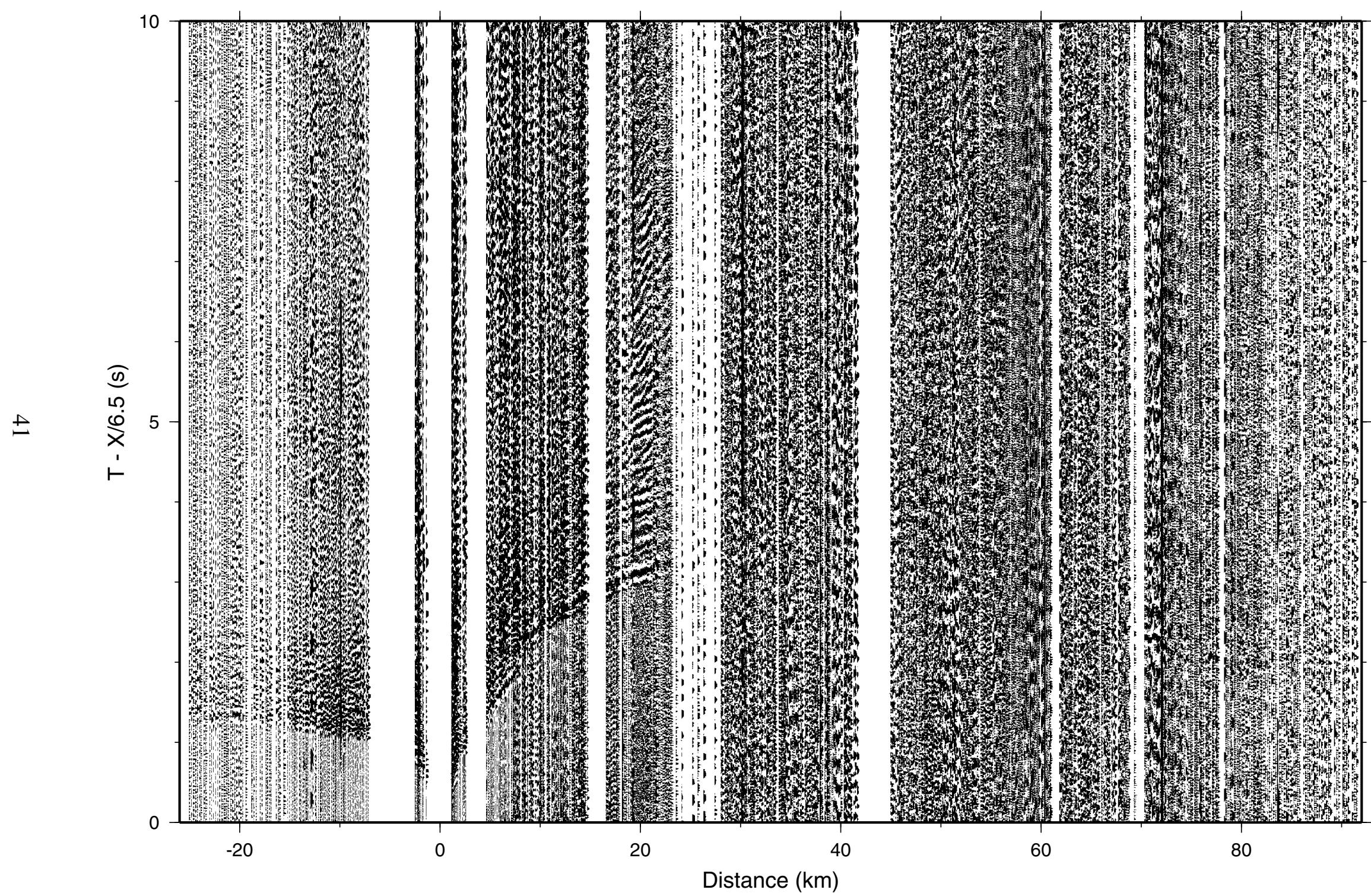

Figure 9. Reduced record section for Shotpoint 8, vertical component only, for Lines 1 and 2. 


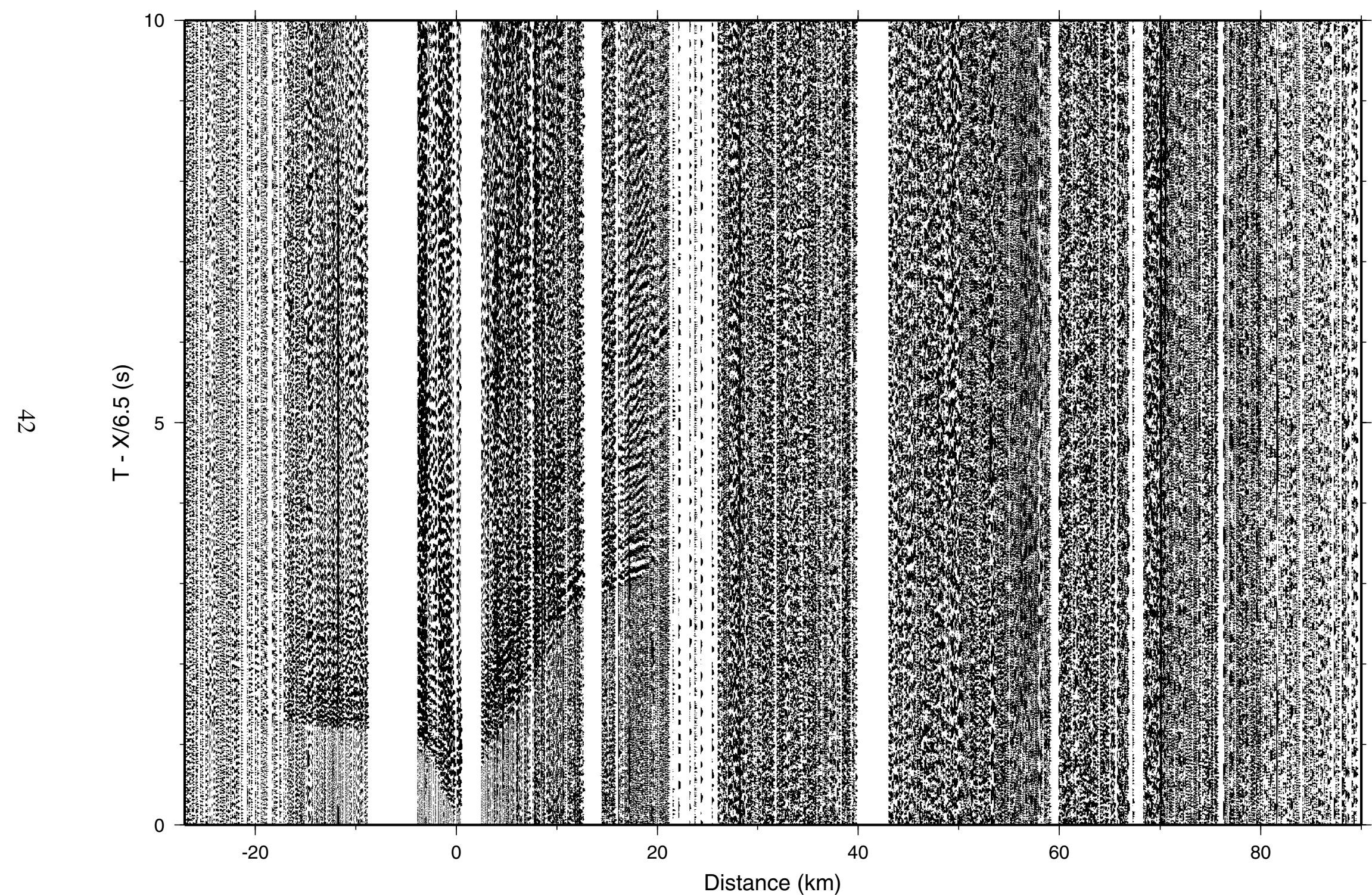

Figure 10. Reduced record section for Shotpoint 9, vertical component only, for Lines 1 and 2. 


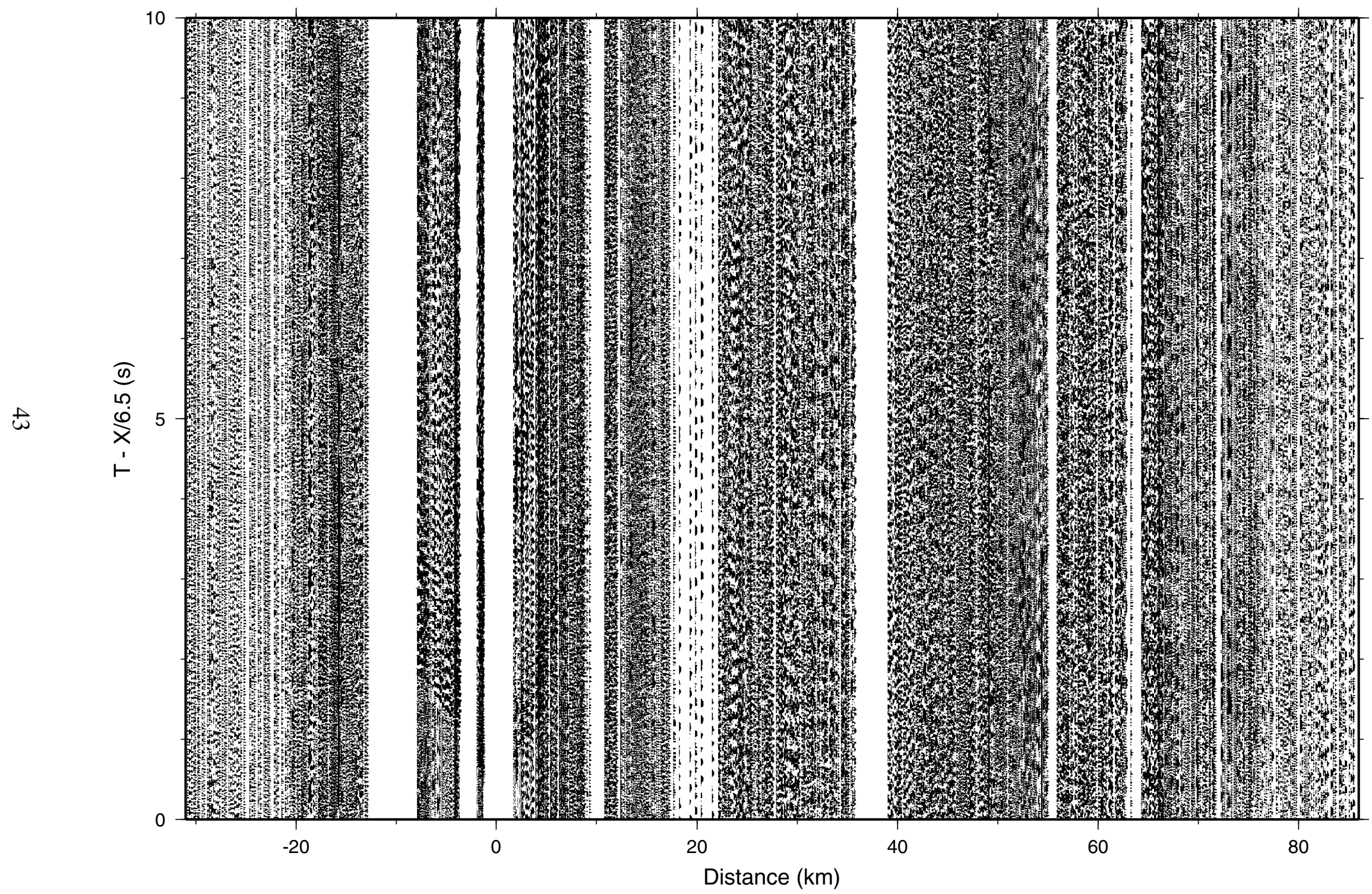

Figure 11. Reduced record section for Shotpoint 10, vertical component only, for Lines 1 and 2. 


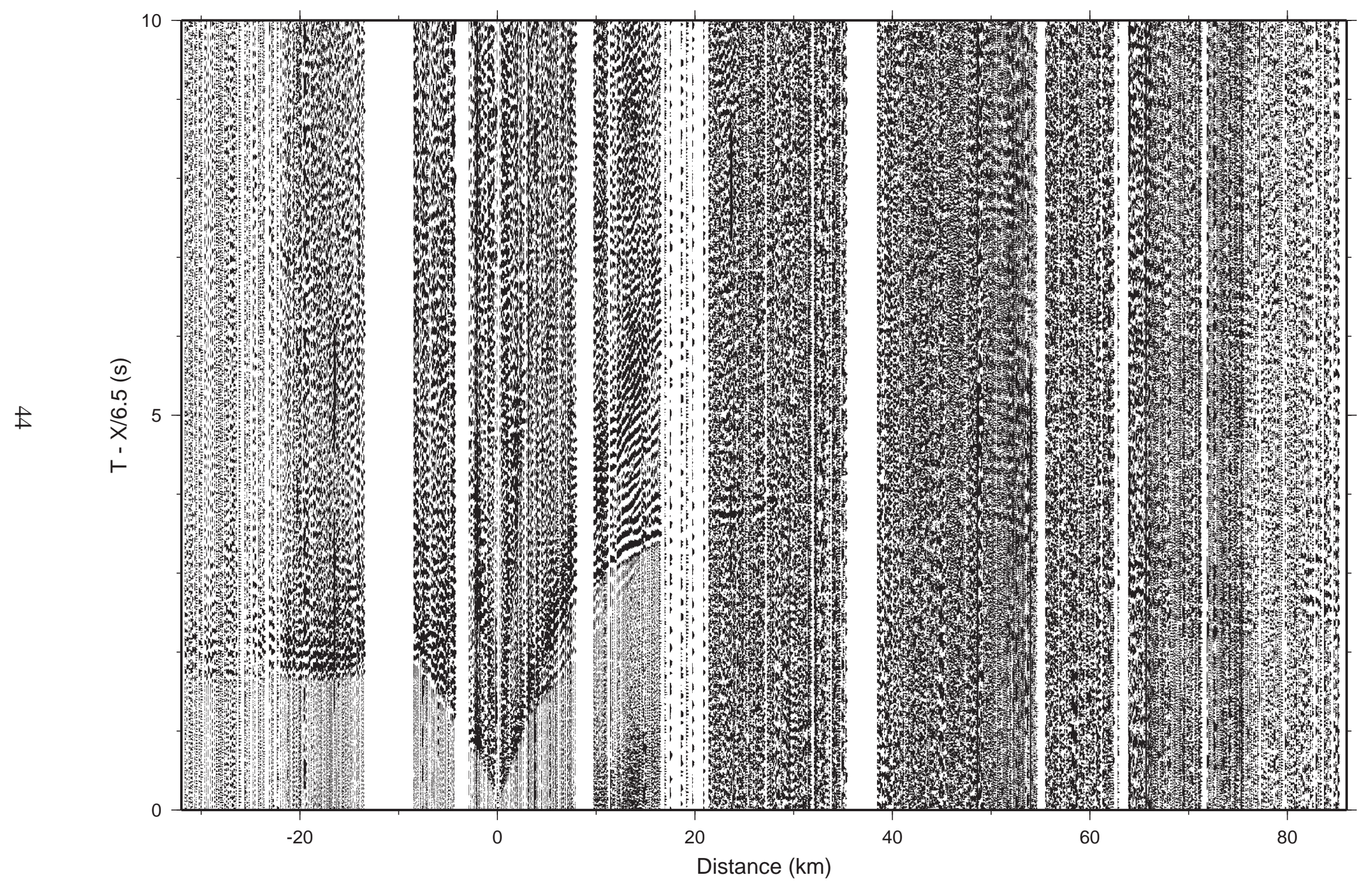

Figure 12. Reduced record section for Shotpoint 11a, vertical component only, for Lines 1 and 2. 


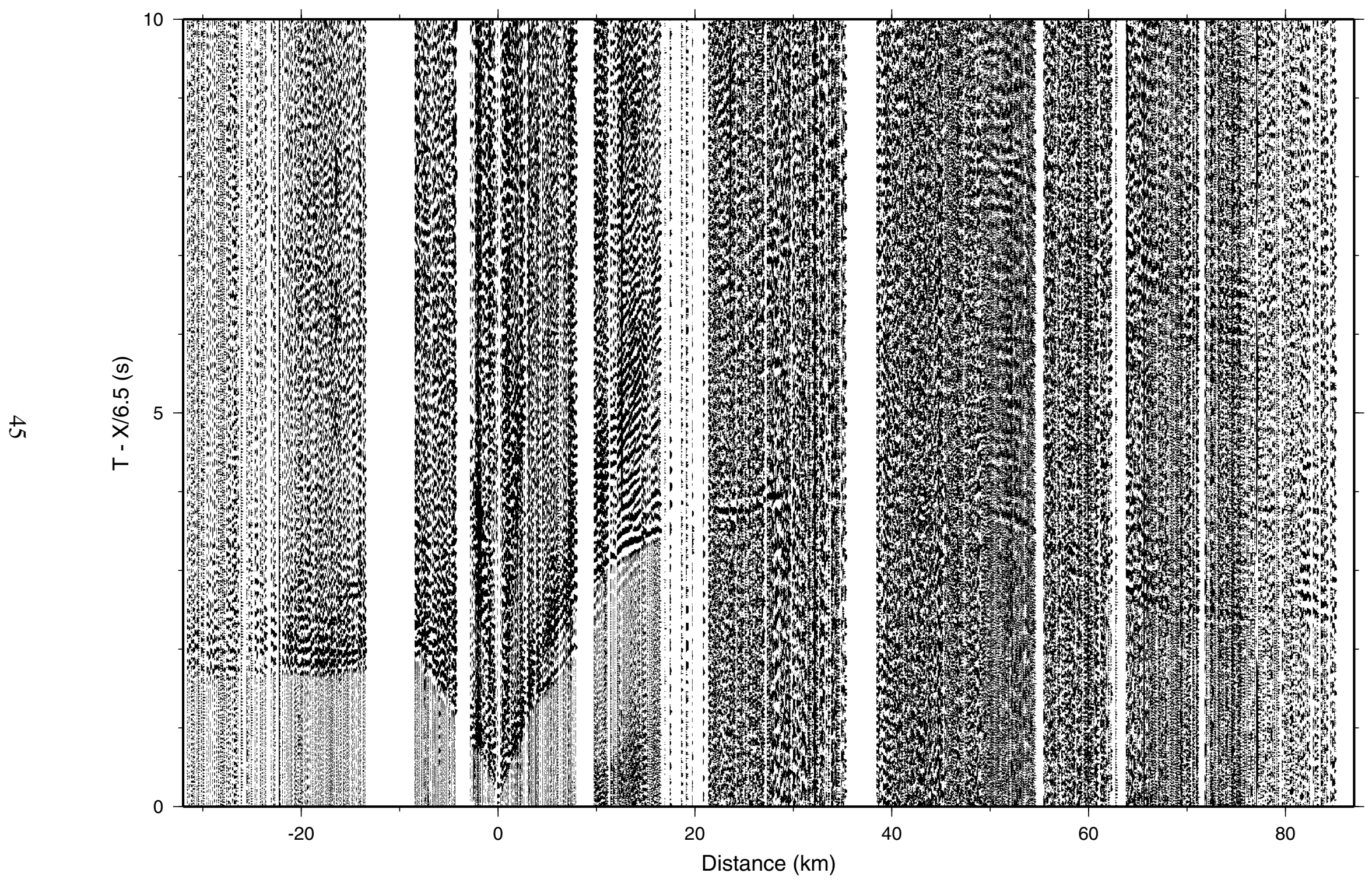

Figure 13. Reduced record section for Shotpoint 11b, vertical component only, for Lines 1 and 2. 


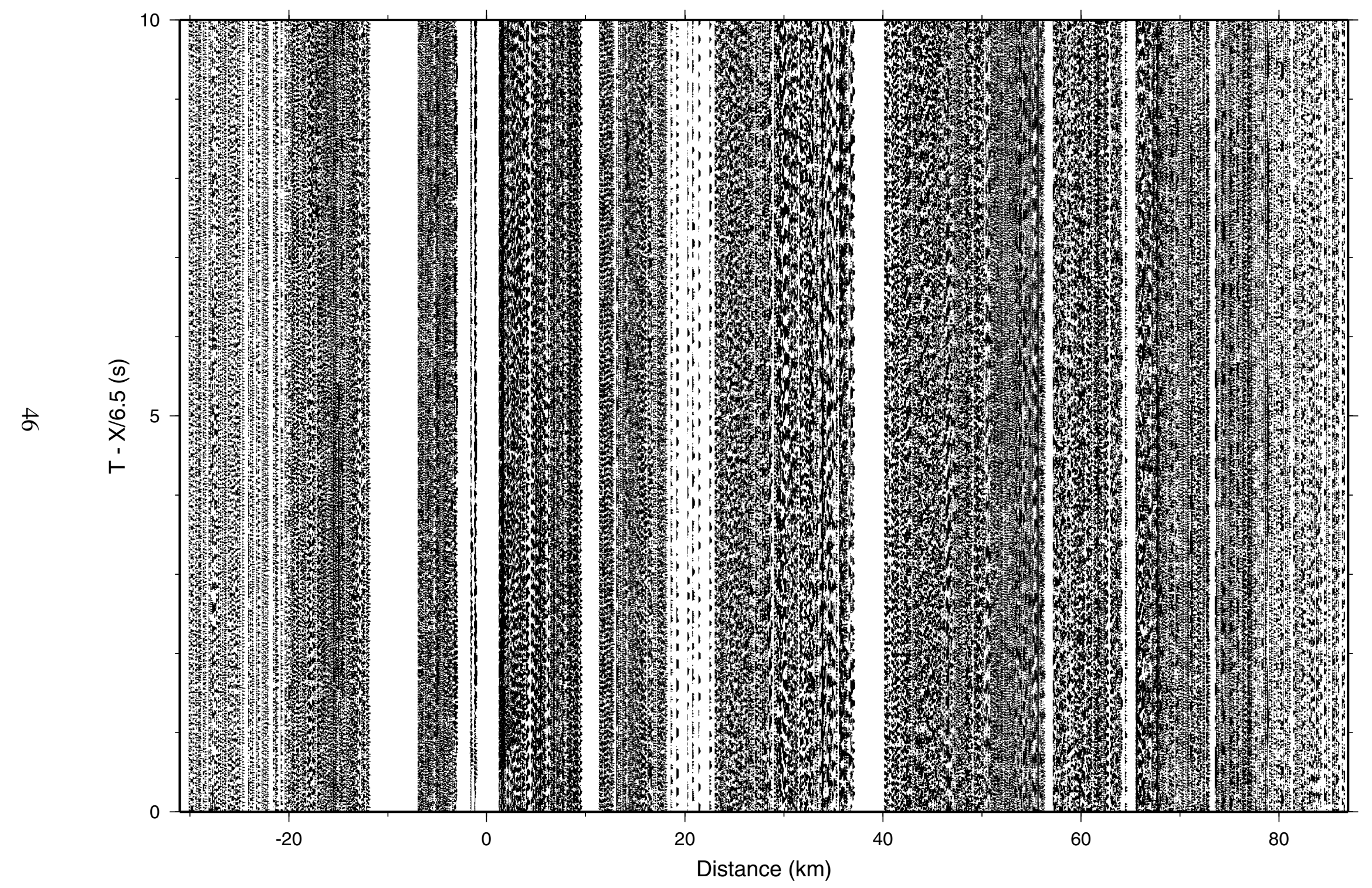

Figure 14. Reduced record section for Shotpoint 12a, vertical component only, for Lines 1 and 2. 


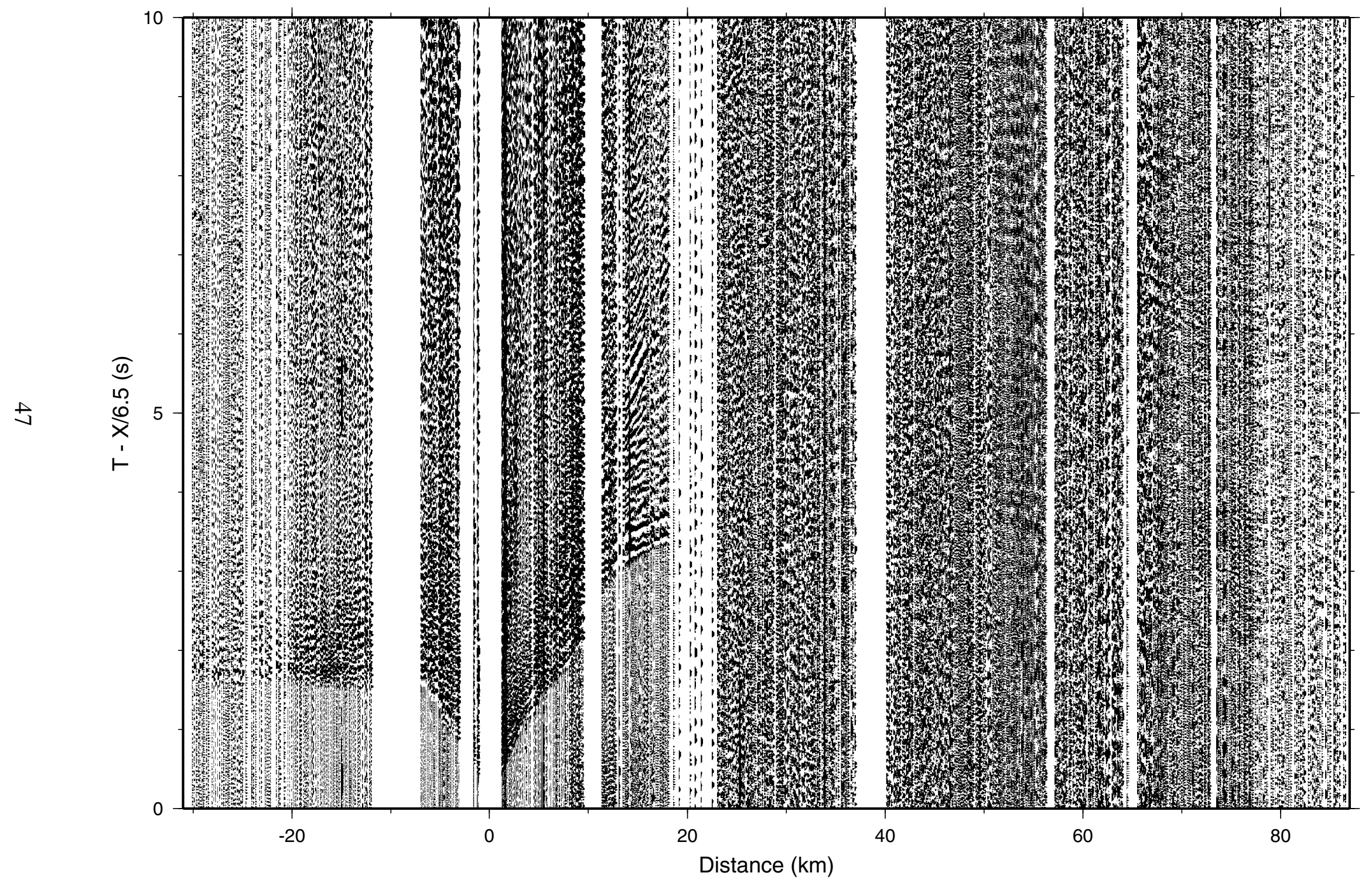

Figure 15. Reduced record section for Shotpoint 12b, vertical component only, for Lines 1 and 2. 


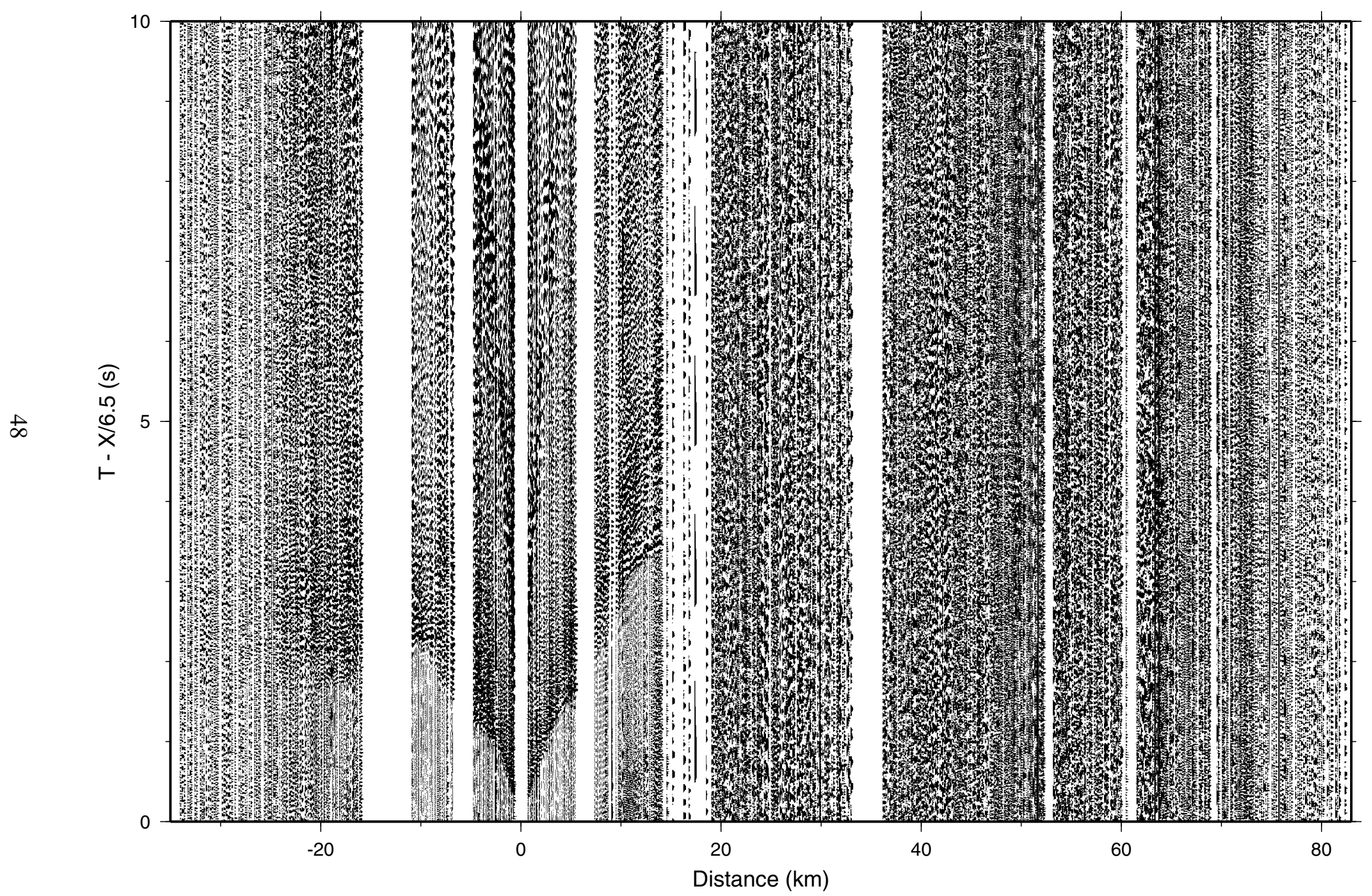

Figure 16. Reduced record section for Shotpoint 13, vertical component only, for Lines 1 and 2. 


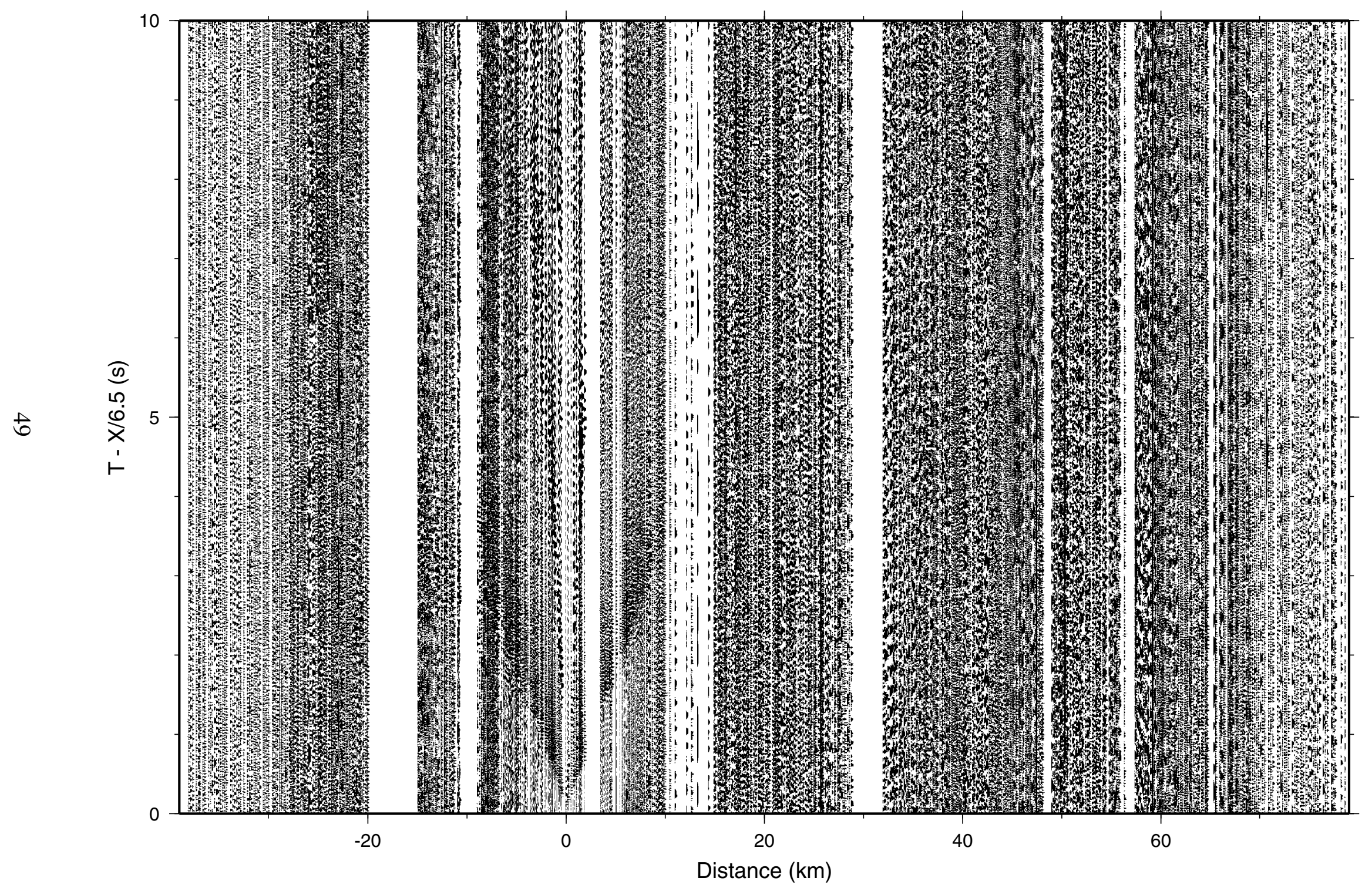

Figure 17. Reduced record section for Shotpoint 14, vertical component only, for Lines 1 and 2. 


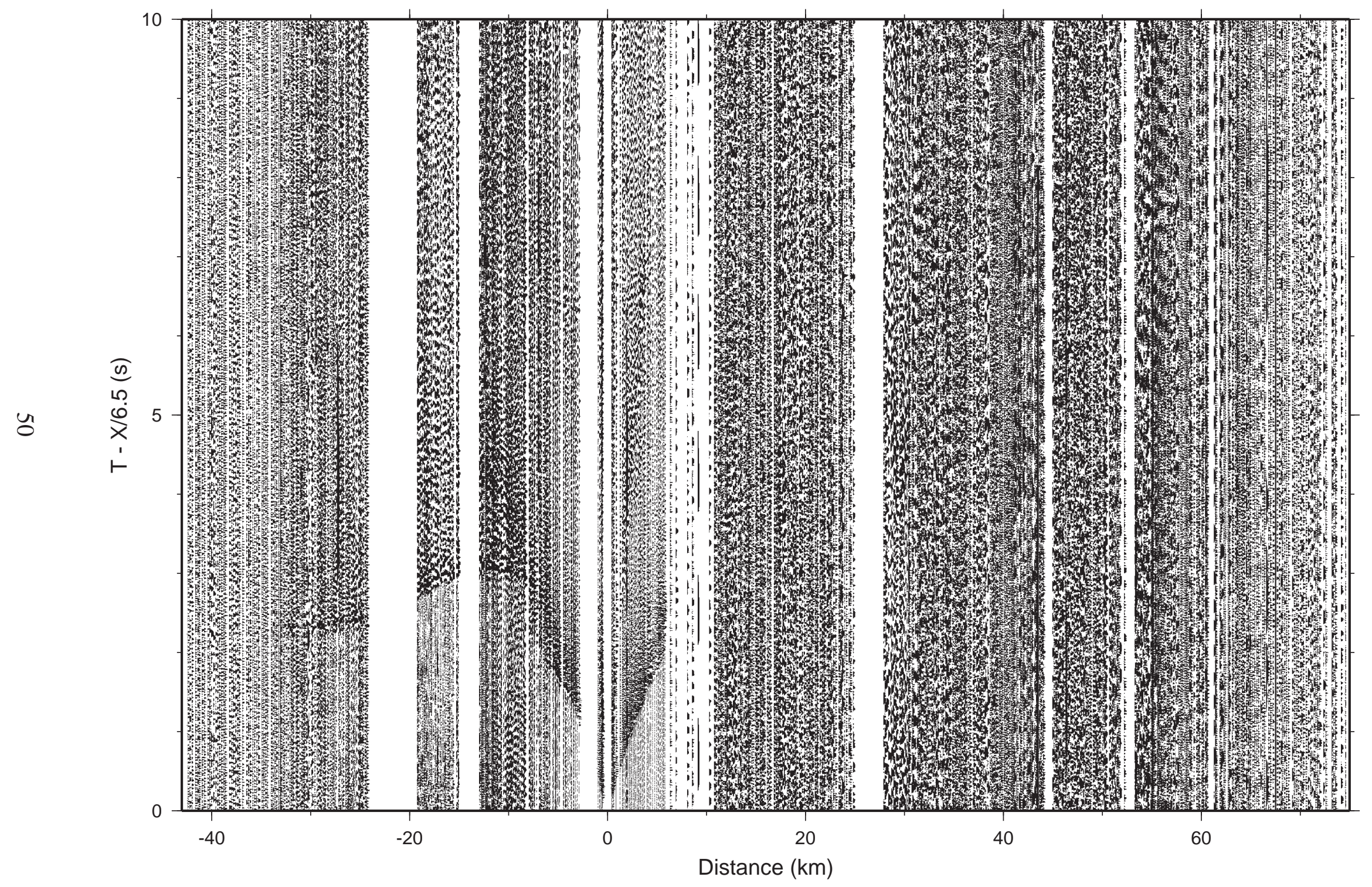

Figure 18. Reduced record section for Shotpoint 15, vertical component only, for Lines 1 and 2. 


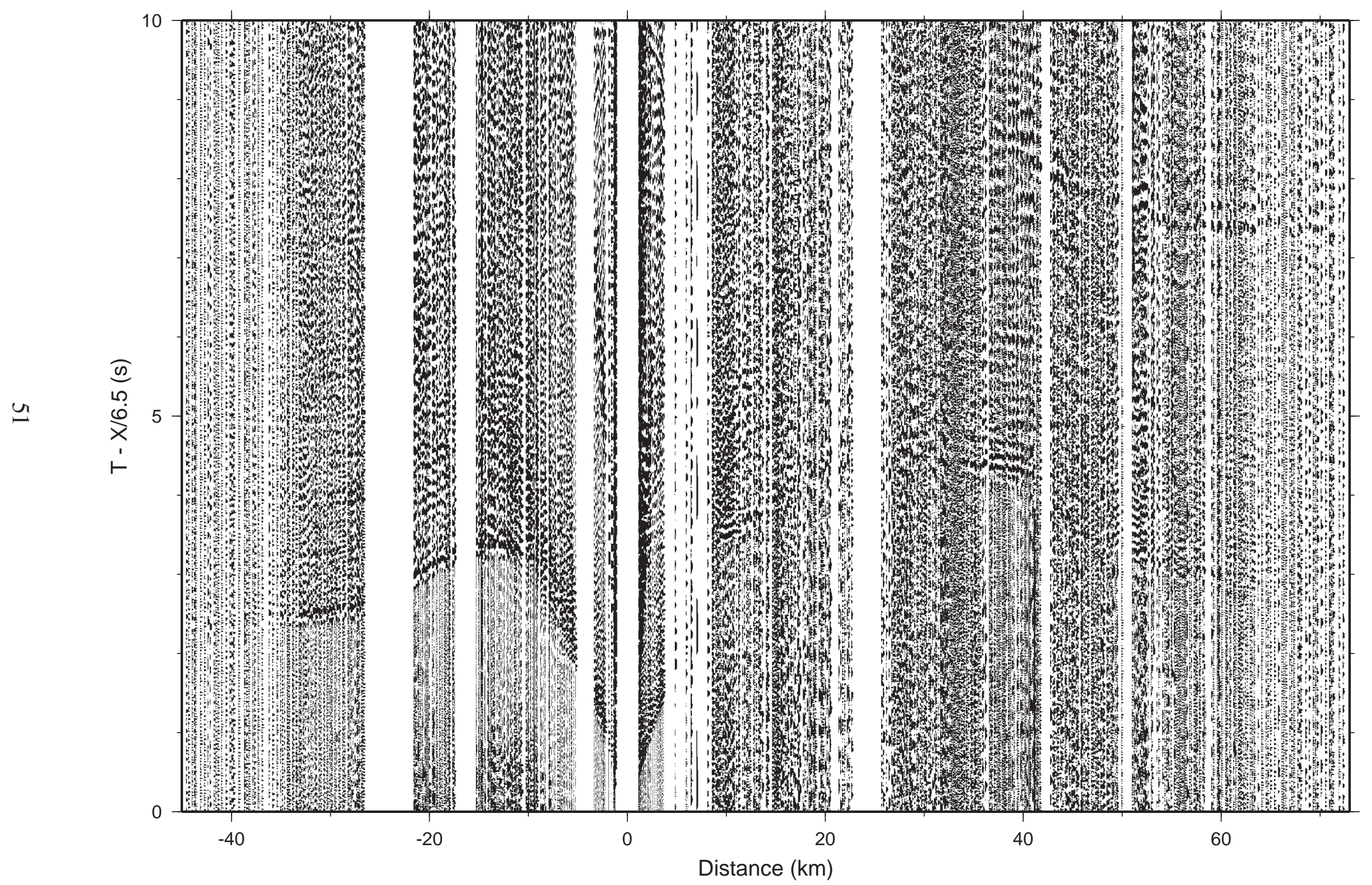

Figure 19. Reduced record section for Shotpoint 17, vertical component only, for Lines 1 and 2. 


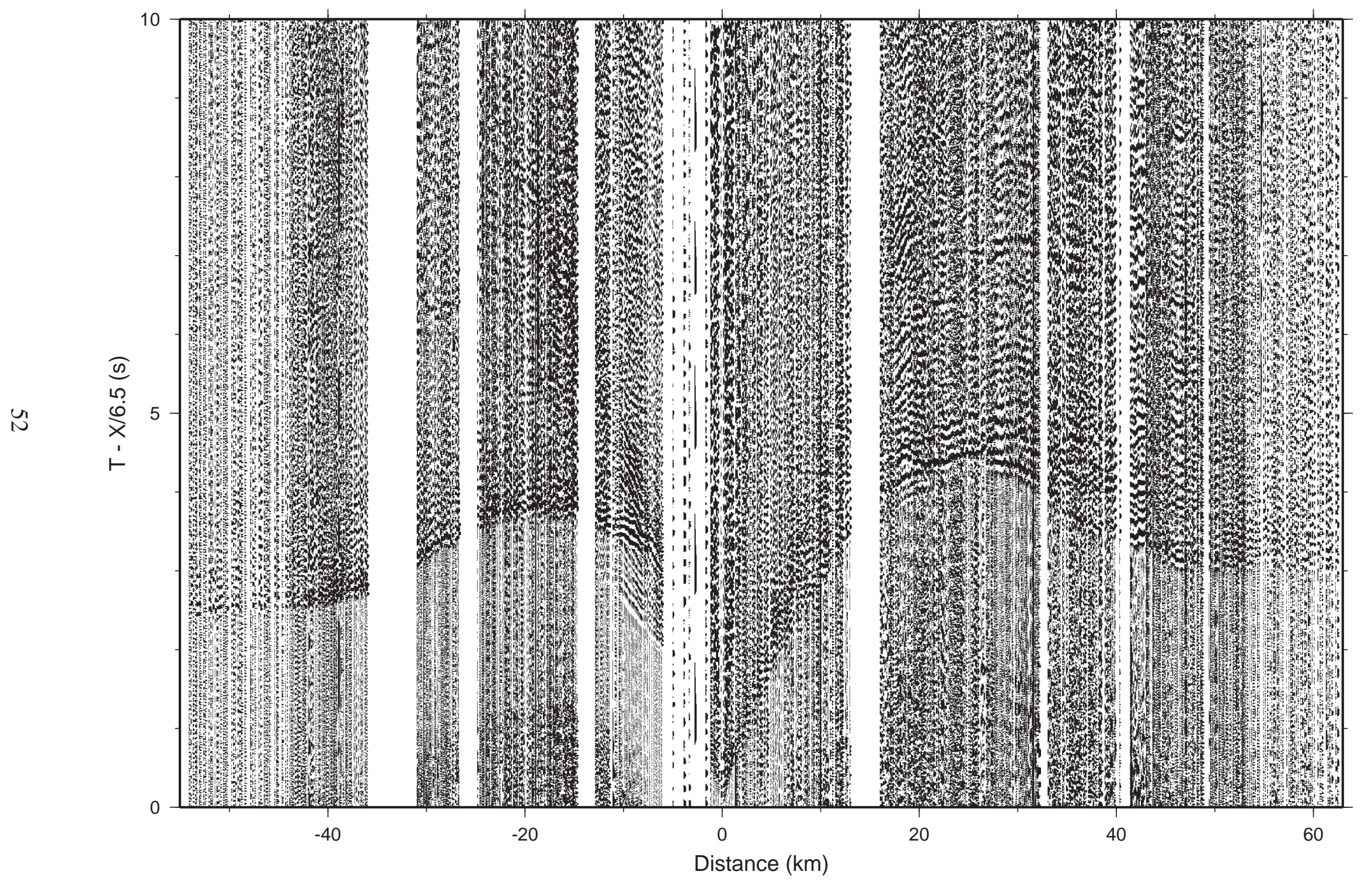

Figure 20. Reduced record section for Shotpoint 18, vertical component only, for Lines 1 and 2. 


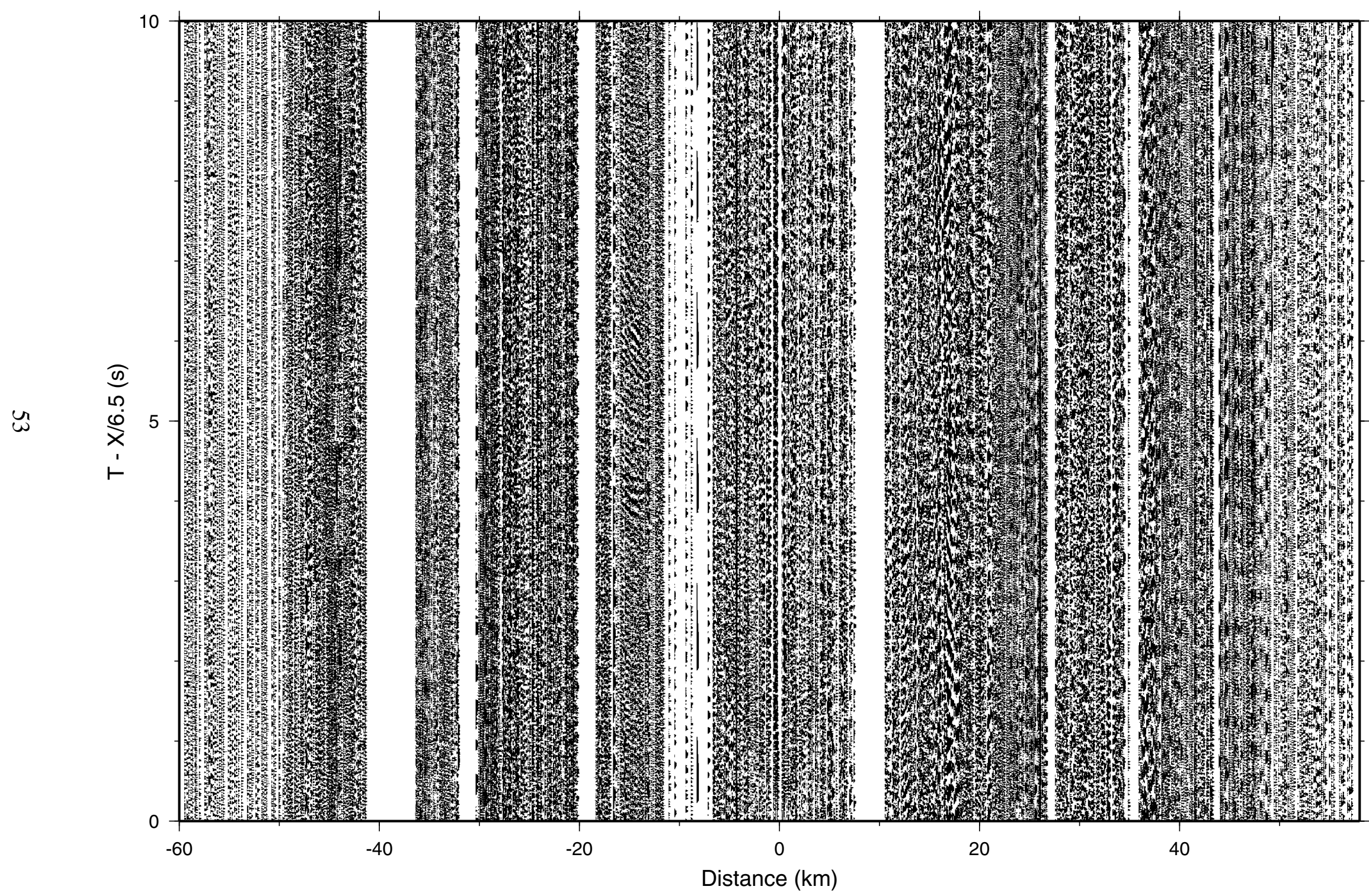

Figure 21. Reduced record section for Shotpoint 19a, vertical component only, for Lines 1 and 2. 


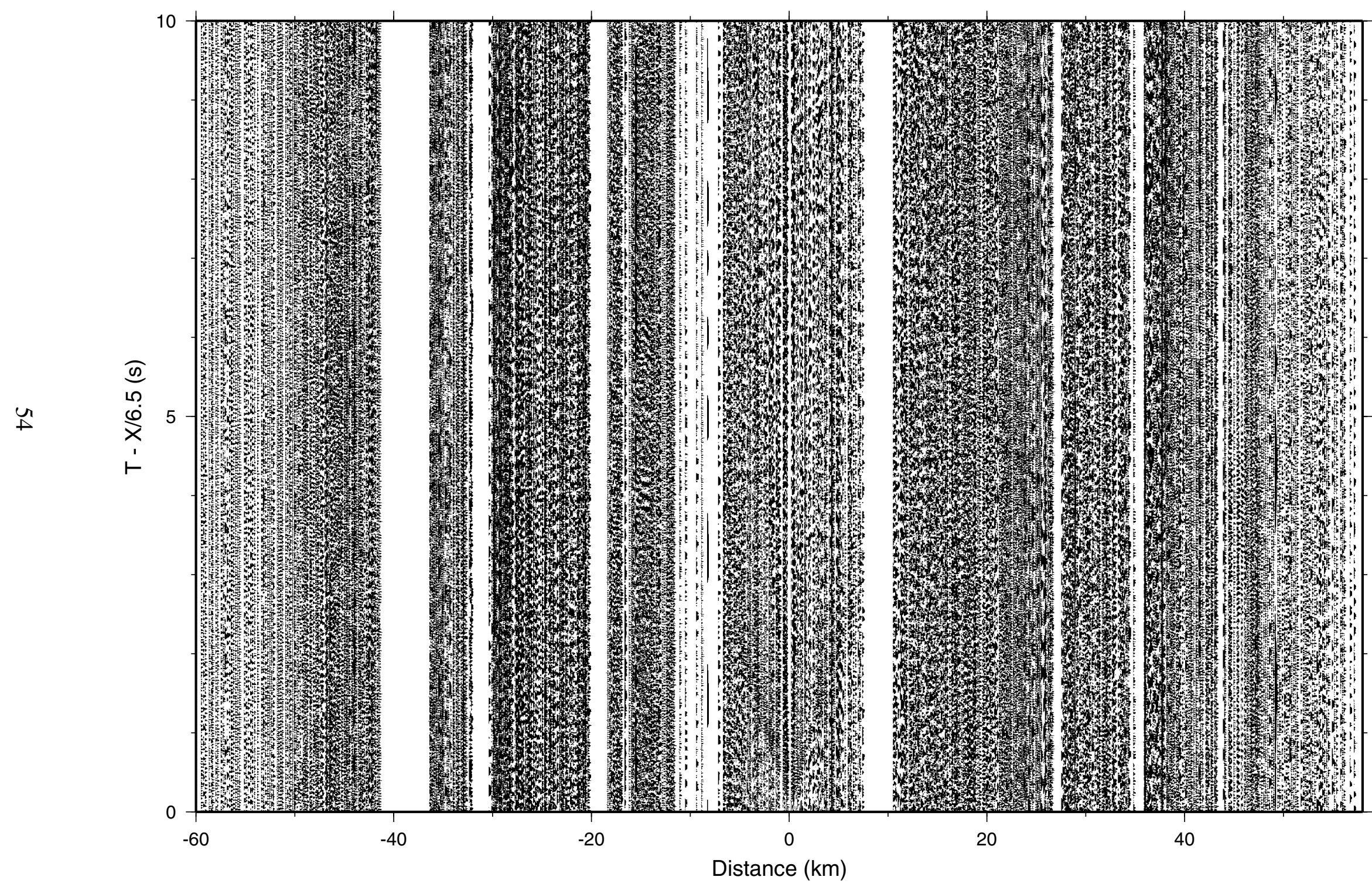

Figure 22. Reduced record section for Shotpoint 19b, vertical component only, for Lines 1 and 2. 


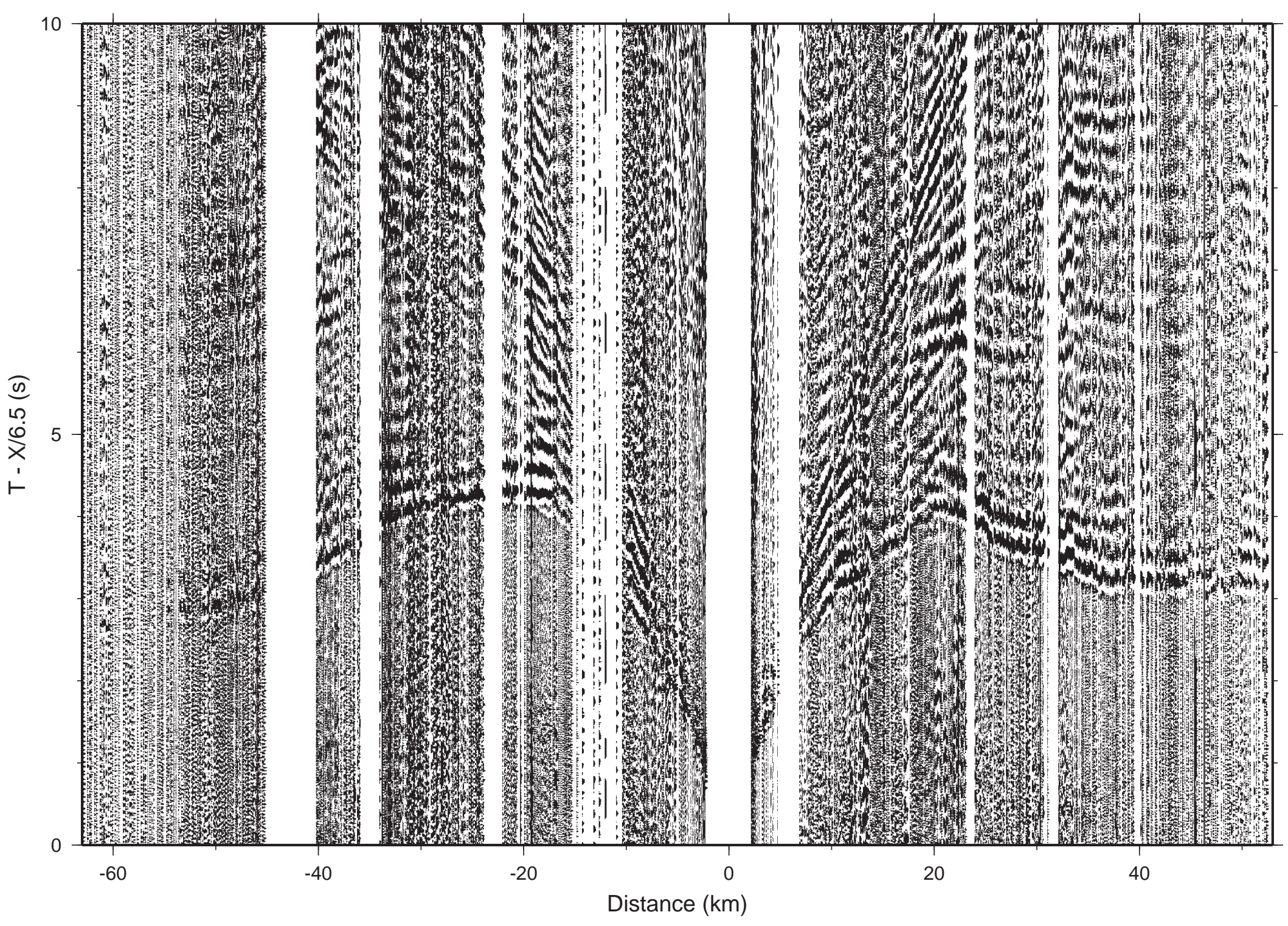

Figure 23. Reduced record section for Shotpoint 20, vertical component only, for Lines 1 and 2. 


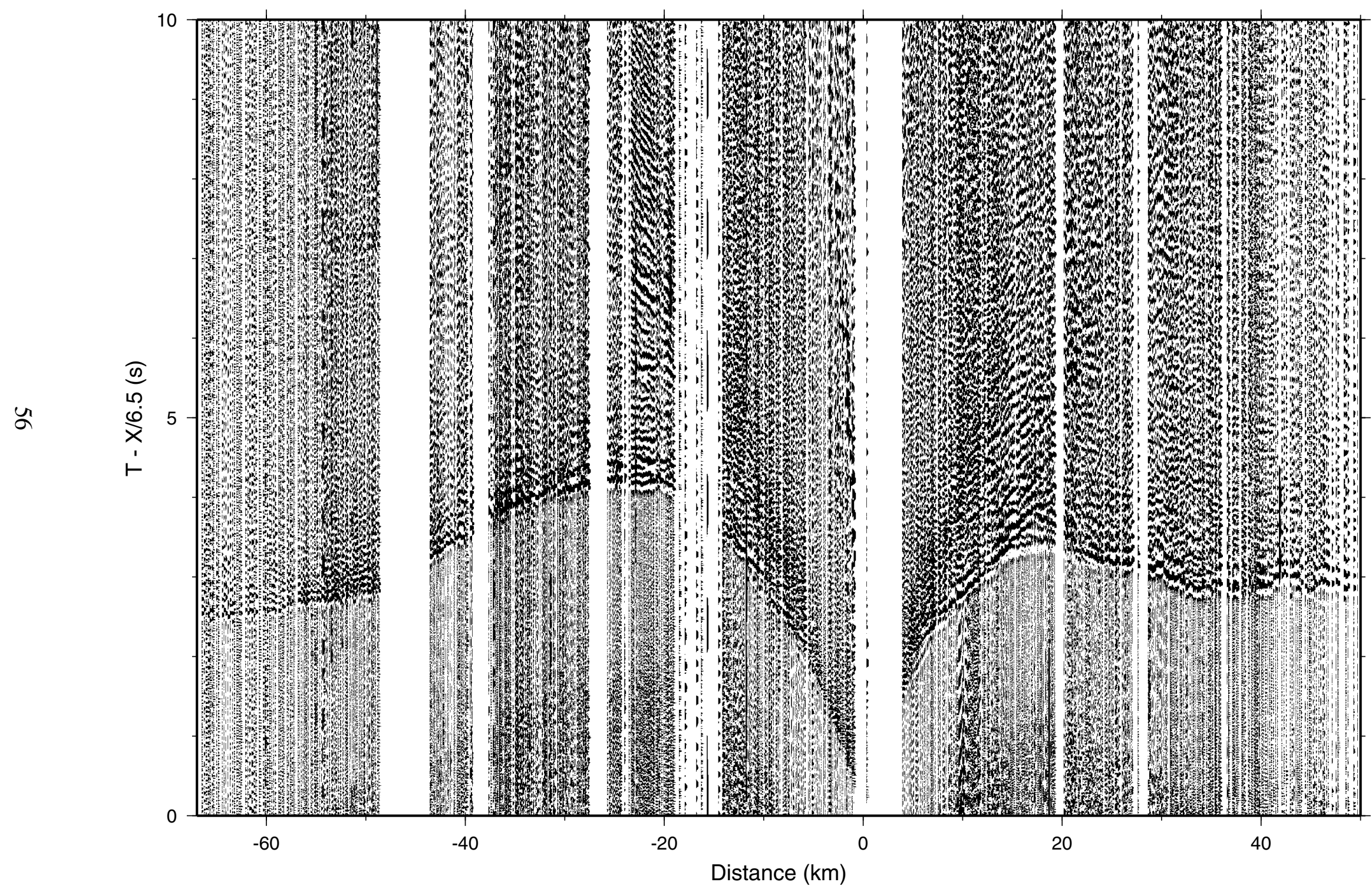

Figure 24. Reduced record section for Shotpoint 21a, vertical component only, for Lines 1 and 2. 


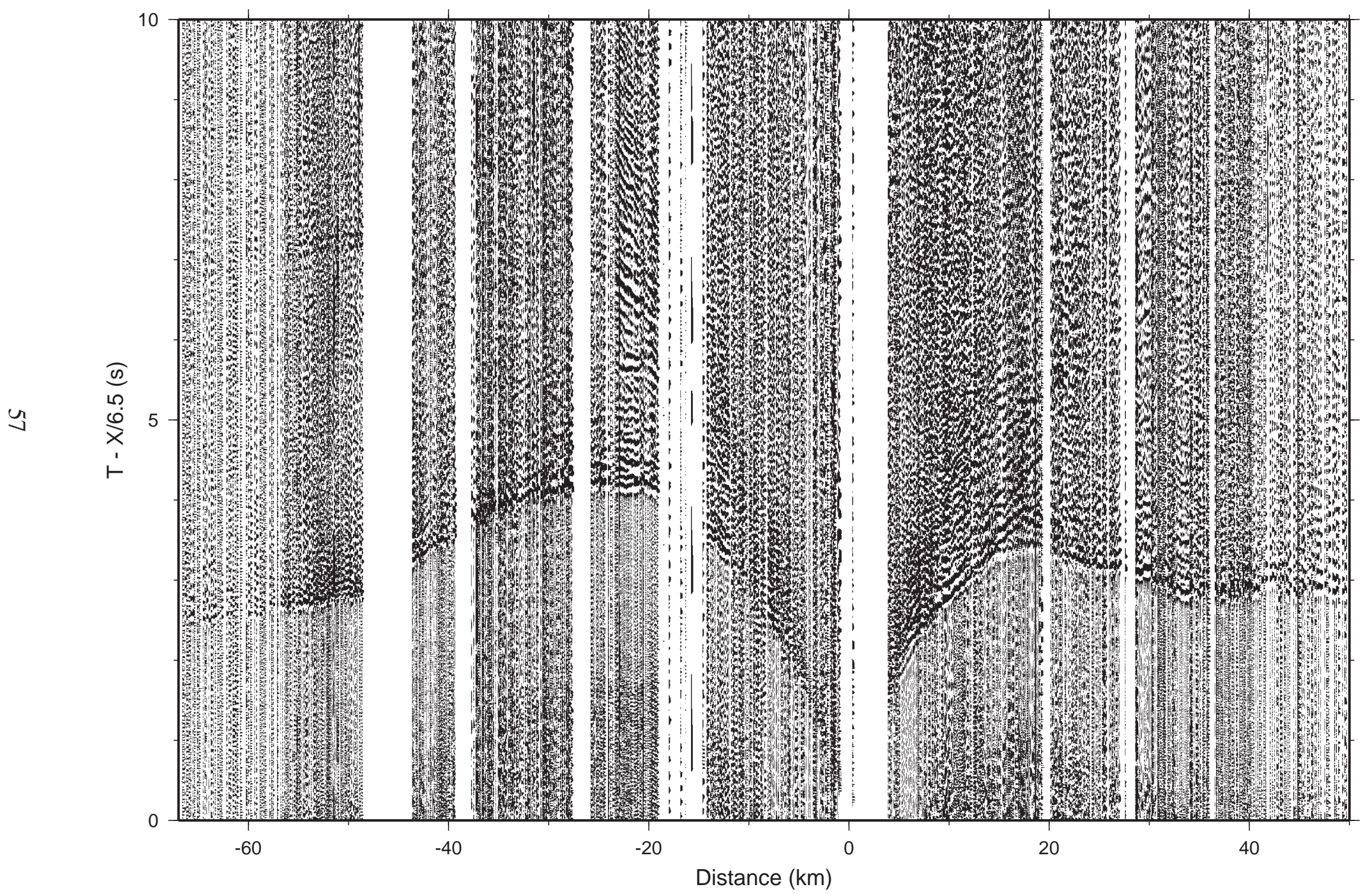

Figure 25. Reduced record section for Shotpoint 21b, vertical component only, for Lines 1 and 2. 


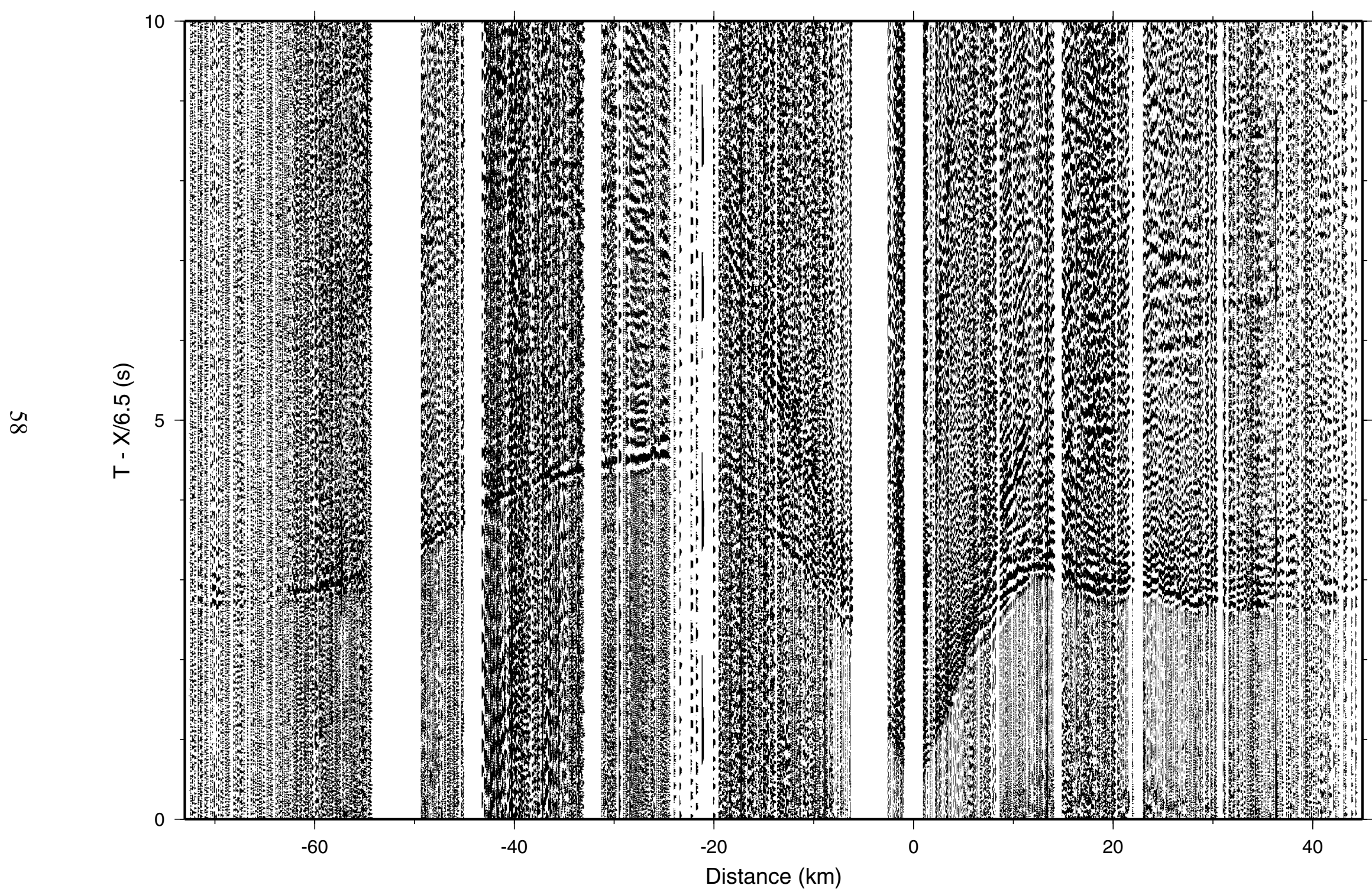

Figure 26. Reduced record section for Shotpoint 22, vertical component only, for Lines 1 and 2. 


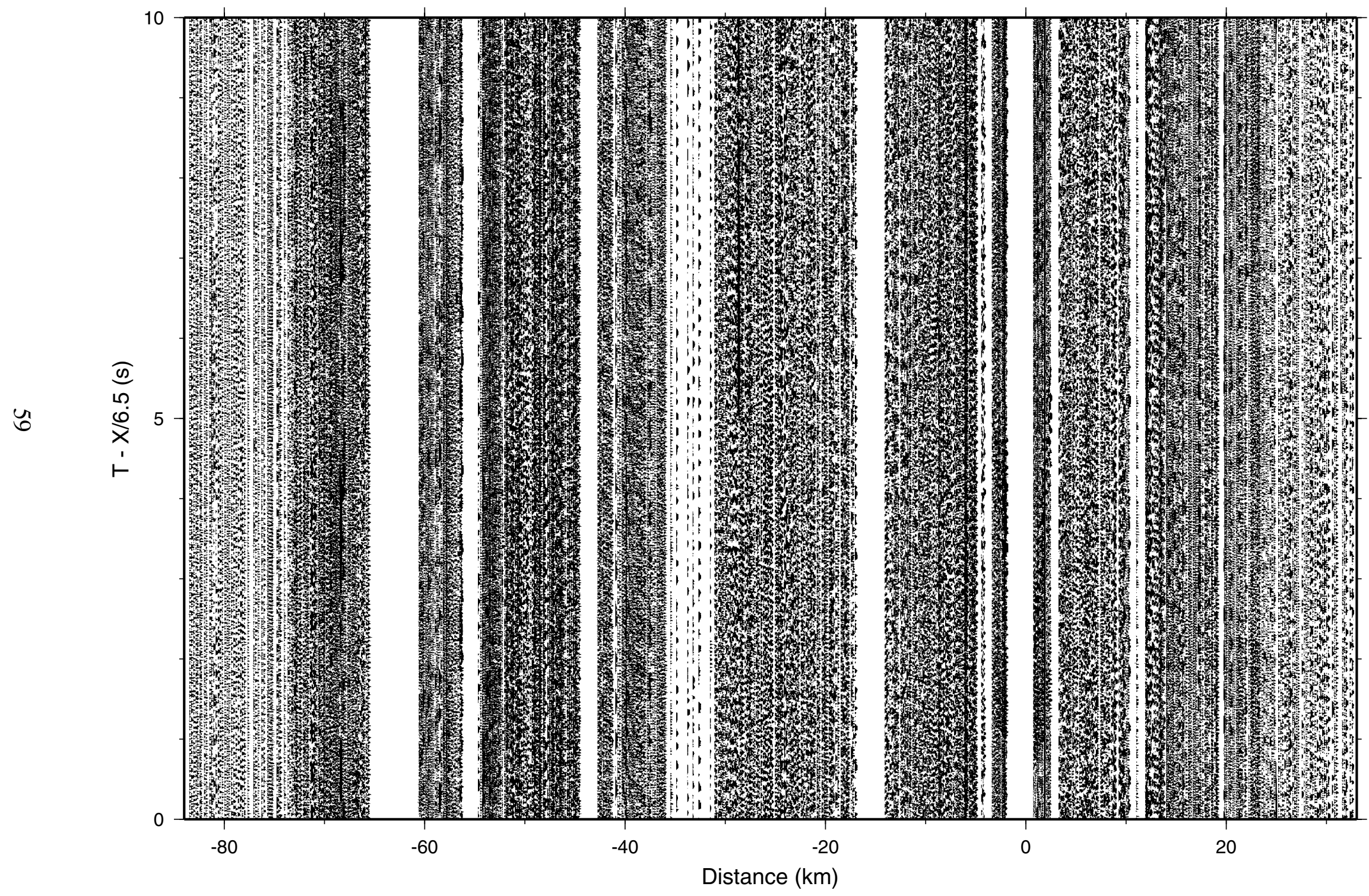

Figure 27. Reduced record section for Shotpoint 24a, vertical component only, for Lines 1 and 2. 


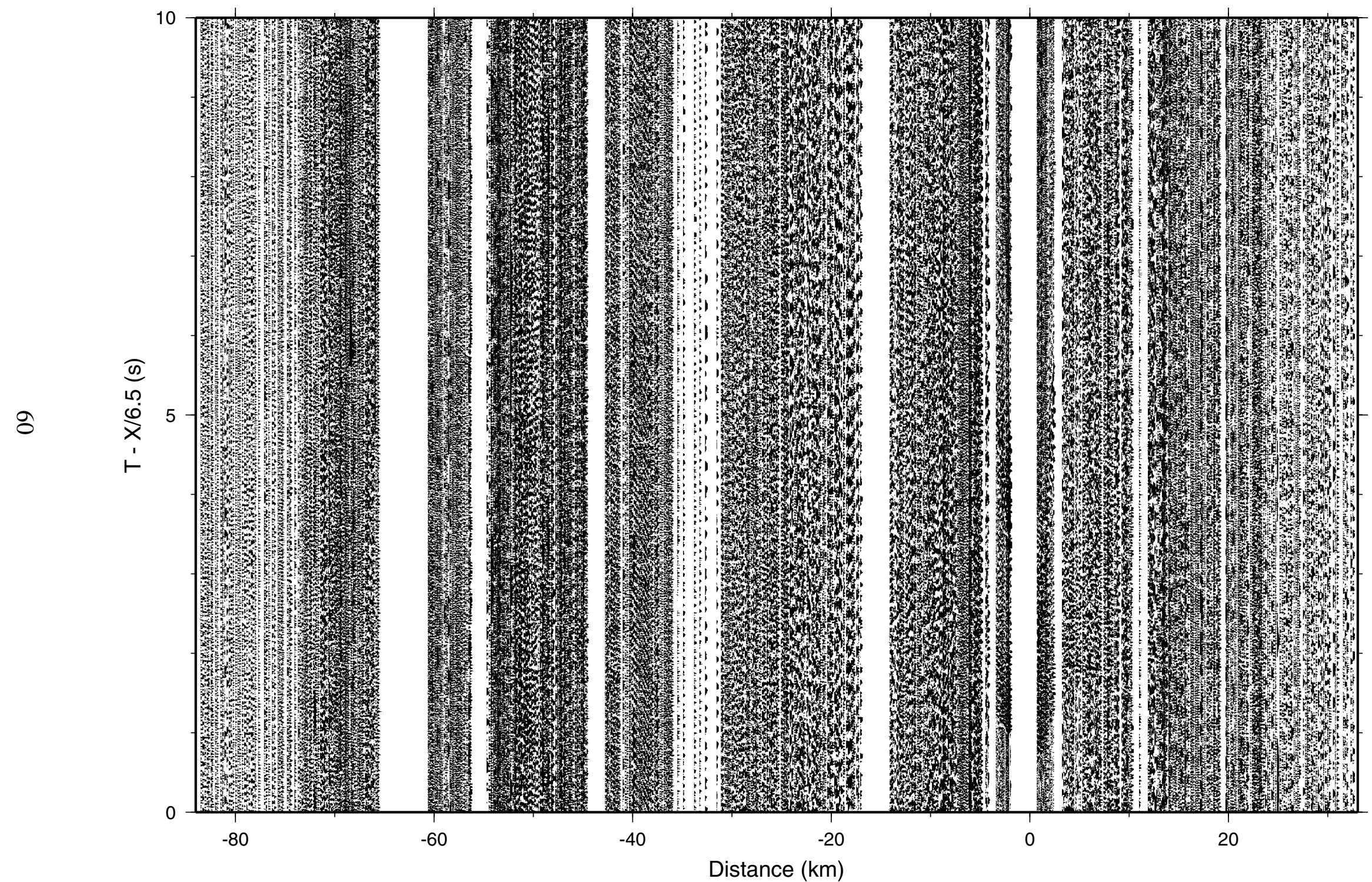

Figure 28. Reduced record section for Shotpoint 24b, vertical component only, for Lines 1 and 2. 


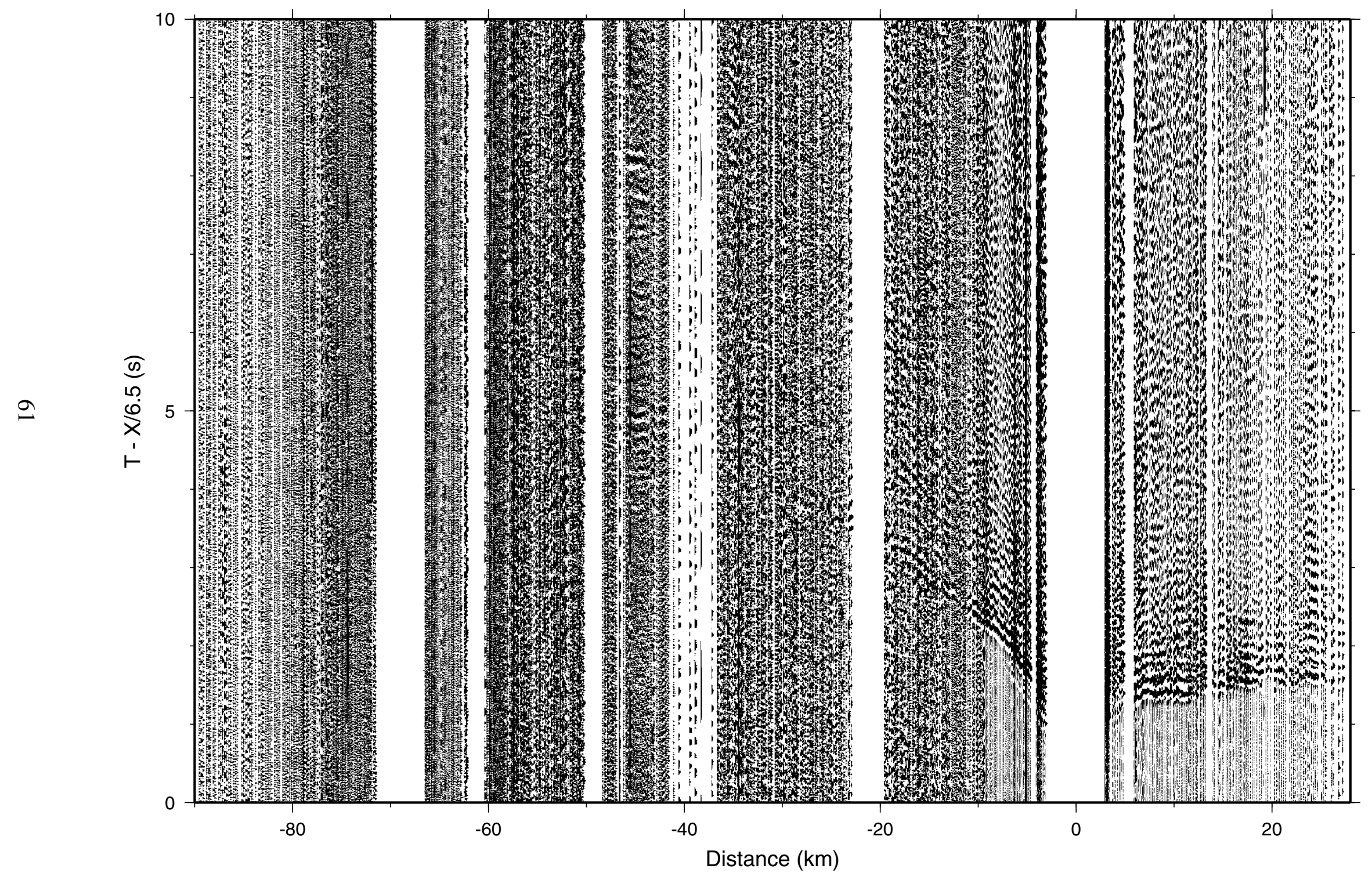

Figure 29. Reduced record section for Shotpoint 26a, vertical component only, for Lines 1 and 2. 


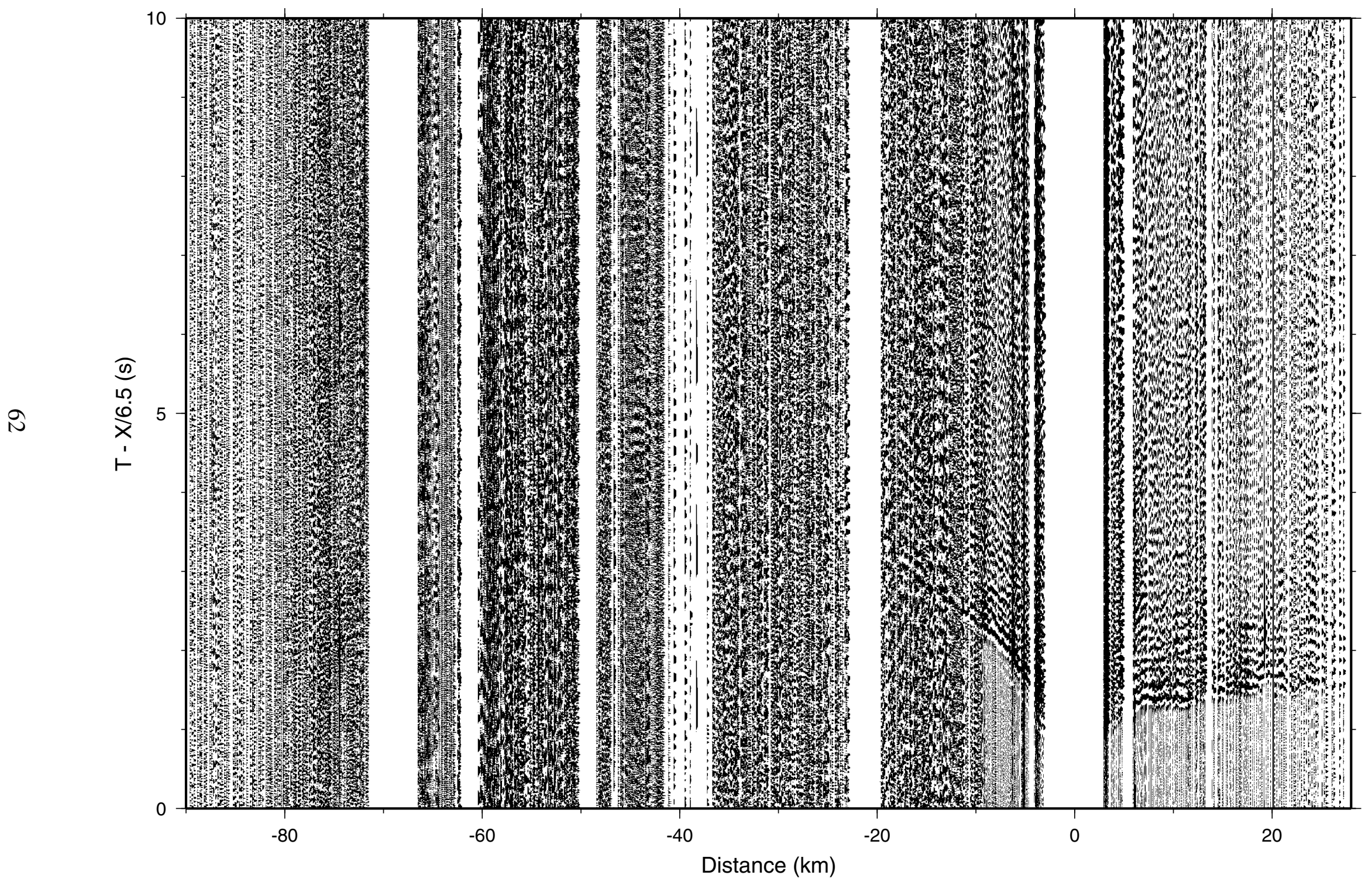

Figure 30. Reduced record section for Shotpoint 26b, vertical component only, for Lines 1 and 2. 


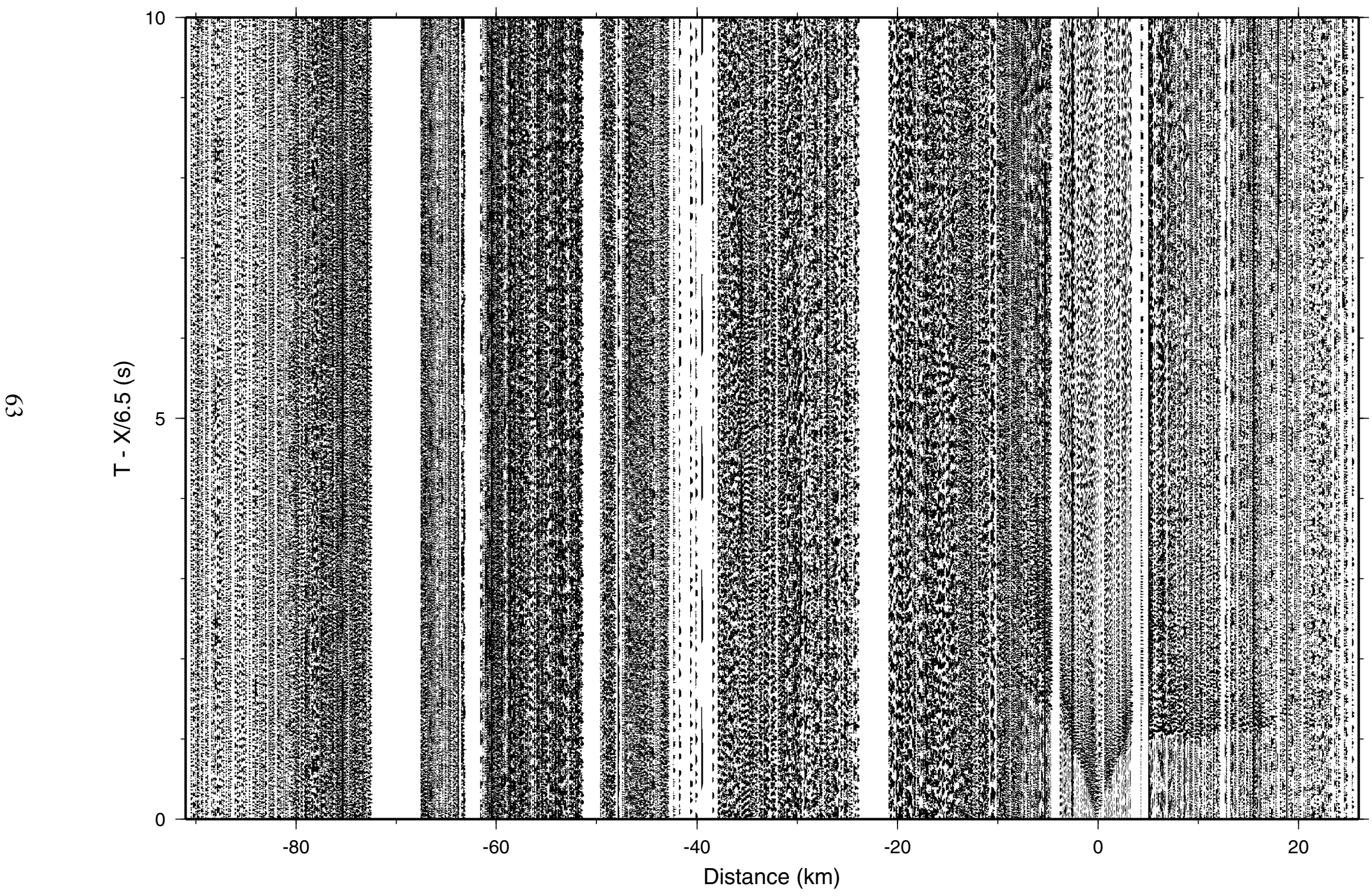

Figure 31. Reduced record section for Shotpoint 27, vertical component only, for Lines 1 and 2. 


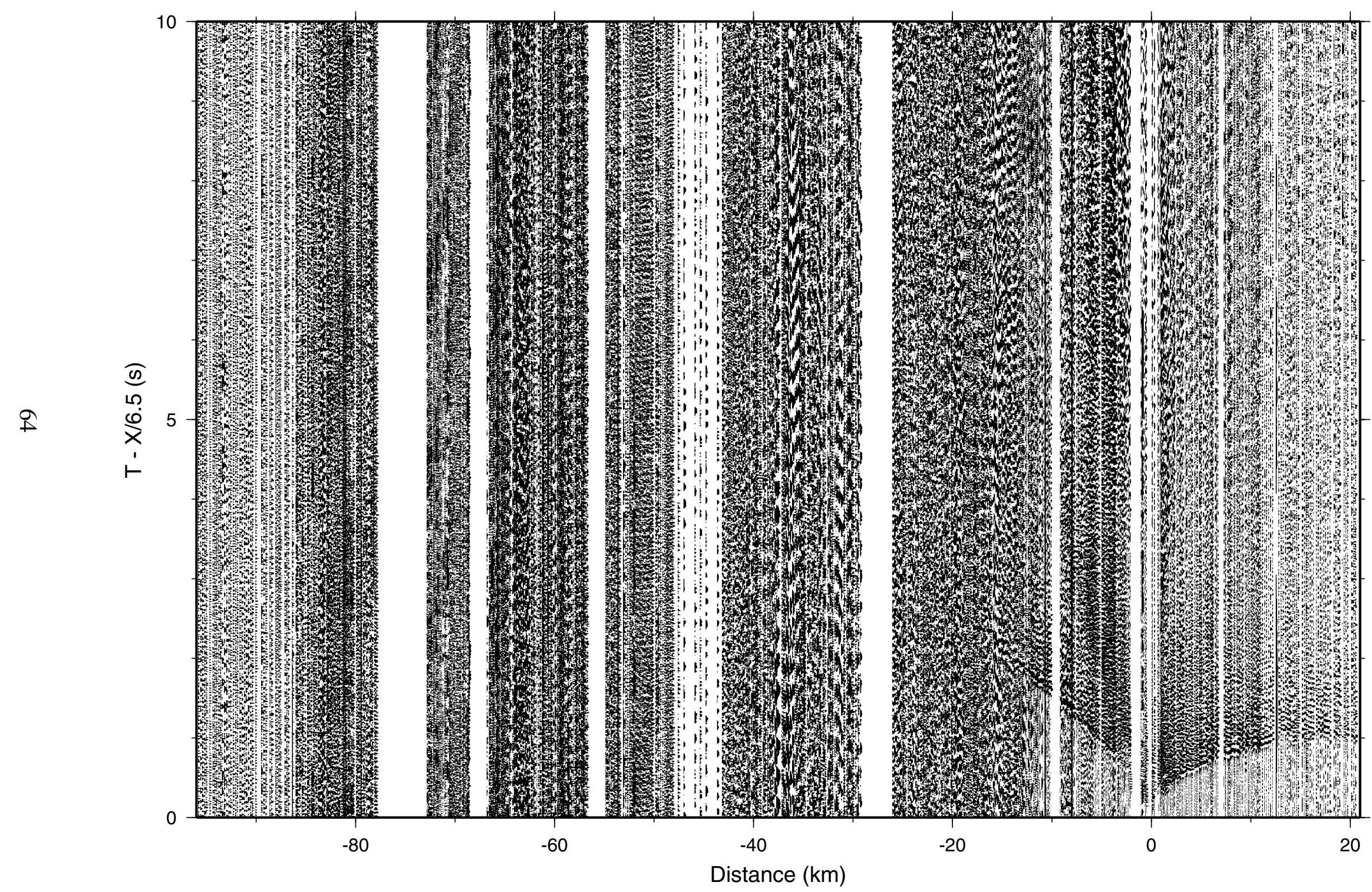

Figure 32. Reduced record section for Shotpoint 29, vertical component only, for Lines 1 and 2. 


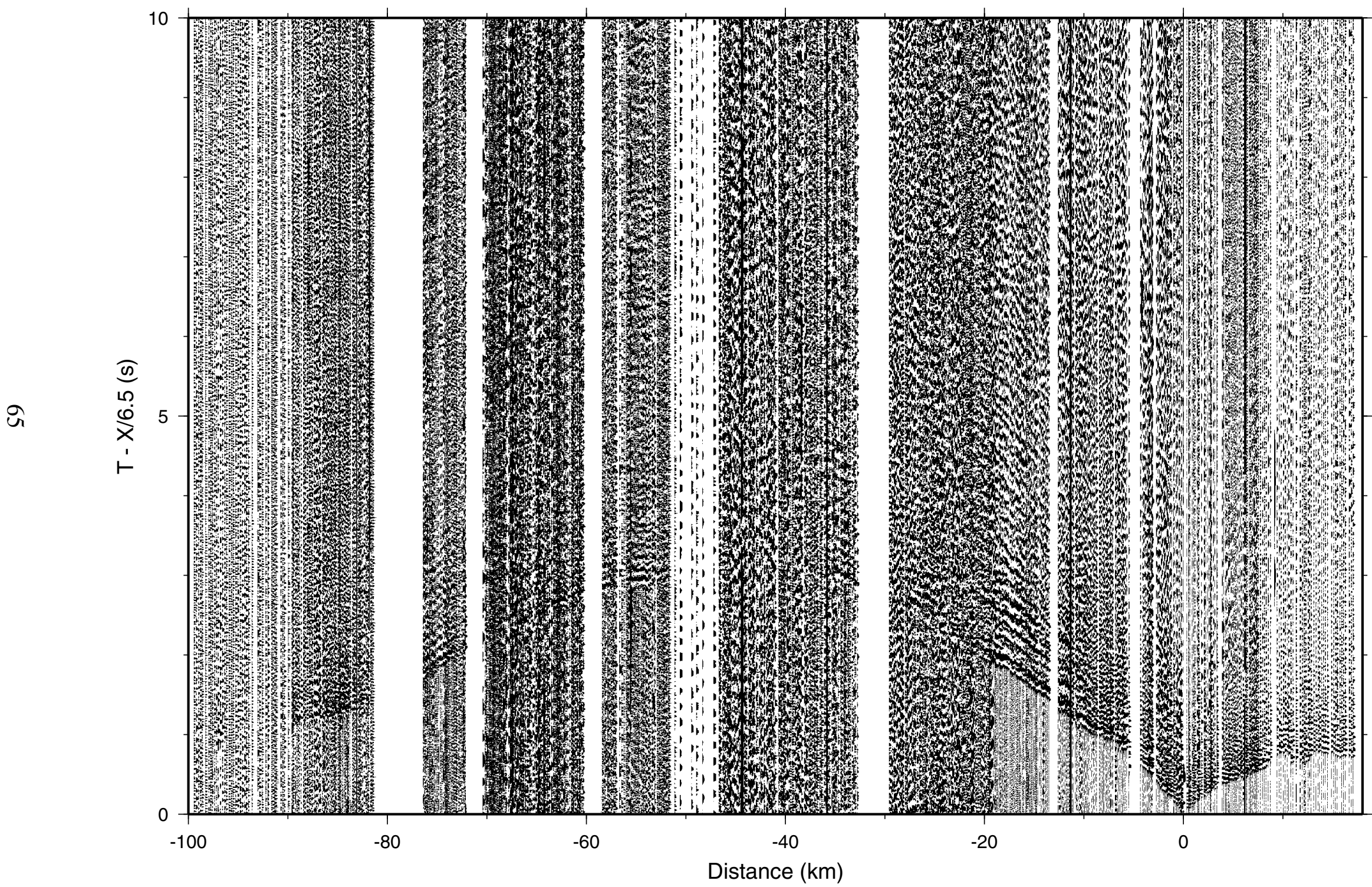

Figure 33. Reduced record section for Shotpoint 30, vertical component only, for Lines 1 and 2. 


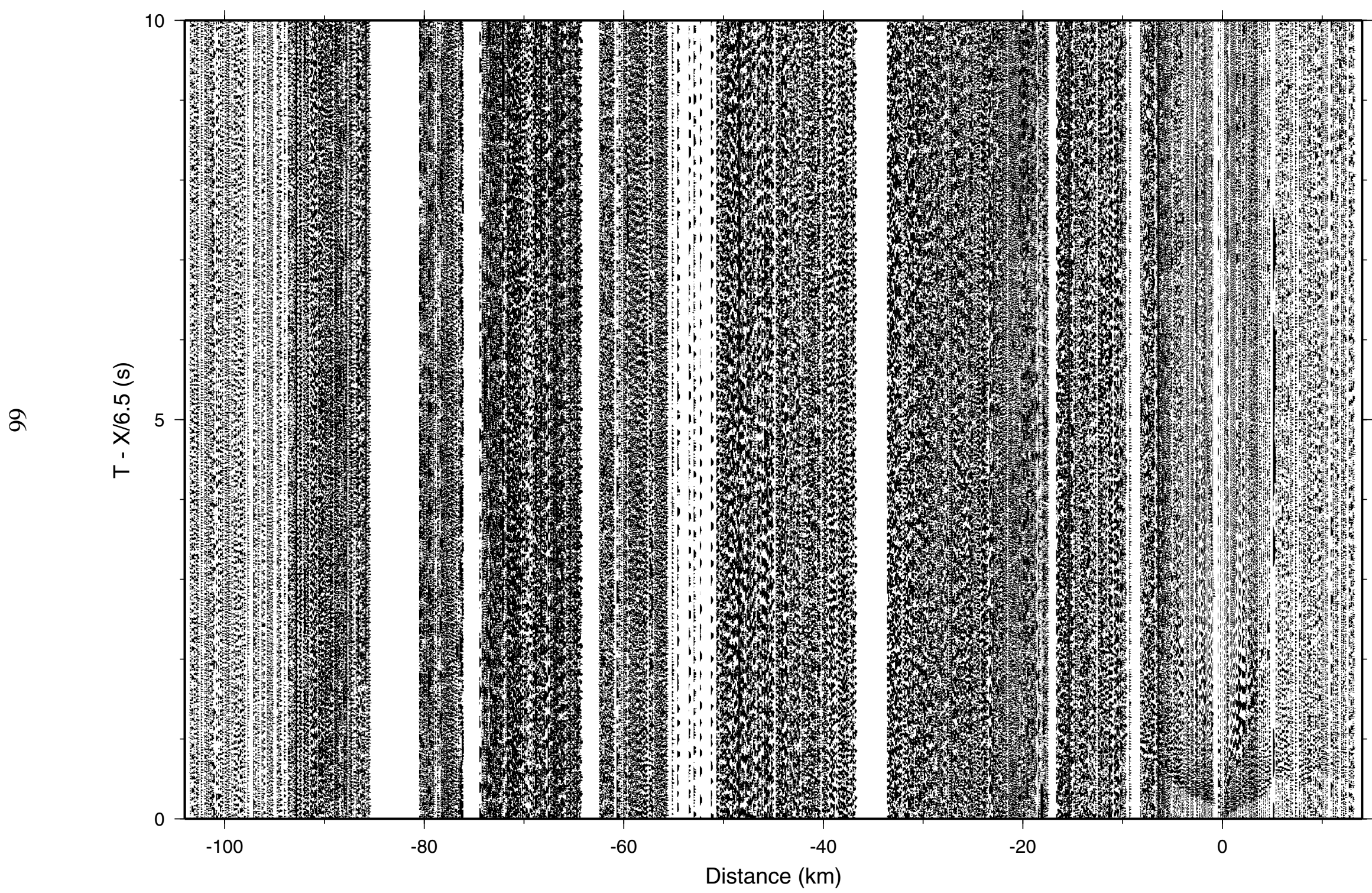

Figure 34. Reduced record section for Shotpoint 31, vertical component only, for Lines 1 and 2. 


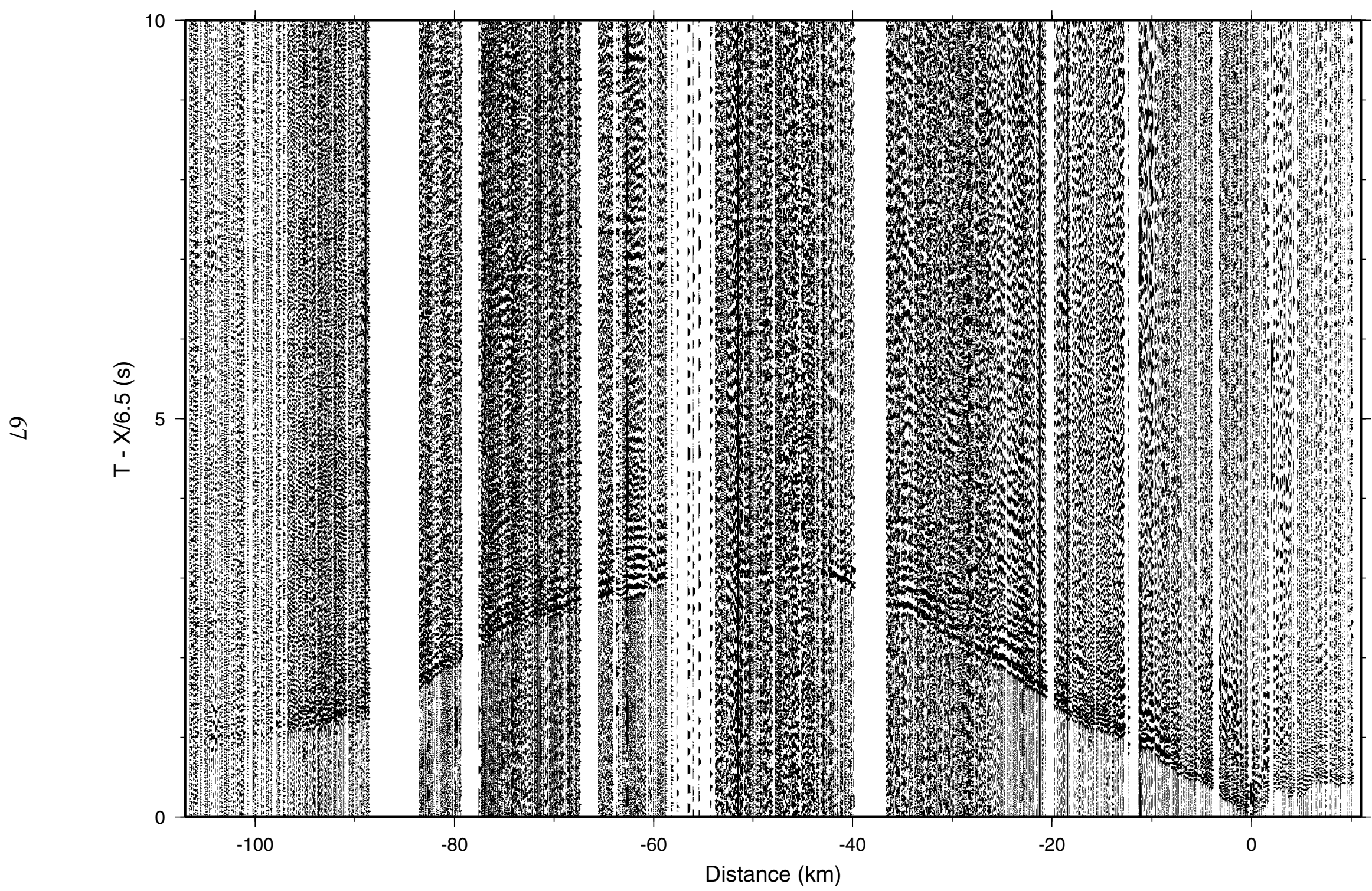

Figure 35. Reduced record section for Shotpoint 32a, vertical component only, for Lines 1 and 2. 


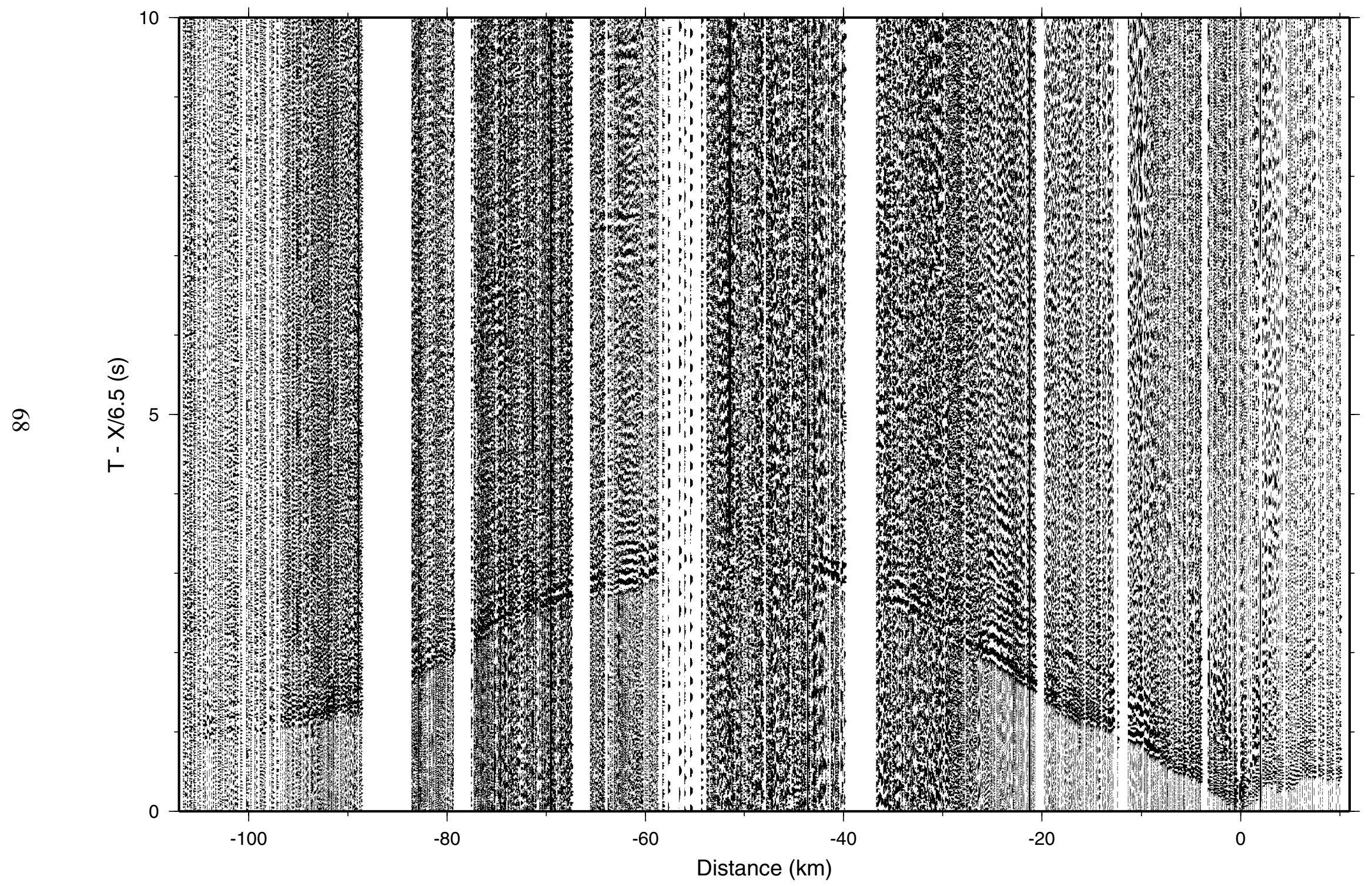

Figure 36. Reduced record section for Shotpoint 32b, vertical component only, for Lines 1 and 2. 


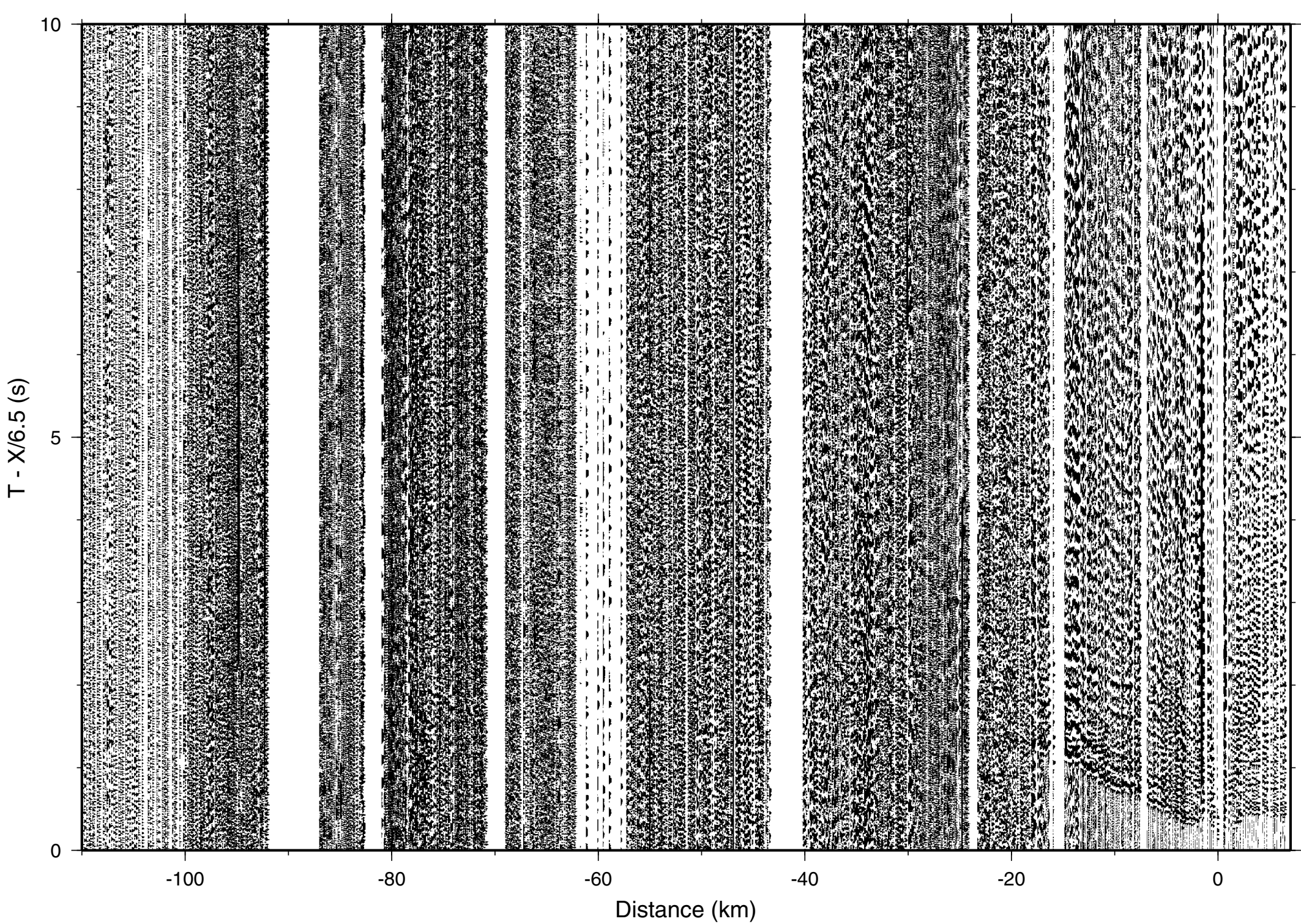

Figure 37. Reduced record section for Shotpoint 33, vertical component only, for Lines 1 and 2. 


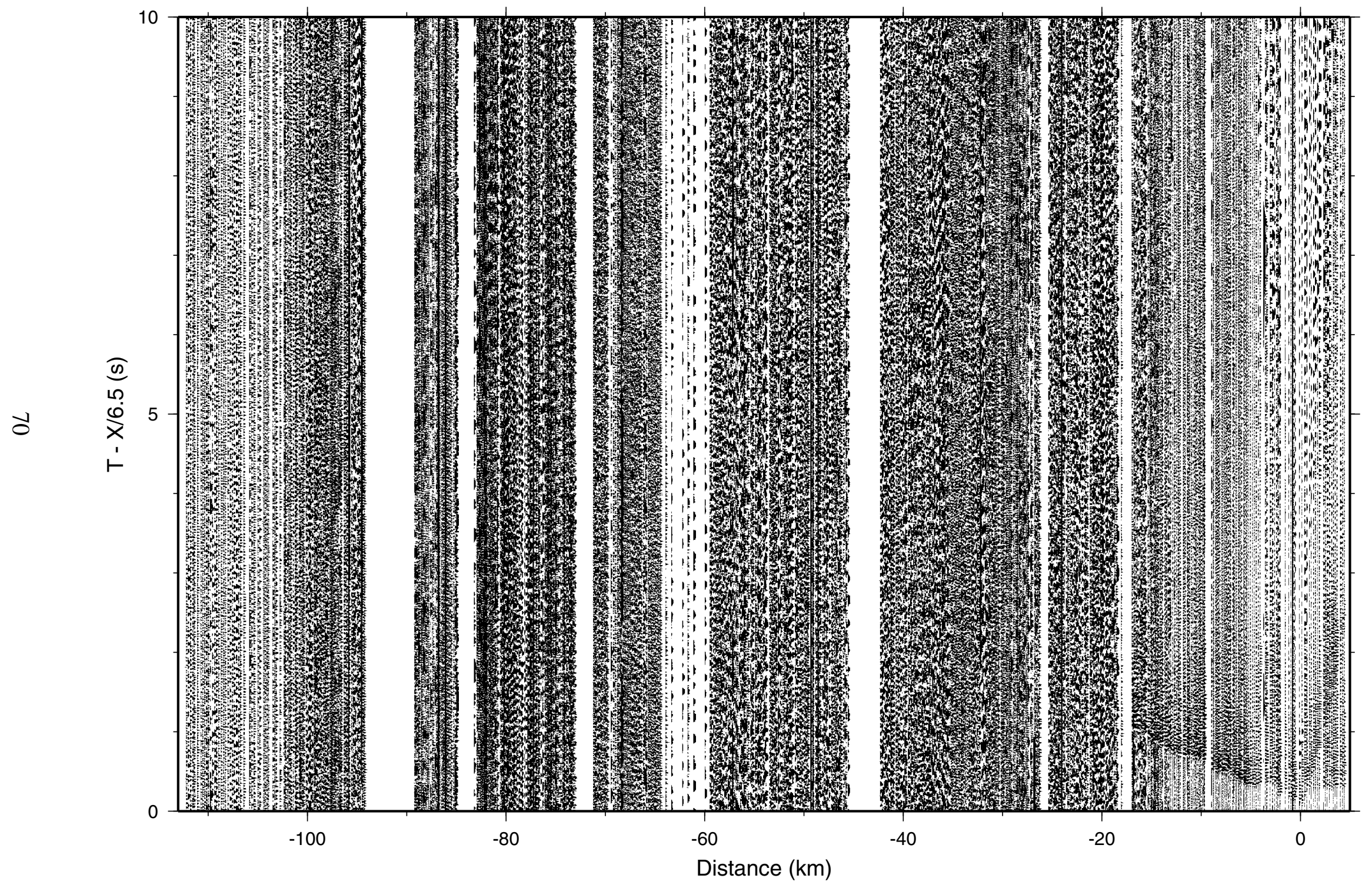

Figure 38. Reduced record section for Shotpoint 34, vertical component only, for Lines 1 and 2. 


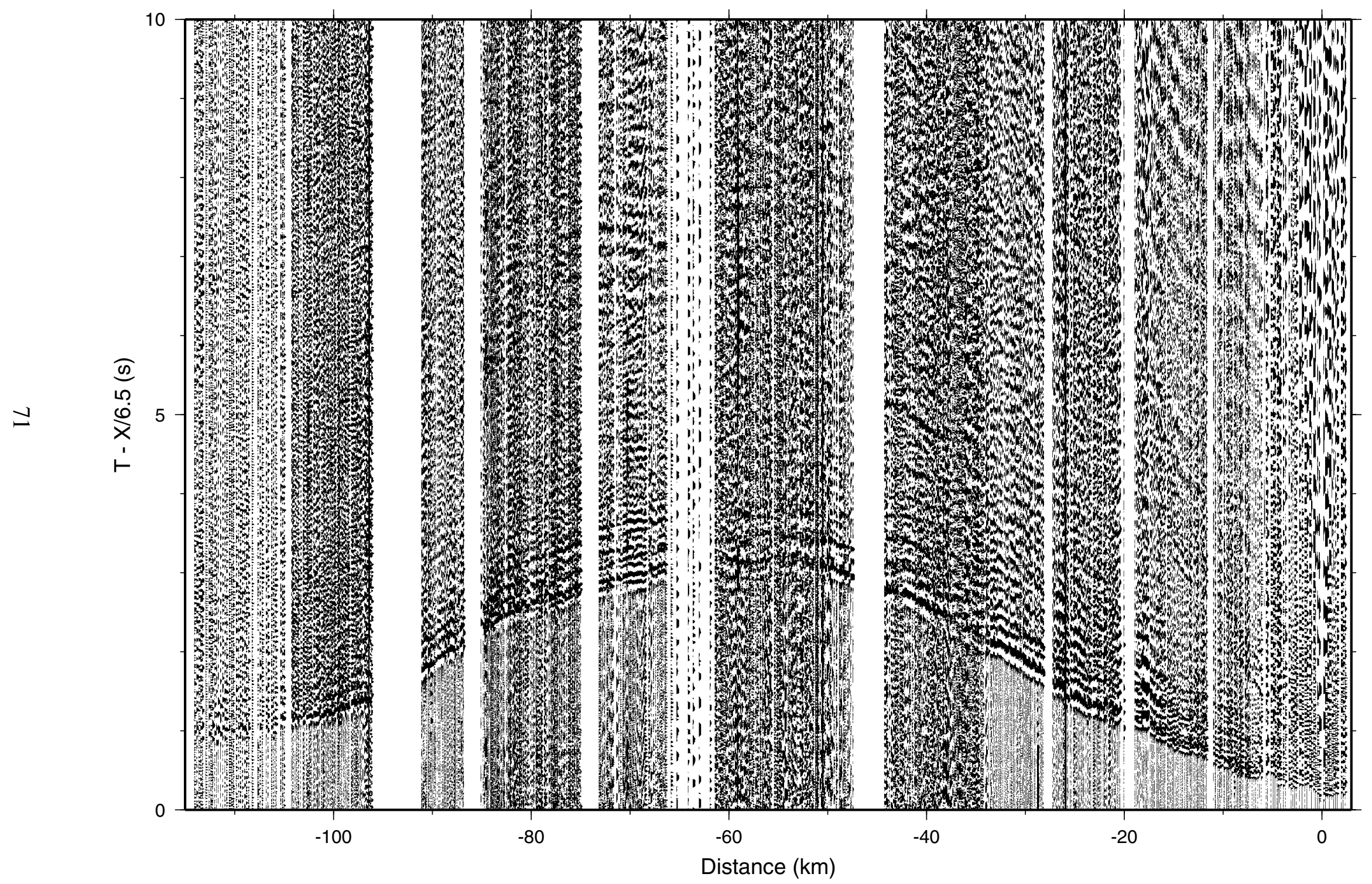

Figure 39. Reduced record section for Shotpoint 35, vertical component only, for Lines 1 and 2. 
1999/9/20 11:16:54 47.6N 121.8W 17km 0.0 BHZ

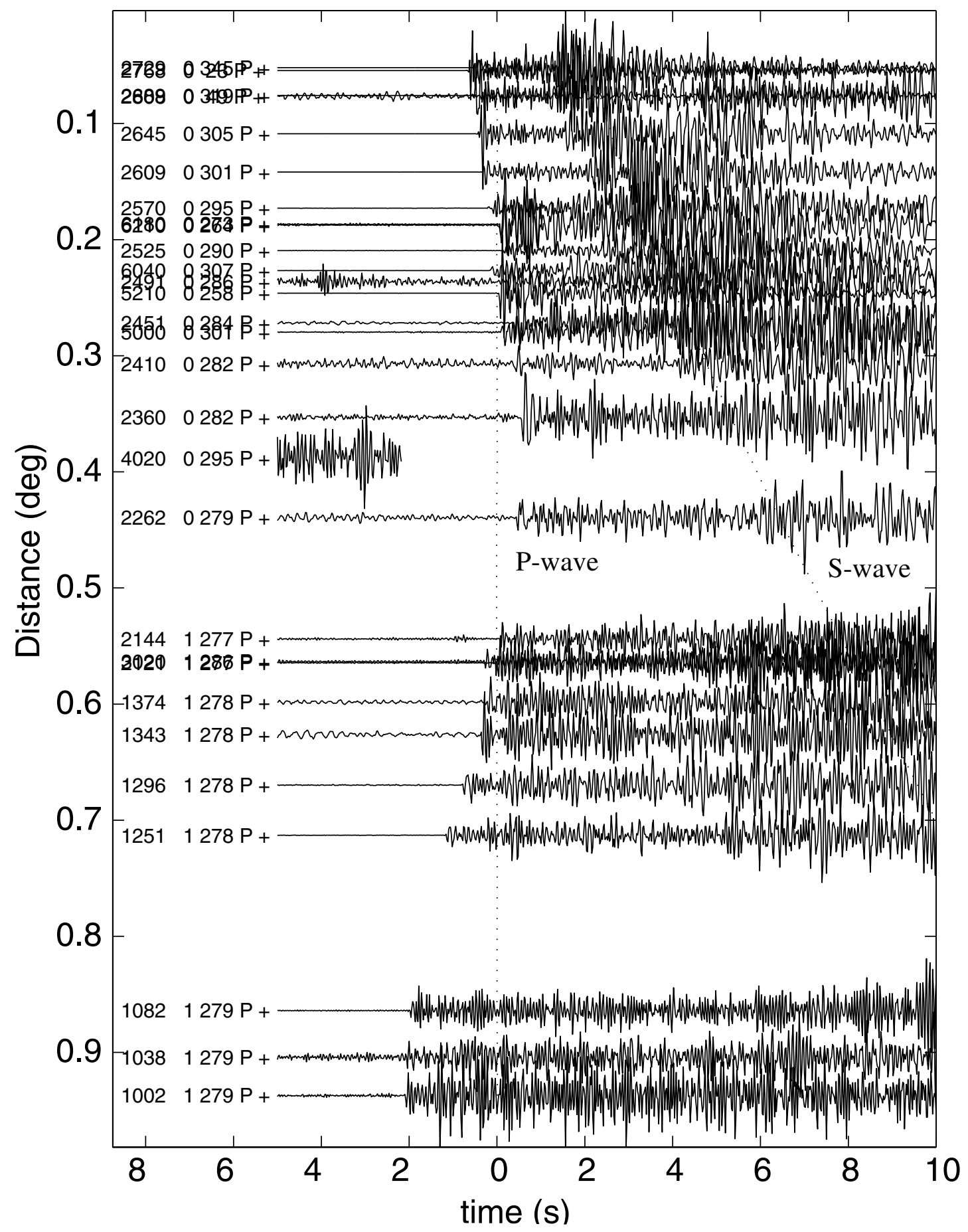

Figure 40. Vertical component REFTEK recordings of a local M2.8 earthquake on 9/20/1999 at $17 \mathrm{~km}$ depth (event 11 on Table 6a). Numbers on left side of traces show station numbers of the receivers; numbers between 279 and 305 show azimuth between the earthquake and the receiver. Dotted lines show locations of P- and S-wave arrivals calculated from the iasp91 earth model. 


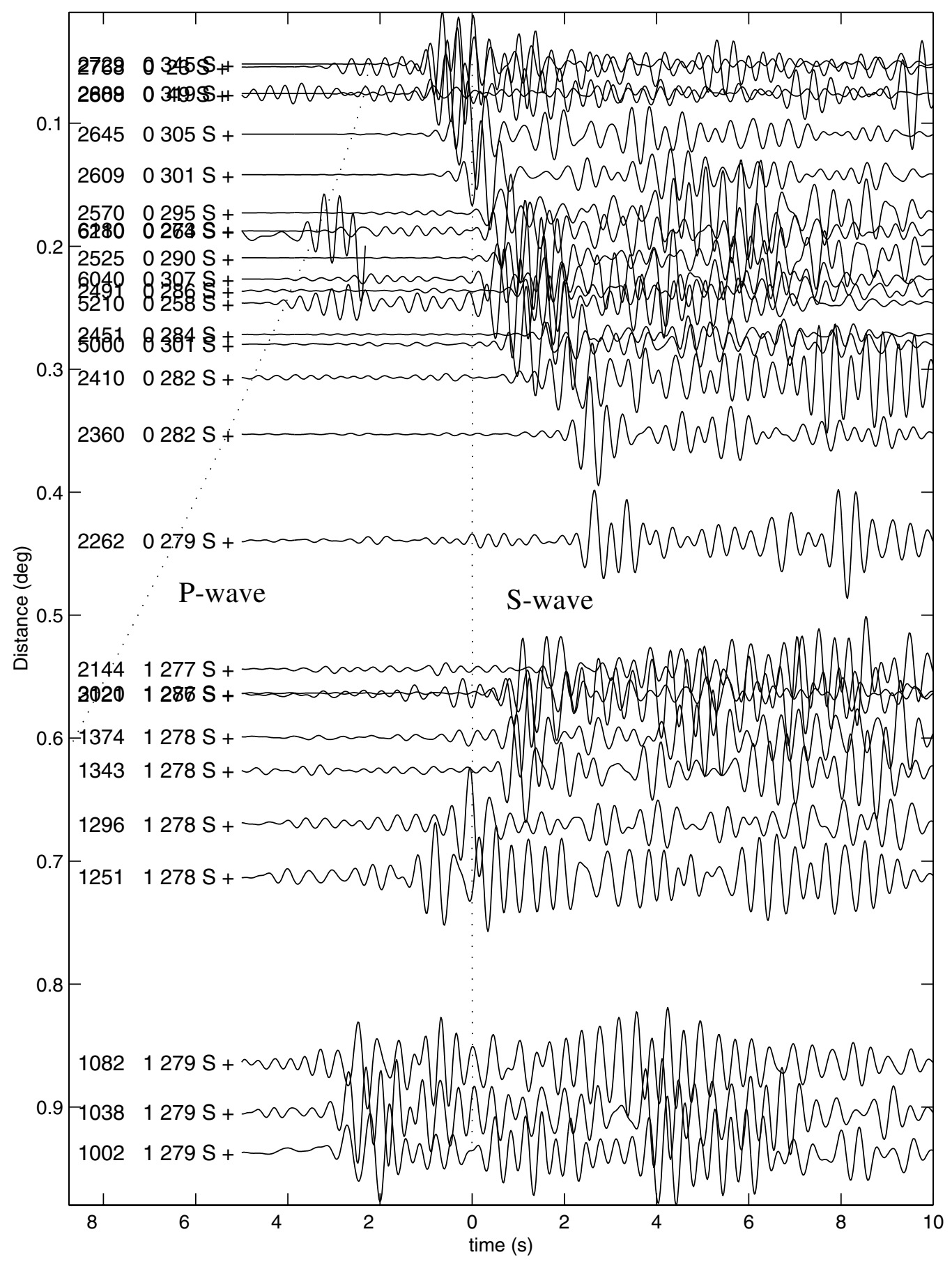

Figure 41. Horizontal component (N-S) REFTEK recordings of a local M2.8 earthquake on 9/20/1999 at $17 \mathrm{~km}$ depth (event 11 on Table 6a). Numbers on left side of traces show station numbers of the receivers; numbers between 279 and 305 show azimuth between the earthquake and the receiver. Dotted lines show locations of P- and S-wave arrivals calculated from the iasp91 earth model. 


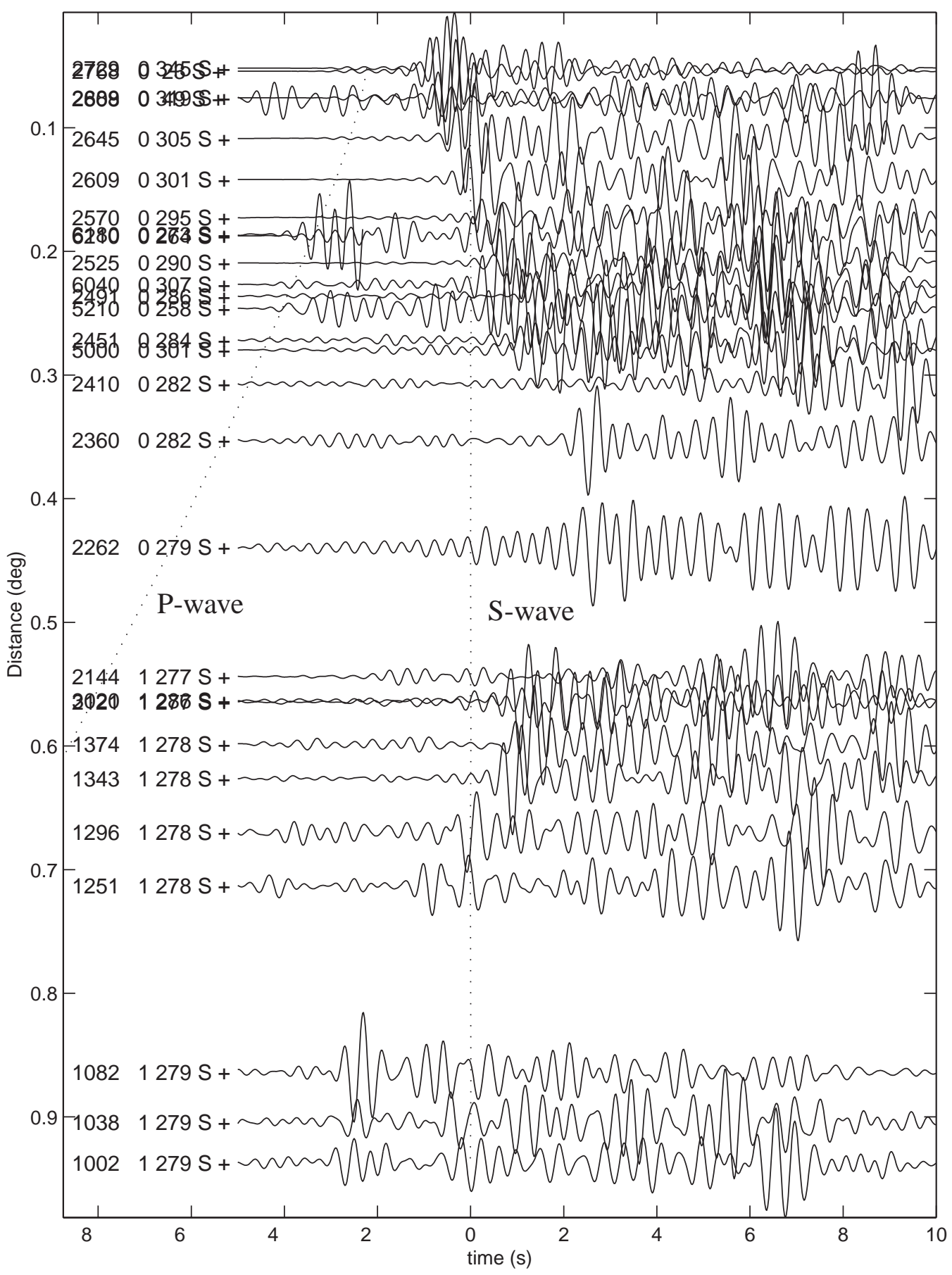

Figure 42. Horizontal component (E-W) REFTEK recordings of a local M2.8 earthquake on 9/20/1999 at $17 \mathrm{~km}$ depth (event 11 on Table 6a). Numbers on left side of traces show station numbers of the receivers; numbers between 279 and 305 show azimuth between the earthquake and the receiver. Dotted lines show locations of P- and S-wave arrivals calculated from the iasp91 earth model. 


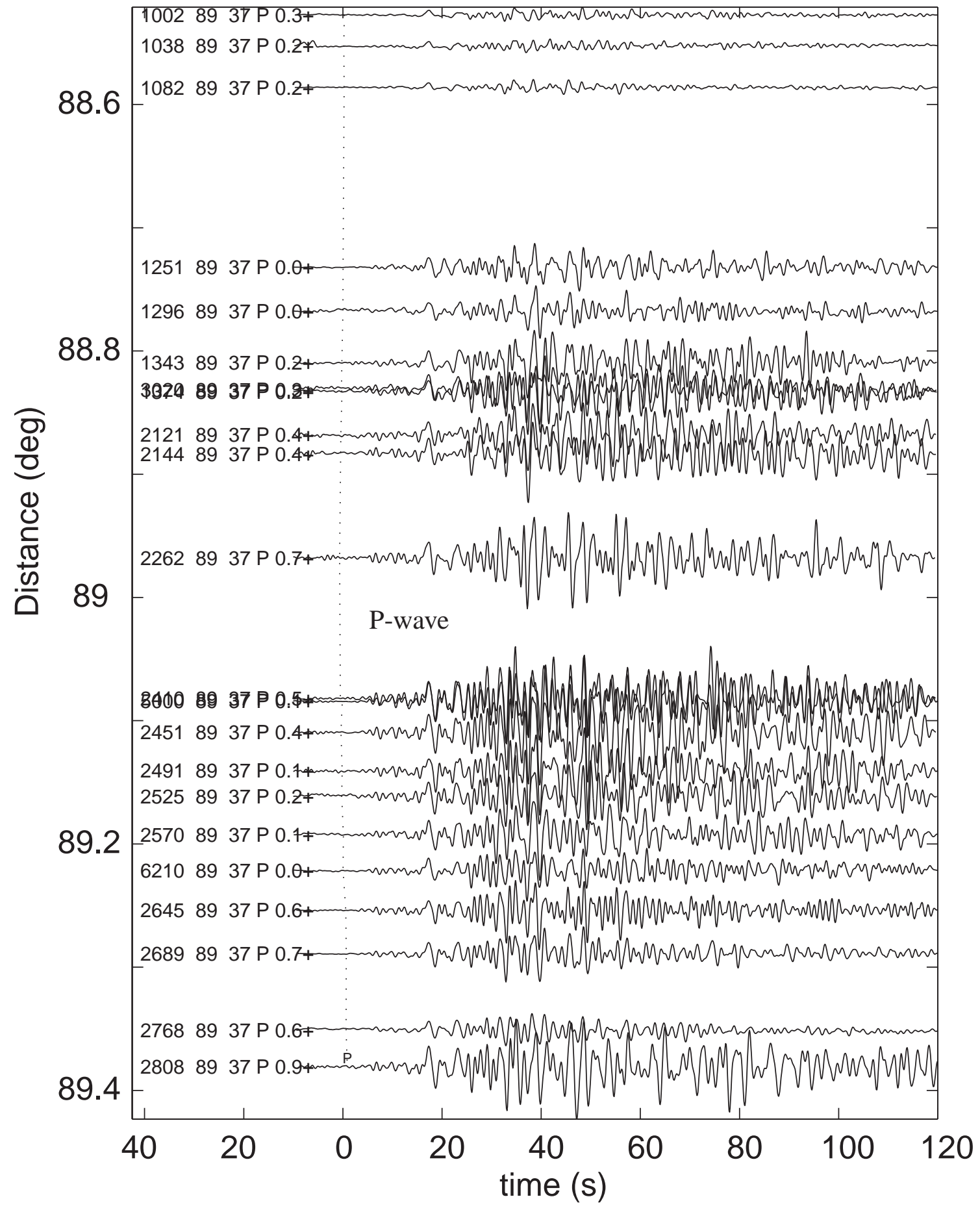

Figure 43. Vertical component REFTEK recordings of the M7.6 Chi Chi earthquake on 9/20/1999. Numbers on left side of traces show station numbers of the receivers; numbers between 0.0 and $0.9+$ show shift applied to the trace. Dotted line shows locations of P-wave arrival calculated from the iasp91 earth model. 


\section{P-wave}

88.7

D্d
$\frac{0}{0}$
0
0
$\frac{\pi}{\pi}$
$\frac{\pi}{0}$
$\frac{0}{0}$

$12518937 \mathrm{P} 0.0+: \mathrm{N}$ : N $12968937 \mathrm{P} 0.0+\mathrm{N}$ $8 8 . 8 \longdiv { 1 3 4 3 \quad 8 9 } 3 7$ P $0.2+$

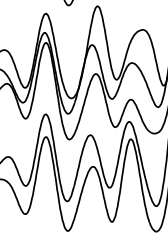

$30208937 P 0 . \%$ $88.9-$

$21218937 \times 0.4+$ 21448937 P 0.47

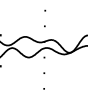




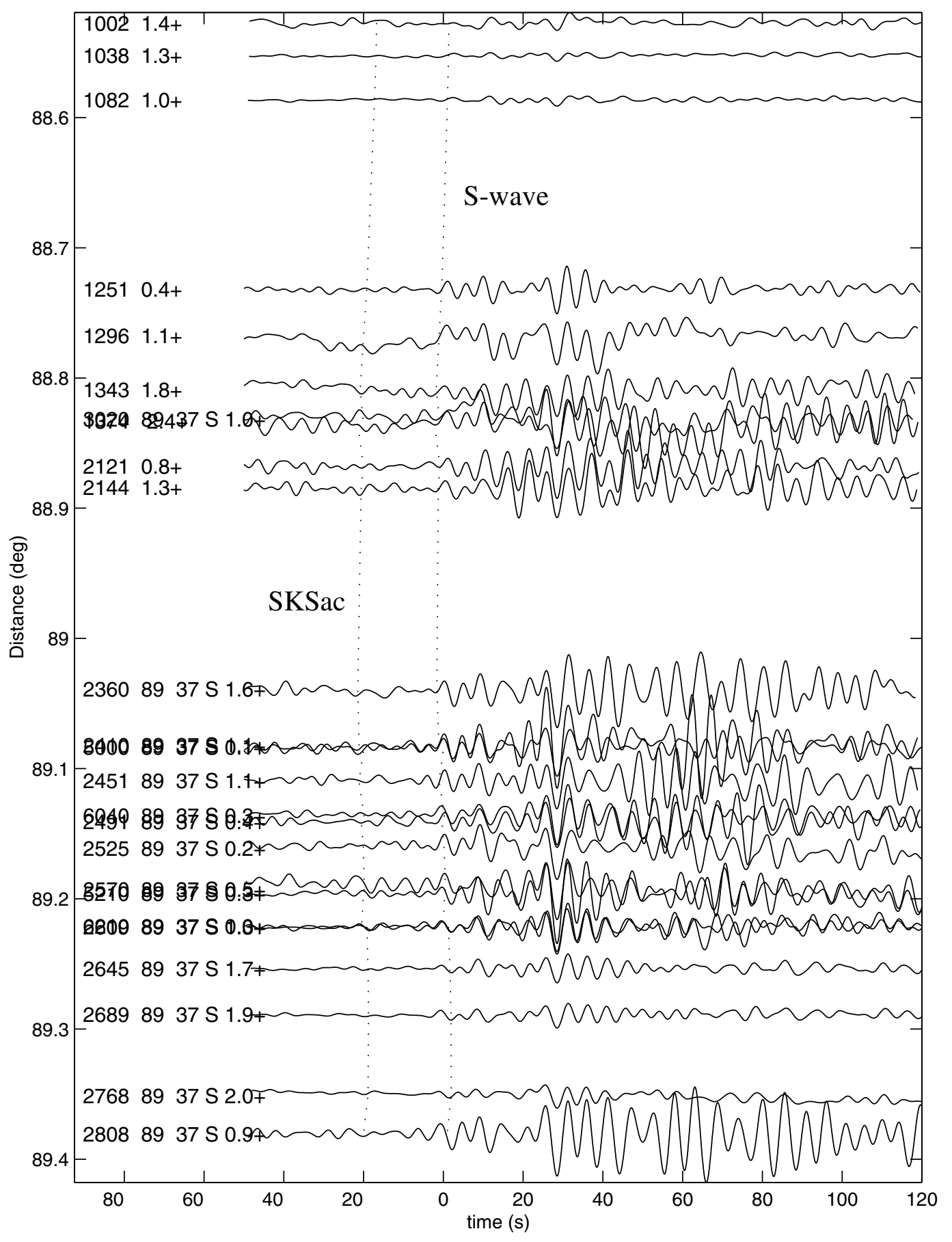

Figure 45. East-west horizontal component REFTEK recordings of the M7.6 Chi Chi earthquake on 9/20/1999. Numbers on left side of traces show station numbers of the receivers; numbers between 0.0 and 2.0+ show shift applied to the trace. Dotted line shows locations of SKS and S-wave arrivals calculated from the iasp91 earth model. Data have been low pass filtered with an upper corner at $0.25 \mathrm{~Hz}$. 


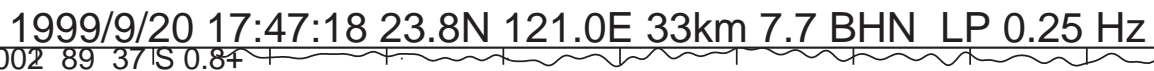

10388937 S $2.7+$

88.6

10828937 S $0.8+$

\section{S-wave}

$88.7-$

$1251893750.7+$ $1296893750.1+$ 88.8

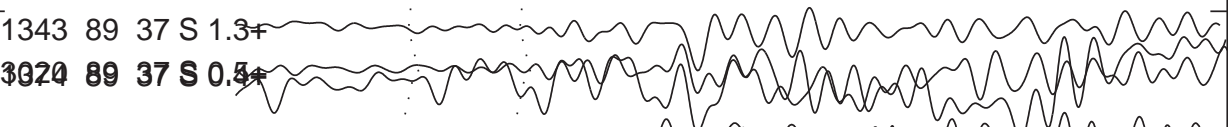
21218937 S2.74 21448937 S.74)

SKSac

$89-$

89.1

23608937 s $0.7+m$ mann

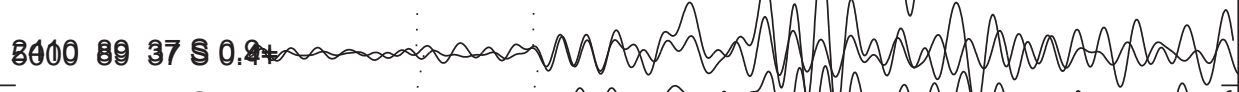
$24518937 \mathrm{S1.2*}$ 2499893780.39 $25258937 \mathrm{~S} 0.2 \mathrm{~m}$

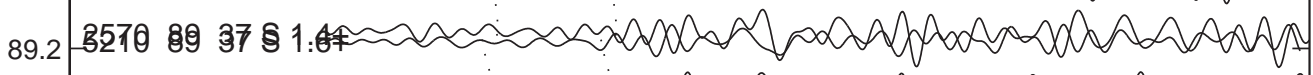
$88008937 \mathrm{~S} 8.8+20000000000000000$

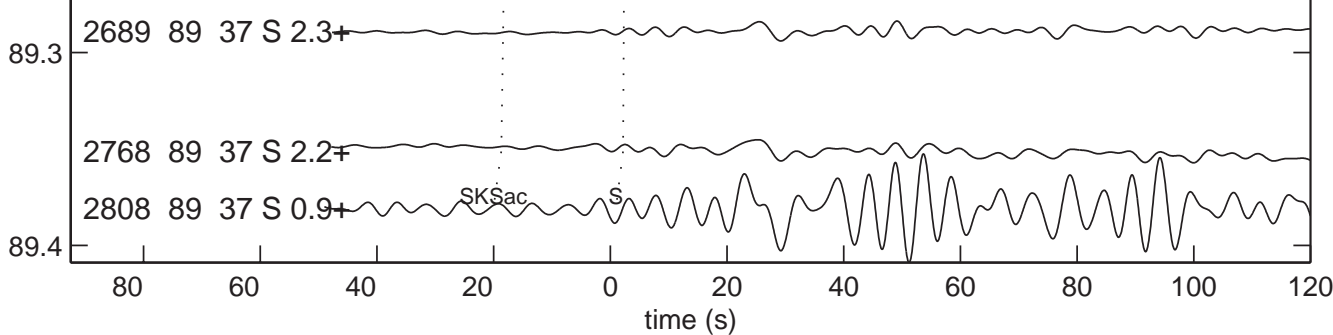

Figure 46. North-south horizontal component REFTEK recordings of the M7.6 Chi Chi earthquake on $9 / 20 / 1999$. Numbers on left side of traces show station numbers of the receivers; numbers between 0.0 and 2.7+ show shift applied to the trace. Dotted line shows locations of SKS and S-wave arrivals calculated from the iasp91 earth model. Data have been low pass filtered with an upper corner at $0.25 \mathrm{~Hz}$. 
1999/9/20 17:47:18 23.8N 121.0E 33km 7.7 BHN LP 0.1 Hz

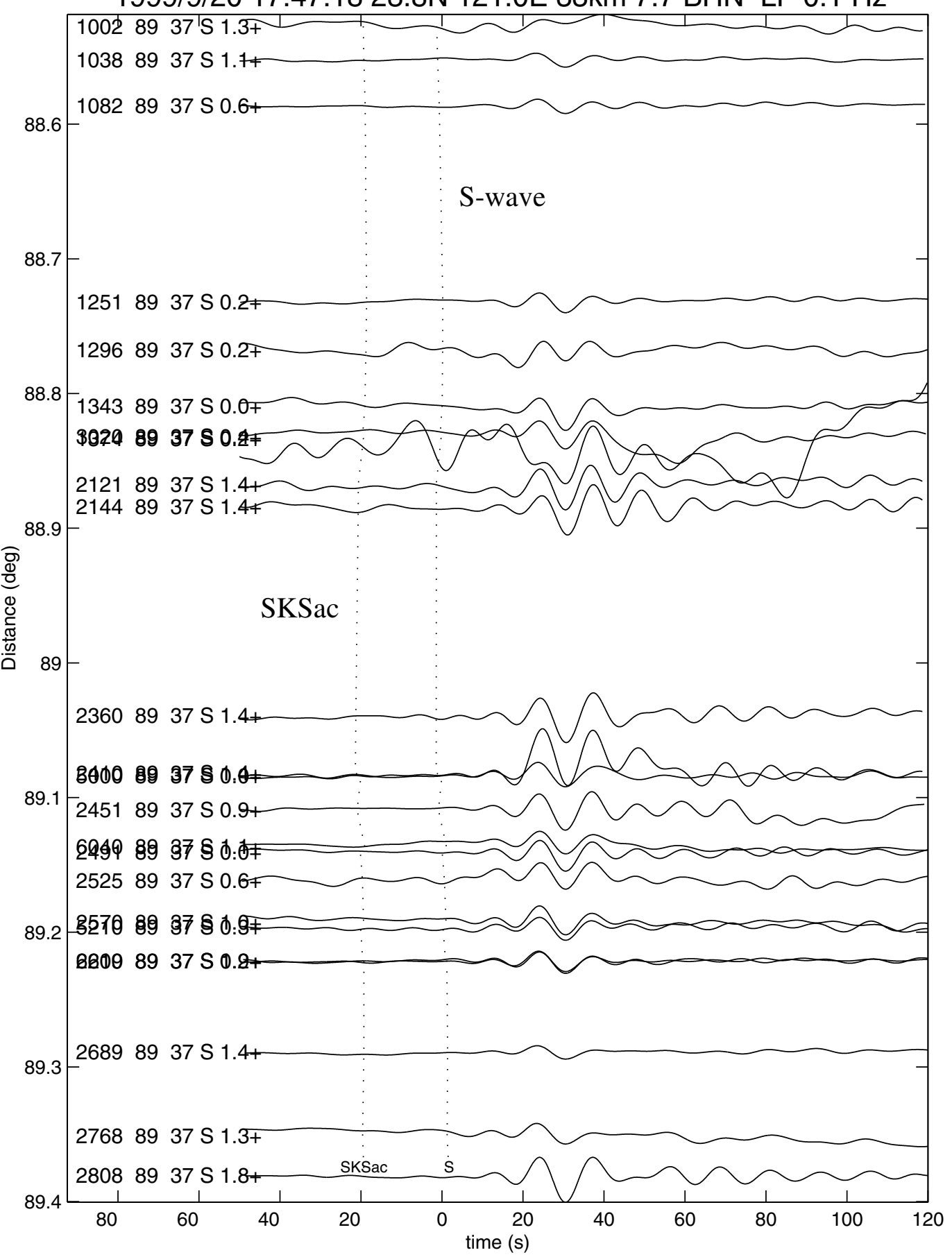

Figure 47. North-south horizontal component REFTEK recordings of the M7.6 Chi Chi earthquake on 9/20/1999. Numbers on left side of traces show station numbers of the receivers; numbers between 0.0 and $1.8+$ show shift applied to the trace. Dotted line shows locations of SKS and S-wave arrivals calculated from the iasp91 earth model. Data have been low pass filtered with an upper corner at $0.1 \mathrm{~Hz}$. 
DRY SHIPS REFTEKs recording Taiwan Main Shock

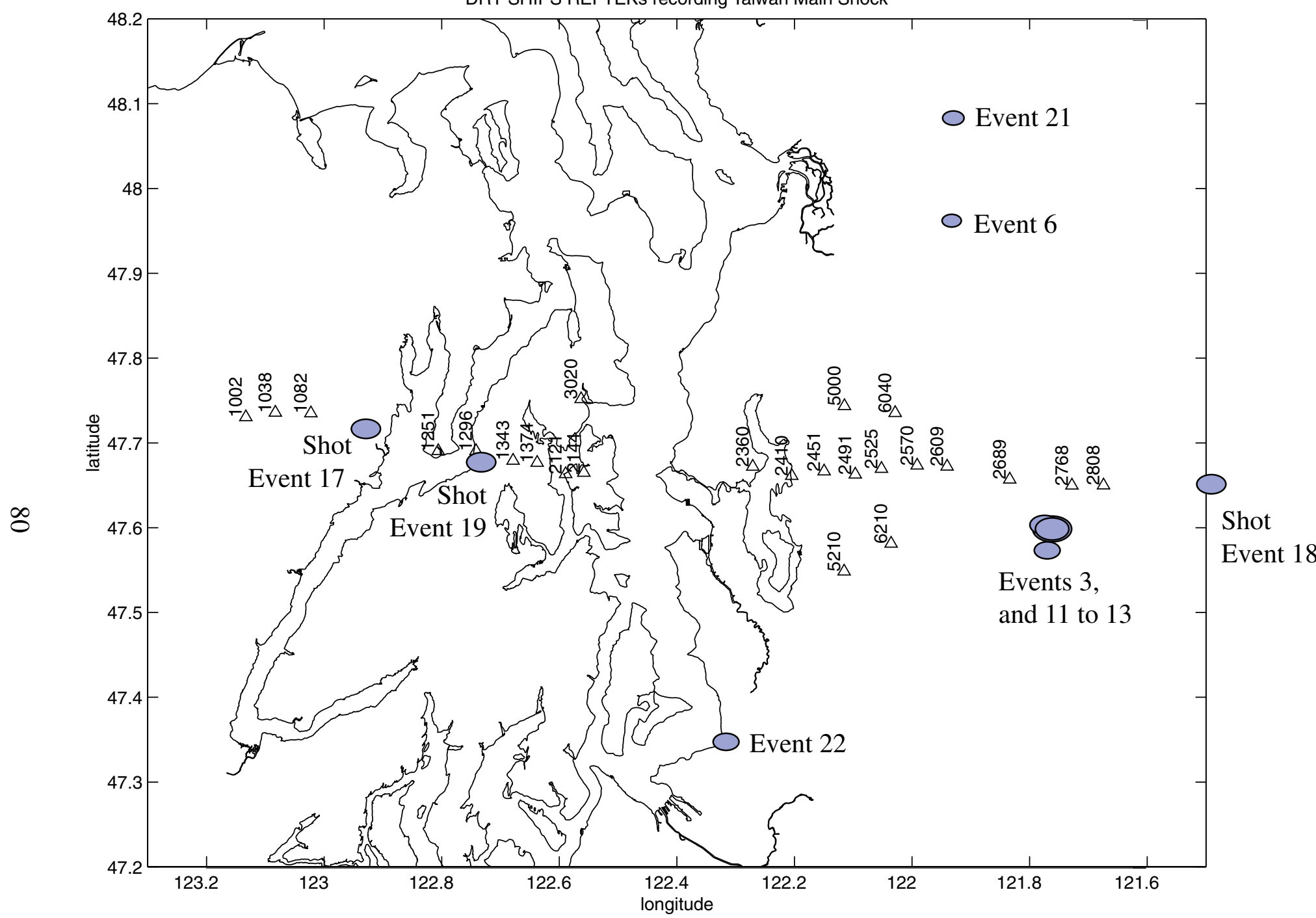

Figure 48. Map showing locations of Refteks that recorded the M7.6 Chi Chi mainshock. Ellipses show locations of local earthquakes that were also recorded by these Refteks (see Table 6a). Events 17 to 19 correspond to our own shots. 
